\title{
Sobre Folheações Finslerianas Singulares
}

\author{
Benigno Oliveira Alves
}

TESE APRESENTADA

$\mathrm{AO}$

Instituto DE MATEMÁticA E EstatísticA

DA

Universidade DE SÃo Paulo

PARA

OBTENÇÃ̃O DO TÍTULO

$\mathrm{DE}$

Doutor EM CIÊNCIAS

\author{
Programa: Matemática Pura-IME \\ Orientador: Prof. Dr. Marcos M. Alexandrino da Silva \\ Coorientador: Prof. Dr. Miguel Angel Javaloyes Victoria
}

Durante o desenvolvimento deste trabalho o autor recebeu auxílio financeiro da CNPq e PDSE-CAPES

São Paulo, Novembro de 2017 


\section{Sobre Folheações Finslerianas Singulares}

Esta versão da tese contém as correções e alterações sugeridas pela Comissão Julgadora durante a defesa da versão original do trabalho, realizada em 13/11/2017. Uma cópia da versão original está disponível no Instituto de Matemática e Estatística da Universidade de São Paulo.

Comissão Julgadora:

- Prof. Dr. Marcos Martins Alexandrino da Silva (orientador) - IME-USP

- Prof. Dr. Miguel Angel Javaloyes Victoria (coorientador) - Universidad de Murcia, Espanha

- Prof. Dr. Dirk Töben - UFSCar

- Prof. Dr. José Barbosa Gomes - UFJF

- Prof. Dr. Miguel Domínguez Vázquez - UAM, Espanha 


\section{Agradecimentos}

Dedico esta tese a minha família, amigos e a minha companheira Patricia.

Sou muito grato a Marcos Alexandrino e Miguel Angel pela orientação e amizade. Agradeço pela hospitalidade que fui recebido em Murcia, por Miguel Angel, e em Madrid, por

Miguel Domínguez. E também agradeço aos amigos e funcionários do IME pelos 6 anos de um ótimo convívio. 


\section{Resumo}

ALVES, B. O. Sobre Folheações Finslerianas singulares. 2017. 66 f. Tese (Doutorado) - Instituto de Matemática e Estatística, Universidade de São Paulo, São Paulo, 2017.

Nesta tese foi introduzido o conceito de folheação Finsleriana singular, que generaliza ação Finsleriana, submersão Finsler e folheação Finsleriana. O primeiro resultado desta tese afirma que qualquer folheação Finsleriana singular sobre uma variedade Randers com data $(h, W)$ é folheação Riemanniana singular com respeito a $h$ e $W$ é um campo folheado. Para obter este resultado provou-se um teorema de redução ao slice, que permite relacionar uma folheação Finsleriana singular com uma folheação Finsleriana singular em um espaço de Minkowski. O terceiro resultado garante a equifocalidade para as fibras regulares de uma submersão singular analítica que na parte regular é uma submersão Finsleriana. No transcurso do trabalho verificou-se propriedades relevantes das folheações Finslerianas singulares e a existência de vizinhanças tubulares Finslerianas, uma propriedade básica que não estava escrita na literatura.

Palavras Chave: Folheações Finslerianas singulares; espaços Randers; submersões Finslerianas; equifocalidade. 


\section{Abstract}

ALVES, B.O. On Singular Finsler Foliations. 2017. 66 f. Thesis - Instituto de Matemática e Estatística, Universidade de São Paulo, São Paulo, 2017.

In this thesis we introduce the concept of singular Finsler foliation, which generalizes the concepts of Finsler actions, Finsler submersions and Finsler foliations. In the first theorem we show that if $F$ is a singular Finsler foliation on a Randers manifold $(M, Z)$ with Zermelo data $(h, W)$, then $F$ is a singular Riemannian foliation on the Riemannian manifold $(M, h)$. In order to prove this theorem we present in the second theorem a slice reduction that relates local singular Finsler foliations on Finsler manifolds with singular Finsler foliations on Minkowski spaces. Finally in the third result we prove the equifocality of the fibers of an analytic singular submersion that is a Finsler submersion in the regular part. Along the work we stress relevant properties on singular Finsler foliations and we also remark the existence of tubular neigborhoods in Finsler geometry, a basic result that we did not find properly written in the literature.

Keywords: Singular Finsler foliation, Randers space, Finsler submersion, equifocality. 


\section{Sumário}

1 Geometria Finsleriana $\mathbf{1 5}$

1.1 Métrica Finsler e Tensor Fundamental . . . . . . . . . . . . . . . 15

1.2 Cone Ortogonal . . . . . . . . . . . . . . . . 16

1.3 Função Distância e Geodésica . . . . . . . . . . . . . . . . 17

1.5 Métricas de Zermelo e de Randers . . . . . . . . . . . . . . . . 18

1.10 Conexão de Chern . . . . . . . . . . . . . . . . . . . 23

1.11.1 Exponencial e Campos de Jacobi . . . . . . . . . . . . . . 25

1.13 Vizinhanças Tubulares . . . . . . . . . . . . . . . . 26

1.14 Submersão Finsleriana . . . . . . . . . . . . . . . . . . . 29

1.15 Teorema de Wang caso Finsler . . . . . . . . . . . . . . . . 33

2 Folheações Finslerianas Singulares, Definições e Propriedades 37

2.1 Folheação Finsleriana Regular . . . . . . . . . . . . . . . . . . 38

2.2 Folheação Finsleriana Singular . . . . . . . . . . . . . . . . . . 39

2.5 Propriedades das Folheações Finslerianas Singulares . . . . . . . . . . . . . 43

2.5.1 Estrutura Tubular . . . . . . . . . . . . . . . . 44

2.6.1 Lema de Homotetia . . . . . . . . . . . . . . . . 46

2.7.1 Teorema do Slice e Folheação Infinitesimal . . . . . . . . . . . . . 48

2.7 .2 Estratificação . . . . . . . . . . . . . . . . . 50

3 Folheações Randers Singulares

3.1 Casos Particulares . . . . . . . . . . . . . . . . 53

3.2 Exemplos Não-Homogêneos. . . . . . . . . . . . . . . . 55

3.3 Folheação Finsleriana singular em espaços Randers-Minkowski . . . . . . . . 55

3.4 Prova do Teorema $1 \ldots \ldots \ldots \ldots$. . . . . . . . . . . . . . . . . . . . .

4 Submersão Finsleriana Singular $\quad 57$

4.1 Distribuições de Wilking . . . . . . . . . . . . . . . . . . . 57

4.1.1 Operador Forma e Campos P-Jacobi . . . . . . . . . . . . 57

4.1 .2 Distribuições de Wilking . . . . . . . . . . . . . 59

4.3 Prova do Teorema $3 \ldots \ldots \ldots \ldots$. . . . . . . . . . . . . . . . . .

$\begin{array}{ll}\text { Referências Bibliográficas } & 65\end{array}$ 


\title{
Lista de Símbolos
}

\author{
$g_{v} \quad$ tensor fundamental \\ $K \quad$ curvatura flag \\ $C \quad$ tensor de Cartan \\ $\nabla \quad$ conexão de Chern \\ $\mathcal{F}$ folheação singular \\ $\mathcal{I}^{F} \quad$ indicatriz da métrica Finsler $F$ \\ $\Sigma_{r} \quad$ estrato $r$-dimensional \\ $(h, W)$ data de uma métrica Randers \\ $h_{W} \quad$ métrica Randers com data $(h, W)$ \\ $\nu_{p}(L) \quad$ cone ortogonal à subvariedade $L$ em $p$ \\ $\nu(L) \quad$ cone ortogonal
}




\section{Introdução}

Navegar por um mar calmo com uma brisa é muito bonito: vela estendida, o vento no rosto, timão na mão... A escolha do trajeto define o quanto tardará para chegar ao destino e, ao contrário de uma caminhada no plano, não necessariamente gastaremos menos tempo se seguirmos em linha reta. Qual é o trajeto que minimiza o tempo de viagem? Este problema foi sugerido por Zermelo, por isso é denominado "Problema de Navegação de Zermelo", e solucionado por Shen. A solução é uma geodésica de uma métrica Finsler gerada pela translação da métrica Riemanniana do ambiente pelo vento. Esta métrica é chamada métrica Randers e o par que a define, composto pela métrica Riemanninana ambiente e o vento, é a data. O tema do resultado principal desta tese diz respeito a folheações Finslerianas singulares em uma variedade com uma métrica Randers.

Uma métrica Finsleriana é uma aplicação que a cada ponto e direção em uma variedade associa um produto interno. A dependência da direção (o que não acontece no caso Riemanniano) gera casos em que o comprimento de um vetor difere do comprimento do seu oposto, consequentemente o tempo gasto para percorrer um trajeto depende da direção. Outra diferença é que o espaço normal a uma subvariedade não é um espaço vetorial e possui apenas uma estrutura de cone.

Folheação Finsleriana singular é uma folheação singular sobre uma variedade Finsleriana que cumpre a propriedade de transnormalidade com respeito à métrica Finsleriana do ambiente, ou seja, se uma geodésica é ortogonal a uma folha, então ela é ortogonal a todas as folhas que encontrar. Os primeiros exemplos são: fibras de uma submersão Finsleriana e órbitas de uma ação Finsleriana, chamada de folheação homogênea.

Uma submersão Finsleriana é uma submersão entre variedades Finslerianas tal que infinitesimalmente projeta a bola do espaço total sobre a bola na base. Uma folheação Finsleriana regular é uma folheação localmente descrita por submersões Finslerianas satisfazendo uma condição de cociclo, ou equivalentemente é folheação Finsleriana singular cujas folhas possuem a mesma dimensão (Proposição 2.7.1). Em [18] foi provado que qualquer folheação Finsleriana regular é uma folheação Riemanniana para alguma métrica Riemanniana, usando uma média na base das submersões Finslerianas que descrevem a folheação em questão. Este resultado motivou a seguinte conjectura:

Conjectura 1. Toda folheação Finsleriana singular é uma folheação Riemanniana singular com respeito a alguma métrica Riemanniana.

Em variedades Randers, a ação é isométrica Finsler se, e somente se, for isométrica com respeito à métrica Riemanniana da data e o vento invariante pela ação. Também verifica-se que uma submersão entre espaços Randers é Finsleriana se, e somente se, for uma folheação Riemanniana com respeito às métricas Riemannianas das datas e o vento no espaço total é projetável. Estes fatos sugeriram o seguinte resultado que resolve a conjectura no caso de variedades Randers. 
Teorema 1. Se $\mathcal{F}$ é uma folheação Finsleriana singular em uma variedade Randers com data $(h, W)$ com folhas fechadas, então $\mathcal{F}$ é folheação Riemanniana singular com respeito à métrica Riemanniana $h$ e $W$ é um campo folheado.

Este teorema também fornece uma generalização parcial do problema de navegação de Zermelo. A veracidade da recíproca deste resultado será verificada para folheações Randers regulares, para folheações homogêneas em espaços Randers e espaços Randers com curvaturas flag constante, vide subseção 3.1.

Para provar o Teorema 1, se fez necessário estudar a estrutura local e infinitesimal das folheações Finslerianas singulares. Eis que surgem algumas dificuldades extras tendo em mente o caso Riemanniano. A aplicação exponencial é apenas de classe $C^{1}$ na origem, o espaço normal a uma subvariedade tem apenas uma estrutura de cone, possivelmente não é suave na origem e a função distância não é simétrica. Estas dificuldades nos colocam em alerta na hora de obter no contexto Finsleriano propriedades análogas ao caso Riemanniano. Por exemplo, ao contrário do caso Riemanniano, as "esferas concêntricas" de um espaço de Minkowski (modelo infinitesimal de variedade Finsler) não-reversível não constitui uma folheação Finsleriana singular. Apesar destas dificuldades, serão generalizados: o resultado que garante a existência de vizinhanças tubulares para algumas subvariedades mergulhadas (Teorema 1.13.1), a caracterização das folheações Finslerianas singulares via estruturas tubulares (Proposição 2.6.1), o Lema de Homotetia (Proposição 2.6.2), algumas propriedades de estratificação e o Teorema do Slice (Teorema 2.0.2). Para o estudo de tais resultados no contexto Riemanniano consulte [1], [3], [5], [21].

A existência de vizinhanças tubulares é um resultado secundário nesta tese, porém tais objetos desempenham um papel extremamente fundamental para a teoria aqui desenvolvida. Por conta do problema na diferenciabilidade da exponencial, não é possível usar um argumento análogo ao caso Riemanniano, que usa o Teorema da Função Inversa, vide [19] Lang.

Também fundamental para a teoria das folheações Finslerianas singulares o Teorema de Slice 2.0.2 fornece a estrutura infinitesimal destes objetos, relacionando a geometria transversal das folhas com uma folheação Finsleriana singular em um espaço de Minkowski. Ele desempenha um papel chave na demonstração do Teorema 1.

Teorema 2 (Slice). Seja $(M, F, \mathcal{F})$ uma folheação Finsleriana singular. Então para qualquer $q \in M$ existem uma vizinhança $U$ de uma placa $P_{q}$, um slice $S=S_{q}$ de $\left(U,\left.\mathcal{F}\right|_{U}\right)$ passando por q e uma métrica Finsleriana $Z_{S}$ em $S$ tais que a folheação slice $\mathcal{F}_{q}:=\{L \cap S\}$ é uma folheação Finsleriana singular em $\left(S, Z_{S}\right)$, q é uma folha 0 -dimensional e a equação abaixo é satisfeita:

$$
d_{F}\left(P_{p}, P_{z}\right)=d_{Z_{S}}\left(P_{p} \cap S, P_{z} \cap S\right),
$$

para quaisquer placas $P_{p}$ e $P_{z}$ de $(M, F, \mathcal{F})$ com $p, z \in S$. Além disso, existe um difeomorfismo folheado $f:\left(S_{q}, Z_{S}, \mathcal{F}_{q}\right) \rightarrow\left(\mathbb{R}^{n}, F, \mathcal{F}_{0}\right)$ tal que $f(q)=0$, onde $\left(\mathbb{R}^{n}, F, \mathcal{F}_{0}\right)$ é uma folheação Finsleriana singular em um espaço de Minkowski.

Outra propriedade muito importante é a equifocalidade. Em [26] Terng e Thorbergsson introduziram o conceito de subvariedade equifocal com seções em um espaço simétrico compacto. Tal conceito é uma generalização natural de subvariedades isoparamétricas em espaços Euclidianos. Em [1] Alexandrino e Töben tiraram a condição da existência de seções da definição de equifocalidade e provaram que as folhas regulares de uma folheação Riemanniana singular são equifocais.

Uma folha regular $L$ de uma folheação singular é equifocal quando dado um campo básico $\xi$ ao longo de $L$ a aplicação end-point $\eta_{\xi}(p):=\exp \xi(p)$ tem posto constante. 
Teorema 3. Seja $\pi:(M, F) \rightarrow B$ uma submersão Finsleriana singular, isto é, uma aplicação tal que $\mathcal{F}_{\pi}=\left\{\pi^{-1}(q)\right\}$ restrita ao conjunto regular é uma folheação Finsleriana. Se $\pi$ é analítica, então $\mathcal{F}$ é equifocal.

A principal técnica utilizada na demonstração deste resultado envolve as distribuições de Wilking. Foi preciso generalizar tal conceito para o contexto Finsleriano. Para ler sobre estas distribuições no caso Riemanniano basta consultar [13].

Os resultados obtidos aqui apresentados encontram-se no formato de preprint no arxiv, vide [4] e [8].

No primeiro capítulo apresentaremos preliminarmente tópicos fundamentais em geometria Finsleriana necessários para nossa proposta e também a demonstração da existência de vizinhanças tubulares. No segundo capítulo, introduziremos o conceito de folheação Finsleriana singular e as propriedades fundamentais mencionadas acima. No terceiro capítulo provaremos o Teorema 1. No último capítulo serão introduzidas as distribuições de Wilking e com esta ferramenta será demonstrado o Teorem 3. 


\section{Capítulo 1}

\section{Geometria Finsleriana}

Neste capítulo, com base em [7], [9], [14], [15], [17], [25] serão introduzidos alguns tópicos da geometria Finsleriana necessários à demonstração de nossos resultados. Além de conter as definições de elementos fundamentais da geometria Finsleriana, tais como tensor fundamental, cone ortogonal, geodésica, metrica Randers e funções transnormais (enunciando o Teorema de Wang versão Finsler), está presente neste capítulo a demonstração da existência de vizinhança tubular.

Dada $P$ uma subvariedade em uma variedade Finsleriana, uma vizinhança tubular de $P$ de raio $r$ é um aberto $U$ contendo $P$ tal que a exponencial restrita ao fibrado de bolas abertas de raio $r$ sem a seção nula é um difeomorfismo em $U \backslash P$. Em particular, dado $p \in U$ existe uma única geodésica ortogonal a $P$ contida em $U$ realizando a distância de $P$ a $p$. Será demonstrado que qualquer $P$ pré-compacto de uma subvariedade mergulhada admite uma vizinhança tubular. A demonstração para o caso Riemanniano, vide [19], não pode ser generalizada para o caso Finsleriano. A principal dificuldade está no fato de que a exponencial não necessariamente é um difeomorfismo em um entorno da origem. Este fato foi usado para encontrar um número $i>0$ tal que todas as geodésicas unitárias que partem ortogonalmente a $P$ minimizam a distância até tempo $t=i$.

Outro tema importante aqui tratado é das submersões Finslerianas, conceito fundamental para a teoria das folheações Finslerianas singulares, pois descrevem localmente as folheações Finslerianas regulares.

\subsection{Métrica Finsler e Tensor Fundamental}

Seja $F: V \rightarrow[0, \infty)$ uma função positiva em um espaço vetorial finito dimensional $V$. Dizemos que $F$ é uma norma de Minkowski quando for positiva homogênea (isto é, $F(\lambda v)=\lambda v$ para todos $\lambda>0$ e $v \in V$ ), suave em $V \backslash 0$ e fortemente convexa, ou seja, o tensor fundamental associado a $F$, definido por

$$
g_{v}(u, w):=\left.\frac{1}{2} \frac{\partial^{2}}{\partial s \partial t} F^{2}(v+s u+t w)\right|_{s, t=0}
$$

para quaisquer $v, u, w \in V$ com $v \neq 0$, é positivamente definido. $\mathrm{O}$ par $(V, F)$ é denominado espaço de Minkowski.

Definição 1.1.1. Uma norma (métrica) Finsleriana em uma variedade diferenciável $M$ é uma função contínua $F: T M \rightarrow[0, \infty)$ tal que $\left.F\right|_{T M \backslash 0}: T M \backslash 0 \rightarrow[0, \infty)$ é suave e $F_{p}:=\left.F\right|_{T_{p} M}$ é uma norma de Minkowski para qualquer $p \in M$. 
Dado $v$ não nulo não necessariamente $F(v)$ coincide com $F(-v)$. Quando $F(v)=F(-v)$ para qualquer $v \in V$ a métrica Finsleriana $F$ é dita reversivel.

Verifica-se que, para qualquer $v \in T M \backslash 0$, o tensor fundamental de uma variedade Finsleriana $(M, F)$ possui as seguintes propriedades

(i) $g_{\lambda v}=g_{v}$ para qualquer $\lambda>0$;

(ii) $g_{v}(v, v)=F^{2}(v)$;

(iii) $g_{v}(v, w)=\left.\frac{1}{2} \frac{\partial}{\partial s} F^{2}(v+s w)\right|_{s=0}, w \in T_{\pi(v)} M$.

A indicatriz em $p \in M$ de raio $r>0$ de uma métrica Finsleriana $F: M \rightarrow \mathbb{R}$ é o conjunto

$$
\mathcal{I}_{p}^{F}(r)=\left\{v \in T_{p} M ; F(v)=r\right\} .
$$

A indicatriz, quando não houver risco de confusão, será denotada por $\mathcal{I}_{p}(r)$ e quando $r=1$ por $\mathcal{I}_{p}^{F}$ e neste caso será denominada apenas por indicatriz. O resultado abaixo mostra que $\mathcal{I}^{F}$ determina a métrica $F$. Para a prova deste teorema veja [17].

Teorema 1.1.1. Sejam $V$ um espaço vetorial e $B$ é um subconjunto fechado de $V$ contendo a origem. Suponha que B satisfaz as seguintes condições

1. B é estrelado com respeito a origem;

2. B intersecta todas as direções;

3. $S:=\partial B$ é uma hipersuperfície fechada de $V$ tal que qualquer $v \in S$ é transversal;

4. para qualquer $v \in V \backslash 0$ existe um único $\lambda_{v}>0$ tal que $\frac{v}{\lambda_{v}} \in S$;

5. S fortemente convexa.

Então a aplicação $F: V \rightarrow \mathbb{R}$ que associa a cada $v \in V$ o número $\lambda_{v}$ dado no item (4) é uma métrica de Minkowski cuja indicatriz é $S$. Reciprocamente, se $F: V \rightarrow \mathbb{R}$ é uma métrica de Minkowski, então $B:=F^{-1}[0,1]$ satisfaz os cinco itens acima e neste caso $S=\mathcal{I}^{F}$.

O tensor fundamental de uma variedade Finsleriana $(M, F)$ fornece uma identificação do fibrado tangente com o seu dual. O fibrado dual a $M$ tem por espaço total a variedade $T M^{*}=\cup T_{p} M^{*}$ que é munida da métrica Finsleriana $F_{p}^{*}(\beta)=\sup \left\{\beta(v) \in \mathbb{R} ; v \in \mathcal{I}_{p}^{F}\right\}$, chamada de métrica dual.

A aplicação de Legendre é a aplicação $\mathcal{L}: T M \backslash 0 \rightarrow T M^{*} \backslash 0$ que associa a cada $v \in T_{p} M \backslash 0$ o funcional linear $\mathcal{L}(v)=\mathcal{L}_{v}: T_{p} M \rightarrow \mathbb{R}$ dado por $w \mapsto \mathcal{L}_{v}(w):=g_{v}(v, w)$. Esta aplicação é um difeomorfismo positivo homogêneo de grau 1 que se estende continuamente para 0 tal que $F=F^{*} \circ \mathcal{L}$. Para mais detalhes veja o capítulo $\mathrm{X}$ em [25].

\subsection{Cone Ortogonal}

Dada uma subvariedade $L$ de $M$ um vetor $v \in T_{p} M \backslash 0$ é ortogonal a $L$ quando $p \in L$ e $g_{v}(v, w)=0$ para qualquer $w \in T_{p} L$. O espaço dos vetores ortogonais a $L$ em $p$ é denominado cone ortogonal a $L$ em $p$ e será denotado por $\nu_{p}(L)$. A união disjunta de todos os cones ortogonais a $L$ denotado por $\nu(L):=\cup_{p \in L} \nu_{p}(L)$ será referido como fibrado normal. Para cada intervalo $J$ em $(0, \infty)$ defina

$$
\nu_{p}^{J}(L):=\nu_{p}(L) \cap F^{-1}(J) \text { e } \nu^{J}(L):=\cup_{p \in L} \nu_{p}^{J}(L),
$$


onde $J$ pode ser um intervalo degenerado, isto é, pode conter apenas um número real positivo.

Observe que por definição o zero não está contido no cone ortogonal. Além disso, se $n$ é ortogonal a uma subvariedade $L$, não necessariamente $-n$ é ortogonal a $L$. Logo não necessariamente o cone ortogonal em um ponto $p \in L$ possui uma estrutura de espaço vetorial, mesmo incluindo o zero, e consequentemente o fibrado normal quase nunca é um fibrado vetorial.

Exemplo 1.2.1. Seja $(M, F)$ uma variedade Finsleriana. Qualquer vetor $v$ em $T_{p} M$ está no cone ortogonal à indicatriz $\mathcal{I}_{p}(r) \subset\left(T_{p} M, F_{p}\right), r=F(v)$. De fato, dada uma curva suave $\gamma:(-\epsilon, \epsilon) \rightarrow \mathcal{I}_{p}$ com $\gamma(0)=v$ temos que $F(\gamma)=r$, logo

$$
0=\left.\frac{d}{d s}\right|_{s=0} F^{2}(\gamma(s))=d F_{v}^{2}\left(\gamma^{\prime}(0)\right)=\left.\frac{d}{d t}\right|_{t=0} F^{2}\left(v+t \gamma^{\prime}(0)\right)=g_{v}\left(v, \gamma^{\prime}(0)\right),
$$

e isto mostra, pela generalidade da curva $\gamma \subset \mathcal{I}_{p}(r)$, que $v$ está no cone ortogonal a $\mathcal{I}_{p}(r)$ em $v$.

Dada $L$ uma subvariedade $k$-dimensional de $\left(M^{n}, F\right)$, seja $\mathcal{N}_{p}=A n n\left(T_{p} L\right)$ o anulador de $T_{p} L$ em $T_{p} M$ (ou seja, $\operatorname{Ann}\left(T_{p} M\right):=\left\{\beta \in T_{p} M^{*} ;\left.\beta\right|_{T_{p} L}=0\right\}$ ) que é um subespaço vetorial $(n-k)$-dimensional de $T M^{*}$. A seção $\mathcal{N}$ de $\pi^{-1}(L)^{*}$ que associa a cada $p \in L$ o anulador $\mathcal{N}_{p}=\operatorname{Ann}\left(T_{p} L\right)$ é um subfibrado vetorial de $\pi^{-1}(L)^{*} \rightarrow L$.

Lema 1.2.1. Se L é uma k-subvariedade de uma variedade Finsleriana $\left(M^{n}, F\right)$, então $\nu_{p}(L)$ e $\nu_{p}^{r}(L), \nu(L)$ e $\nu^{r}(L)$ são subvariedades de dimensões $n-k, n-k-1$, n e $n-1$, respectivamente, para qualquer $r>0$. Além disso, $\left.\rho\right|_{\nu^{r}(L)}: \nu^{r}(L) \rightarrow L$ e $\left.\rho\right|_{\nu(L)}: \nu(L) \rightarrow L$ são submersões.

Demonstração. Observe que $\nu_{p}(L)=\mathcal{L}^{-1}\left(\mathcal{N}_{p}\right)$ e $\nu_{p}^{r}(L)=\mathcal{L}^{-1}\left(\mathcal{N}_{p} \cap\left(F^{*}\right)^{-1}(r)\right)$ para cada $p \in L$; e $\nu(L)=\mathcal{L}^{-1}(\mathcal{N})$ e $\nu^{r}(L)=\mathcal{L}^{-1}\left(\mathcal{N} \cap\left(F^{*}\right)^{-1}(r)\right), r>0$. Logo como $\mathcal{N}_{p}$ é uma $(n-k)$ variedade e $\mathcal{L}$ é um difeomorfismo segue que $\nu_{p}(L)$ e $\nu_{p}^{r}(L), \nu(L)$ e $\nu^{r}(L)$ são subvariedades de dimensões $n-k, n-k-1, n$ e $n-1$, respectivamente.

Resta verificar a segunda proposição do resultado. O conjunto $\nu^{r}(L)$ pode ser escrito localmente como o produto $\nu_{p}^{r}(L) \times U$ para algum $U$ aberto de $L$. Neste caso, $\rho(v, q)=q$ para qualquer $(v, q) \in \nu_{p}^{r}(L) \times U$ e portanto $\left.\rho\right|_{\nu^{r}(L)}$ é uma submersão. Analogamente prova-se que $\left.\rho\right|_{\nu(L)}$ também é uma submersão.

\subsection{Função Distância e Geodésica}

Dada uma variedade Finsleriana $(M, F)$ define-se uma estrutura de comprimento considerando o funcional comprimento que associa a cada curva suave por partes $\gamma:[a, b] \rightarrow M$ o número real

$$
L(\gamma)=\int_{a}^{b} F\left(\gamma^{\prime}(s)\right) d s .
$$

Já a distância entre $p$ e $q$ dois pontos quaisquer em $(M, F)$ é definida por

$$
d(p, q)=d_{F}(p, q)=\inf \{L(\gamma) ; \gamma \in C(p, q)\}
$$

onde o ínfimo é tomado sobre $C(p, q)$ espaço das curvas suave por partes ligando $p$ a $q$. Observe que esta distância não necessariamente é simétrica, ou seja, em geral, $d(p, q) \neq$ $d(q, p)$. Consequentemente para cada $p \in M$ e $r>0$ temos dois tipos de bola; a bola futura $B^{+}(p, r)=\{q \in M ; d(p, q)<r\}$ e a bola passada $B^{-}(p, r)=\{q \in M ; d(q, p)<r\}$. 
Uma curva é uma geodésica quando a norma da velocidade é constante e é ponto crítico do funcional energia

$$
E_{F}(\gamma)=\int_{a}^{b} F^{2}(\gamma(s)) d s
$$

definido no espaço das curvas suaves por partes $\gamma:[a, b] \rightarrow M$ tais que $\gamma(a)=p$ e $\gamma(b)=q$. Verifica-se que uma curva suave por partes é ponto crítico do funcional comprimento se, e somente se, for uma reparametrização de uma geodésica.

Exemplo 1.3.1. A geodésica em um espaço de Minkowski $V$ que parte de $p \in V$ com velocidade $v$ é a reta $\gamma(t)=p+t v$.

Para cada $v \in T_{p} M$ existe uma única geodésica $\gamma_{v}: I_{v} \rightarrow M$ tal que $\gamma_{v}^{\prime}(0)=v$, onde $I_{v}$ é o intervalo máximo de definição de $\gamma_{v}$. Além disso, existe $\epsilon>0$ tal que $d\left(p, \gamma_{v}(t)\right)=L\left(\left.\gamma_{v}\right|_{(0, t)}\right)$ para qualquer $t \in(0, \epsilon)$. E também para cada ponto $p \in M$ existe $U \subset M$ uma vizinhança de $p$ tal que para qualquer $q \in U$ existe um único segmento de geodésica minimizante de $p$ a $q$.

Observação 1.4. É importante observar que, ao contrário do caso Riemanniano, se $\gamma$ é uma geodésica, então não necessariamente $s \mapsto \gamma(-s)$ é uma geodésica.

Dada uma subvariedade $P$ de $(M, F)$ definem-se as seguintes funções distâncias

$$
\rho_{+}(q):=d(P, q):=\inf _{p \in P} d(p, q) \text { e } \rho_{-}(p):=d(p, P):=\inf _{q \in P} d(p, q) .
$$

Em geral $\rho_{+}$e $\rho_{-}$são distintos, ao contrário do caso Riemanniano.

Lema 1.4.1. Sejam $(M, F)$ uma variedade Finsleriana e $P$ uma subvariedade fechada em $M$. Se $\gamma:[0,1] \rightarrow M$ é uma geodésica tal que $L(\gamma)=d(P, \gamma(1))$, então $\gamma^{\prime}(0) \in \nu(P)$.

\subsection{Métricas de Zermelo e de Randers}

Nesta seção será apresentado um exemplo de métricas Finsler que é o ambiente do principal resultado desta tese. Tal exemplo é a métrica Randers, que aparece naturalmente em muitas aplicações, vide [12], [17], [20]. Classicamente, uma métrica Randers em uma variedade Riemanniana $M$ é uma aplicação $R: T M \rightarrow \mathbb{R}$ definida por $R(v)=\alpha(v)+\beta(v)$, onde $\alpha$ e $\beta$ são respectivamente uma métrica Riemanniana e uma 1-forma em $M$ tais que $\|\beta\|_{\alpha}<1$, com $\|.\|_{\alpha}$ norma no espaço dual induzida por $\alpha$. A família das métricas Randers coincide com outra família de métricas, chamadas de métricas de Zermelo.

O problema de navegação de Zermelo consiste em encontrar a trajetória que minimiza o tempo de viagem de uma partícula em uma variedade Riemanniana $(M, h)$ sob a ação de um campo $W$ cuja a norma Riemanniana não excede uma unidade. Existe uma única solução para o problema de navegação de Zermelo com data $(h, W)$ que é a métrica Finsleriana $Z: T M \rightarrow \mathbb{R}$, chamada de métrica de Zermelo, dada implicitamente pela equação $\mid \frac{v}{Z(v)}-$ $W \mid=1, v \in T M \backslash 0$, onde $||=.\sqrt{h(\cdot, \cdot)}$.

Observação 1.6. Mais geralmente, se $F: T M \rightarrow \mathbb{R}$ é uma métrica Finsleriana e $W$ um campo suave em $M$ com $F(W)<1$, define-se a translação de $F$ por $W$ como a métrica Finsleriana $F_{W}: T M \rightarrow \mathbb{R}$ definida implicitamente pela equação

$$
F\left(\frac{v}{F_{W}(v)}-W\right)=1, v \in T M \backslash 0 .
$$


Observe que as indicatrizes destas métricas são relacionadas por

$$
\mathcal{I}^{F_{W}}=\mathcal{I}^{F}+W
$$

Em particular, a métrica de Zermelo $Z: T M \rightarrow \mathbb{R}$ com data $(h, W)(h(W, W)<1)$ é a translação da norma Riemanniana $\sqrt{h}$ pelo campo $W$. Em símbolos, $Z=(\sqrt{h})_{W}$.

Lema 1.6.1. Uma aplicação $Z: T M \rightarrow \mathbb{R}$ é uma métrica de Zermelo com data $(h, W)$ se, e somente se, for a métrica Randers

$$
\alpha+\beta=\sqrt{a}+\beta
$$

onde a e $\beta$ são respectivamente métrica Riemanniana e 1-forma dadas por

$$
a(u, v)=\frac{\lambda h(u, v)+h(u, W) h(v, W)}{\lambda^{2}} \quad \text { e } \beta(v)=-\frac{h(v, W)}{\lambda}
$$

com $\lambda=1-h(W, W)$. Além disso,

$$
h(v, u)=\lambda(a(v, u)-a(B, v) a(B, u))
$$

onde $B$ é o campo definido por $W=-\frac{B}{\lambda} \operatorname{com} \lambda=1-a(B, B)$.

Demonstração. Suponha que $Z$ é uma métrica de Zermelo. Seja $u \in T M$ tal que $h(u, u)=1$. Escreva $u=\frac{v}{Z(v)}-W$. Por definição, $h\left(\frac{v}{Z(v)}-W, \frac{v}{Z(v)}-W\right)=1$, logo, denotando $\lambda=$ $1-h(W, W)$ e usando apenas a bilinearidade de $h$, obtém-se que

$$
Z(v)^{2}+\frac{2 h(v, W)}{\lambda} Z(v)-\frac{h(v, v)}{\lambda}=0 .
$$

E resolvendo esta equação do segundo grau em termos de $Z(v)$, conclui-se que

$$
Z(v)=\sqrt{\frac{\lambda h(v, v)+[h(v, W)]^{2}}{\lambda^{2}}}-\frac{h(v, W)}{\lambda} .
$$

O resultado segue pela 1-homogeneidade de $Z$.

Reciprocamente, dada uma métrica Randers $Z=\alpha+\beta$, defina a métrica Riemanniana $h$ pela equação 1.6 e o campo $W=-\frac{B}{\lambda}$, onde $\alpha=\sqrt{a}, B$ é o campo $a$-dual a $\beta$ (ou seja, $\beta=a(B)$,$) e \lambda=1-a(B, B) 1$. Para $v \in T M$ tal que $Z(v)=1$,

$$
\begin{aligned}
h(v-W, v-W) & =h(v, v)-2 h(v, W)+h(W, W) \\
& =a(v, v)-a(B, v)^{2}-2(a(v, W)-a(B, v) a(B, W))+a(W, W)-a(W, B)^{2} \\
& =\alpha(v)^{2}-\beta(v)^{2}-2\left(\frac{1}{\lambda}(-\beta(v)+\beta(v) a(B, B))\right)+a(W, W)-a(W, B)^{2}=1
\end{aligned}
$$

pois $a(W, W)-a(W, B)^{2}=0$ e $\alpha(v)^{2}-\beta(v)^{2}=\alpha(v)-\beta(v)$, usando que $Z(v)=1$.

Observação 1.7. Na seção 1.14 será definida a translação de uma submersão Finsleriana e na Proposiçõa 3.1.1 será provado que uma submersão entre espaços Randers é Finsleriana se, e somente se, é uma submersão com respeito às métricas das datas e o vento no ambiente se projeta no vento na base. Neste sentido, isso é uma generalização do Lema anterior, assim como o resultado principal Teorema 1 também o é. 
O lema a seguir relaciona a aplicação de Legendre de uma métrica Randers $Z=\left(h_{W}\right)$ com a aplicação de Legendre da métrica Riemanniana $h$. Isso permite relacionar o cone normal com o subespaço $h$-ortogonal e $Z$-gradiente de uma função com o $h$-gradiente.

Lema 1.7.1. Seja $Z(\cdot)=\alpha(\cdot)+\beta(\cdot)=\sqrt{a(\cdot, \cdot)}+a(\cdot, B)$ uma métrica Randers com data $(h, W)$ sobre uma variedade $M$. Então dado $v \in T_{p} M \backslash 0$ obtemos as seguintes expressões para a aplicação de Legendre:

$$
\begin{aligned}
\mathcal{L}_{v}(u):=g_{v}(v, u) & =Z(v)\left(\frac{a(u, v)}{\alpha(v)}+\beta(u)\right)=\frac{Z(v)}{\alpha(v)} a(v+\alpha(v) B, u) \\
& =\frac{Z(v)}{\lambda \alpha(v)} h(v-Z(v) W, u),
\end{aligned}
$$

para qualquer $u \in T_{p} M$.

Demonstração. Pela definição de tensor fundamental e pela regra da cadeia segue que

$$
\begin{aligned}
g_{v}(v, u) & =\left.\frac{1}{2} \frac{\partial}{\partial t} Z^{2}(v+t u)\right|_{t=0}=\left.Z(v) \frac{\partial}{\partial t} Z(v+t u)\right|_{t=0} \\
& =\left.Z(v) \frac{\partial}{\partial t}[\alpha(v+t u)+\beta(v+t u)]\right|_{t=0}=Z(v)\left[\left.\frac{\partial}{\partial t} \alpha(v+t u)\right|_{t=0}+\beta(u)\right] \\
& =Z(v)\left[\left.\frac{1}{2 \alpha(v)} \frac{\partial}{\partial t} a(v+t u, v+t u)\right|_{t=0}+\beta(u)\right]=Z(v)\left(\frac{a(u, v)}{\alpha(v)}+\beta(u)\right) .
\end{aligned}
$$

Por outro lado, usando estas mesmas contas e a relação entre $a, \beta$ e $h$ presentes no Lema 1.6.1, temos que

$$
\begin{aligned}
g_{v}(v, u) & =\left.Z(v)\left[\frac{1}{2 \alpha(v)} \frac{\partial}{\partial t} a(v+t u, v+t u)+\beta(u)\right]\right|_{t=0} \\
& =Z(v)\left[\left.\frac{1}{2 \alpha(v)} \frac{\partial}{\partial t} \frac{1}{\lambda^{2}}\left(\lambda h(v+t u, v+t u)+h(v+t u, W)^{2}\right)\right|_{t=0}-\frac{1}{\lambda} h(u, W)\right] \\
& =\frac{Z(v)}{\alpha(v)}\left[\frac{1}{2 \lambda^{2}}(2 \lambda h(v, u)+2 h(v, W) h(u, W))-\frac{\alpha(v)}{\lambda} h(u, W)\right] \\
& =\frac{Z(v)}{\lambda \alpha(v)}\left[\left(h(v, u)+\frac{1}{\lambda} h(v, W) h(u, W)\right)-\alpha(v) h(u, W)\right] \\
& =\frac{Z(v)}{\lambda \alpha(v)} h\left(v+\left(\frac{1}{\lambda} h(v, W)-\alpha(v)\right) W, u\right)=\frac{Z(v)}{\lambda \alpha(v)} h(v-Z(v) W, u) .
\end{aligned}
$$

Em particular, as fórmulas do lema acima permitem relacionar o cone normal a uma subvariedade com os espaços normais induzidos pelas métricas Riemannianas $a$ e $h$.

Observação 1.8. Calculando diretamente da definição, ou usando a fórmula 1.8, verifica-se que o tensor fundamental de uma métrica Randers $Z=\alpha+\beta=\sqrt{a}+\beta$ é dado pela seguinte expressão

$$
g_{v}(w, u)=\frac{Z(v)}{\alpha(v)}\left[a(w, u)-\frac{a(v, w) a(v, u)}{\alpha^{2}(v)}\right]+\left(\frac{a(v, w)}{\alpha(v)}+\beta(w)\right)\left(\frac{a(v, u)}{\alpha(v)}+\beta(u)\right),
$$


para quaisquer $v, u, w \in T_{p} M$ com $v$ não nulo. De fato,

$$
\begin{aligned}
g_{v}(u, w) & :=\left.\frac{1}{2} \frac{\partial^{2}}{\partial s \partial t} F^{2}(v+s u+t w)\right|_{s, t=0}=\left.\frac{\partial}{\partial s} g_{v+s u}(v+s u, w)\right|_{s=0} \\
& =\left.\frac{\partial}{\partial s}\left[Z(v+s u)\left(\frac{a(v+s u, w)}{\alpha(v+s u)}+\beta(w)\right)\right]\right|_{s=0} \\
& \left.=\left.\frac{\partial}{\partial s} Z(v+s u)\right|_{s=0}\left(\frac{a(v, w)}{\alpha(v)}+\beta(w)\right)+Z(v) \frac{\partial}{\partial s}\left(\frac{a(v+s u, w)}{\alpha(v+s u)}+\beta(w)\right)\right]\left.\right|_{s=0} \\
& =\frac{Z(v)}{\alpha(v)}\left[a(w, u)-\frac{a(v, w) a(v, u)}{\alpha^{2}(v)}\right]+\left(\frac{a(v, w)}{\alpha(v)}+\beta(w)\right)\left(\frac{a(v, u)}{\alpha(v)}+\beta(u)\right),
\end{aligned}
$$

pois $\left.\frac{\partial}{\partial s} Z(v+s u)\right|_{s=0}=\frac{a(v, u)}{\alpha(v)}+\beta(u)$.

Exemplo 1.8.1. Seja $\left(M, Z=h_{W}\right)$ variedade Randers com data $(h, W)$. Suponha que $Z=$ $\sqrt{a}+\beta$. Dado $v \in T_{p} M$, segue que $T_{v} \mathcal{I}_{p}(r)=\left\{w \in T_{p} M ; g_{v}(v, w)=0\right\}+v$, onde $r=Z(v)$. Portanto se $v=W(p), T_{v} \mathcal{I}_{p}(r)=\left\{w \in T_{p} M ; a(v, w)=0\right\}+v=\left\{w \in T_{p} M ; h(v, w)=0\right\}+v$, pelo Lema 1.7.1.

Observação 1.9. Seja L uma subvariedade de uma variedade Randers $(M, Z)$ com data $(h, W)$. O Lema 1.7.1 implica que $W(p)$ é $h$-ortogonal a $L$ se, e somente se, $\nu_{p}(L)$ coincide com o o subespaço vetorial $L^{\perp}:=\left\{v ; h(v, w)=0 \forall w \in T_{p} L\right\}$. Em particular, $\left(\nu_{p}(L),\left.Z\right|_{\nu_{p}(L)}\right)$ é um espaço Randers-Minkowski com data $\left(\left.h\right|_{\nu_{p}(L)}, W(q)\right)$.

\section{Vento Infinitesimalmente Homotético}

Nesta subseção será considerado um caso particular apresentado em [24], que engloba as variedades Randers com curvatura flag constante.

Seja $(M, R)$ uma variedade Randers com data $(h, W)$. Um campo suave $W$ em $T M$ é infinitesimalmente homotético quando

$$
\mathcal{L}_{W} h=\sigma h
$$

onde $\sigma$ é uma constante chamada de constante homotética e $\mathcal{L}$ é a derivada de Lie.

Seja $\varphi$ o fluxo do campo $W$, isto é, para todo $p \in M$ existem $U=U_{p}$ vizinhança aberta de $p$ e $\epsilon=\epsilon(p)>0$ tais que está definida a aplicação suave $\varphi:(-\epsilon, \epsilon) \times U \rightarrow M$ com as seguintes propriedades: $\varphi(0, q)=q, \varphi(t+s, q)=\varphi(t, \varphi(s, q))$ e $\left.\frac{d}{d t}\right|_{t=t_{0}} \varphi(t, q)=W\left(\varphi\left(t_{0}, q\right)\right)$, para quaisquer $q \in U$ e $t, s \in(-\epsilon, \epsilon)$ tais que $t+s \in(-\epsilon, \epsilon)$. Em particular,

$$
d \varphi_{t} W(q)=W\left(\varphi_{t}(q)\right)
$$

onde $\varphi_{t}=\varphi(t):, U \rightarrow M$.

Lema 1.9.1. Se o campo $W$ é infinitesimalmente homotético para a métrica Riemanniana $h$ com constante homotética $\sigma$, então $\varphi_{t}^{*} h=e^{\sigma t} h$.

Demonstração. Basta notar que $\left.\frac{d}{d t}\right|_{t=0} e^{\sigma t} h=\sigma h=\mathcal{L}_{W} h:=\left.\frac{d}{d t}\right|_{t=0} \varphi_{t}^{*} h$ e que $\varphi_{0}=I d$.

Teorema 1.9.1. Uma variedade Randers $(M, R)$ com data $(h, W)$ tem curvatura flag constante $K$ se, e somente se, existe $\sigma$ constante tal que

1. $(M, h)$ tem curvatura seccional constante $K+\frac{1}{16} \sigma^{2} ; e$

2. a data $(h, W)$ é infinitesimalmente homotética com constante homotética $\sigma$. 
Exemplo 1.9.1. Os campos infinitesimalmente homotéticos de $\mathbb{R}^{2}$ são da forma

$$
W(u, v)=\left(\frac{1}{2} \sigma u+k v+c_{1}, \frac{1}{2} \sigma v-k u+c_{2}\right)
$$

onde $k, c_{1}, c_{2}$ são constantes. Logo a variedade Randers $\left(\mathbb{R}^{2}, R\right)$ com data $(\langle\rangle, W$,$) possui$ curvatura flag constante igual a $K=-\frac{1}{16} \sigma^{2} \leq 0$. Em particular, espaços Randers-Minkowski 2-dimensionais são infinitesimalmente homotéticos com $\sigma=k=0$.

O teorema a seguir relaciona as geodésicas de uma variedade Randers com vento infinitesimalmente homotético com as geodésicas do ambiente Riemanniano.

Teorema 1.9.2. As geodésicas unitárias $\gamma:(-\epsilon, \epsilon) \rightarrow M$ de $(M, R)$ uma variedade Randers com data $(h, W)$ infinitesimalmente homotética e constante de homotetia $\sigma$ são da forma

$$
\gamma(t)=\varphi(t, \rho(t))
$$

onde $\rho:(-\epsilon, \epsilon) \rightarrow M$ é uma geodésica de $h$ tal que $h\left(\rho^{\prime}(t), \rho^{\prime}(t)\right)=e^{-\sigma t}$ e $\varphi$ é o fluxo de $W$.

\section{Métrica de Fermat}

Nesta subseção será apresentada uma aplicação das métrica Randers a Relatividade, conforme [10], [11], [17]. Uma variedade Lorentziana $(M, g)$ é dita estacionária canônica normalizada se $M=M_{0} \times \mathbb{R}$ e

$$
g\left(\left(v_{1}, t_{1}\right),\left(v_{2}, t_{2}\right)\right)=g_{0}\left(v_{1}, v_{2}\right)+\omega\left(v_{1}\right) t_{2}+\omega\left(v_{2}\right) t_{1}-t_{1} t_{2},
$$

para $\left(v_{i}, t_{i}\right) \in T_{x} M_{0} \times \mathbb{R}$, onde $\left(M_{0}, g_{0}\right)$ é uma variedade Riemanniana e $\omega$ uma 1-forma de $M_{0}$. Observe que neste tipo de espaço a projeção canônica $t: M \times \mathbb{R} \rightarrow \mathbb{R}$ define uma função tempo global, desta forma a primeira coordenada define uma posição espacial.

Fixe duas posições espaciais $x_{0}$ e $x_{1}$ em $M_{0}$. O Princípio de Fermat diz que as geodésicas luminosas são pontos críticos do funcional global tempo definido sobre as trajetórias tipo luz que ligam $\left(x_{0}, t_{0}\right)$ a $\left(x_{1}, t_{1}\right)$. Se $\gamma=(x, t):[0,1] \rightarrow M$ é uma geodésica luminosa de $\left(x_{0}, t_{0}\right)$ a $\left(x_{1}, t_{1}\right)$, será calculado $t_{1}$, que é o valor do tempo global em $\gamma(1)$. Como $\gamma$ é tipo luz

$$
g_{0}\left(x^{\prime}, x^{\prime}\right)+2 \omega\left(x^{\prime}\right) t^{\prime}-t^{\prime 2}=0 .
$$

Logo, assumindo $\gamma$ orientada para o futuro (isto é, $t^{\prime}>0$ ),

$$
t^{\prime}=\sqrt{g_{0}\left(x^{\prime}, x^{\prime}\right)+\omega\left(x^{\prime}\right)^{2}}+\omega\left(x^{\prime}\right) .
$$

Integrando esta equação, obtém-se

$$
t(s)=t_{0}+\int_{0}^{s}\left(\sqrt{g_{0}\left(x^{\prime}, x^{\prime}\right)+\omega\left(x^{\prime}\right)^{2}}+\omega\left(x^{\prime}\right)\right) d v
$$

e consequentemente geodésicas luminosas são pontos críticos do seguinte funcional

$$
T(\gamma):=t_{0}+\int_{0}^{s}\left(\sqrt{g_{0}\left(x^{\prime}, x^{\prime}\right)+\omega\left(x^{\prime}\right)^{2}}+\omega\left(x^{\prime}\right)\right) d v .
$$

A métrica de Fermat associada a $(M, g)$ é a métrica Randers em $M_{0}$ definida por

$$
F(v)=\sqrt{g_{0}(v, v)+\omega(v)^{2}}+\omega(v) .
$$

A seguir as geodésicas luminosas de $(M, g)$ serão relacionadas com as geodésicas em $\left(M_{0}, F\right)$. 
Teorema 1.9.3 (Princípio de Fermat). Seja $(M, g)$ um espaçotempo estacionário canônico. Uma curva $\gamma=(x, t):[a, b] \rightarrow(M, g)$ é uma geodésica luminosa se, e somente se, $x$ : $[a, b] \rightarrow M_{0}$ é uma geodésica para a métrica de Fermat $F$ e

$$
t(\gamma(s))=T(\gamma \mid[a, s])=t_{0}+\int_{a}^{s} F\left(x^{\prime}\right) d v
$$

para qualquer $s \in[a, b]$.

\subsection{Conexão de Chern}

Da necessidade de calcular a derivada do tensor fundamental surge um tensor trilinear dado por

$$
C_{v}\left(w_{1}, w_{2}, w_{3}\right)=\left.\frac{1}{2} \frac{\partial}{\partial s} g_{v+s w_{1}}\left(w_{2}, w_{3}\right)\right|_{s=0}=\left.\frac{1}{4} \frac{\partial^{3}}{\partial s_{3} \partial s_{2} \partial s_{1}} F^{2}\left(v+\sum_{i=1}^{3} s_{i} w_{i}\right)\right|_{s_{1}, s_{2}, s_{3}=0},
$$

para quaisquer $v, w_{1}, w_{2}, w_{3} \in T_{\pi(v)} M \operatorname{com} v$ não nulo. A família $C=\left\{C_{v} ; v \in T M \backslash 0\right\}$ é denominada tensor de Cartan.

Lema 1.10.1. Para qualquer $v \in T_{p} M \backslash 0$, o tensor de Cartan possui as seguintes propriedades

(i) $C_{v}$ é simétrico;

(ii) $C_{v}$ é positivo homogêneo de grau -1 , isto é, $C_{\lambda v}=\frac{1}{\lambda} C_{v}, \lambda>0$;

(iii) $C_{v}(v, u, w)=C_{v}(u, v, w)=C_{v}(u, w, v)=0, u, w \in T_{p} M$.

De certa forma o tensor de Cartan mede o quanto a métrica Finsler não é Riemanniana, pois $C$ é identicamente nulo se, e somente se, o tensor fundamental não depende da direção e neste caso $F$ é uma norma Riemanniana.

Seja $(M, F)$ uma variedade Finsleriana. Dado $V$ um campo suave em um aberto $U \subset M$ sem singularidades, considere uma conexão linear $\nabla^{V}$ no fibrado tangente $T U$. Dizemos que $\nabla^{V}$ é livre de torção quando $[X, Y]=\nabla_{X}^{V} Y-\nabla_{Y}^{V} X, X, Y \in \Gamma(T U)$. E se $\nabla^{V}$ cumpre com a equação

$$
X g_{V}(Y, Z)=g_{V}\left(\nabla_{X}^{V} Y, Z\right)+g_{V}\left(Y, \nabla_{X}^{V} Z\right)+2 C_{V}\left(\nabla_{X}^{V} V, Y, Z\right)
$$

para quaisquer $X, Y, Z \in \Gamma(T U)$, dizemos que $\nabla^{V}$ é quase compativel com a métrica.

Lema 1.10.2. Seja L subvariedade Finsleriana de $(M, F)$ uma variedade Finsler. Então para qualquer $v \in \nu_{p}(L)$ o espaço tangente a $\nu_{p}(L)$ em $v$ coincide com o subespaço $g_{v^{-}}$ ortogonal a $L$ em p. Em simbolos,

$$
T_{v} \nu_{p}(L)=\left\{u \in T_{p} M ; g_{v}(u, w)=0 \forall w \in T_{p} L\right\} .
$$

Demonstração. Para verificar este resultado basta notar que os subespaços em questão possuem a mesma dimensão e que se $\alpha$ é uma curva suave em $\nu_{p}(L)$, então $g_{\alpha}\left(\alpha^{\prime}, w\right)=0$, para qualquer $w \in T_{p} L$ fixado: pela quase-compatibilidade da métrica $g$, e por uma propriedade do Tensor de Cartan

$$
0=\left.\frac{d}{d t}\right|_{t=0} g_{\alpha}(\alpha, w)=g_{\alpha}\left(\alpha^{\prime}, w\right)+g_{\alpha}\left(\alpha, w^{\prime}\right)+2 C_{\alpha}\left(\alpha^{\prime \prime}, \alpha, w\right)=g_{\alpha}\left(\alpha^{\prime}, w\right),
$$

onde $w$ foi considerado como um campo paralelo ao longo de $\alpha$ em $T_{p} M$, isto é, $w^{\prime}=\nabla_{\alpha}^{\alpha} w=$ 0 . 
Proposição 1.10.1. Dado um campo de vetores $V$ sem singularidades definido em um aberto $U \subset M$ existe uma única conexão afim livre de torção e quase g-compativel $\nabla^{V}$. Tal conexão é determinada pela seguinte fórmula tipo Koszul

$$
\begin{aligned}
2 g_{V}\left(\nabla_{X}^{V} Y, Z\right) & =X g_{V}(Y, Z)-Z g_{V}(X, Y)+Y g_{V}(Z, X) \\
& +g_{V}([X, Y], Z)+g_{V}([Z, X], Y)-g_{V}([Y, Z], X) \\
& +2\left(-C_{V}\left(\nabla_{X}^{V} V, Y, Z\right)-C_{V}\left(\nabla_{Y}^{V} V, Z, X\right)+C_{V}\left(\nabla_{Z}^{V} V, X, Y\right)\right) .
\end{aligned}
$$

A família de conexões dada pela proposição acima é chamada de conexão de Chern. Verifica-se que $\nabla^{\lambda V}=\nabla^{V}$ para qualquer $\lambda>0$ e que $\nabla_{p}^{V}$ depende apenas de $v=V(p)$ para qualquer $p \in U$.

Um campo suave $V$ definido em um aberto de $M$ é geodésico quando $\nabla_{V}^{V} V=0$, isto equivale a dizer que suas curvas integrais são geodésicas.

Lema 1.10.3. Seja $(M, F)$ uma variedade Finsleriana. Se $V$ é um campo geodésico definido em um aberto $U$ de $M$, então

$$
\tilde{\nabla}_{V}=\nabla_{V}^{V}
$$

onde $\nabla^{V}$ é a conexão de Chern e $\tilde{\nabla}$ a conexão de Levi-Civita da métrica Riemanniana $g_{V}$.

Demonstração. Pelas formulas de Koszul Finsleriana e Riemanniana, pela propriedade (iii) do Lema 1.10 .1 e pela hipótese de $V$ ser campo geodésico, temos que

$$
\begin{aligned}
2 g_{V}\left(\nabla_{V}^{V} Y, Z\right) & =V g_{V}(Y, Z)-Z g_{V}(V, Y)+Y g_{V}(Z, V) \\
& +g_{V}([V, Y], Z)+g_{V}([Z, V], Y)-g_{V}([Y, Z], V)=2 g_{V}\left(\tilde{\nabla}_{V} Y, Z\right),
\end{aligned}
$$

para quaisquer campos suaves $Y$ e $Z$ definidos em $U$.

Dada uma curva suave $\gamma:[a, b] \rightarrow U$ para uma extensão $V$ de $\gamma$ existe uma única aplicação $D_{\gamma}^{V}: \Gamma\left(\gamma^{*}\right) \rightarrow \Gamma\left(\gamma^{*}\right), X \mapsto D_{\gamma}^{V} X$ tal que

1. $D_{\gamma}^{V}(X+Y)=D_{\gamma}^{V}(X)+D_{\gamma}^{V}(Y)$, para quaisquer $X, Y \in \Gamma\left(\gamma^{*}\right)$;

2. $D_{\gamma}^{V}(f X)=f^{\prime}\left(t_{0}\right) X\left(t_{0}\right)+f\left(t_{0}\right) D_{\gamma}^{V} X$ para quaisquer $f \in \mathcal{F}([a, b]), X \in \Gamma\left(\gamma^{*}\right)$;

3. $D_{\gamma}^{V} X(t)=\nabla_{\gamma^{\prime}(t)}^{V} X$ para qualquer $t \in[a, b]$ e $X \in \Gamma(T U)$.

A derivada covariante de $\gamma$ é a aplicação $D_{\gamma}^{\gamma^{\prime}}:=D_{\gamma}^{V}: \Gamma^{*}(\gamma) \rightarrow \Gamma^{*}(\gamma)$, que está bem definida, pois não depende da escolha da extensão $V$, pela Proposição 2.15 em [15]. Dado $X$ um campo ao longo de $\gamma$, quando não houver o risco de confusão, será usada a notação: $X^{\prime}=D_{\gamma}^{\gamma^{\prime}} X$.

Dado um campo $V$ sem singularidades, considere $\left\{E_{i}\right\}$ um referencial local $g_{V^{-}}$ortonormal. A derivada covariante de $X$ ao longo de $\gamma$ com respeito a $V$ é escrita nestas coordenadas da seguinte forma

$$
D_{\gamma}^{V} X(t)=\frac{d}{d t} X^{i}(t) E_{i}+X^{i}(t) \gamma^{i^{\prime}}(t) \Gamma_{i j}^{k}(V(t)) E_{k},
$$

onde $\Gamma_{i j}^{k}(V):=g_{V}\left(\nabla_{E_{i}}^{V} E_{j}, E_{k}\right)$ são os símbolos de Christoffel que dependem suavemente de $V(t)$.

Lema 1.10.4. Seja $c: D \subset \mathbb{R}^{2} \rightarrow M$ uma aplicação suave a dois parâmetros. Defina as seguintes curvas: $t \mapsto \gamma_{s}(t):=c(t, s)$ e $s \mapsto \beta_{t}(s):=c(t, s)$. Então

$$
\nabla_{\gamma_{s_{0}}^{\prime}\left(t_{0}\right)}^{V} \beta_{t}^{\prime}\left(s_{0}\right)=\nabla_{\beta_{t_{0}}^{\prime}\left(s_{0}\right)}^{V} \gamma_{s}^{\prime}\left(t_{0}\right)
$$

onde $V$ é um campo suave sem singularidades ao longo de $c(D)$. 
Um campo $X$ ao longo de uma curva regular $\gamma:[a, b] \rightarrow(M, F)$ é dito paralelo quando $D_{\gamma}^{\gamma^{\prime}} X=0$. Dado $v \in T_{\gamma(a)} M \backslash 0$ existe um único campo paralelo $X=X_{v}$ ao longo de $\gamma$ tal que $X(a)=v$. Assim está bem definida a aplicação que associa a cada $(t, v) \in[a, b] \times T_{\gamma(a)} M$ o vetor $X_{v}(t) \in T_{\gamma(t)} M$. Tal aplicação é denominada de transporte paralelo de $v$ ao longo e $\gamma$.

Verifica-se que uma curva $\gamma:[a, b] \rightarrow M$ suave é uma geodésica se, e somente se, seu campo velocidade é paralelo, isto é, $D_{\gamma}^{\gamma^{\prime}} \gamma^{\prime}=0$.

Observação 1.11. Dada F métrica Finsleriana, sejam g e C respectivamente seu tensor fundamental e tensor de Cartan. Para qualquer $c>0$ a aplicação $F_{c}:=c F$ é uma métrica Finsleriana e seu tensor fundamental e de Cartan são dados respectivamente por $g^{c}=c^{2} g$ e $C^{c}=c^{2} C$. Pela equação de Kozul 1.21, $\gamma$ é uma geodésica de F se, e somente se, é uma geodésica de $F_{c}$.

Lema 1.11.1. Se $\gamma:[a, b] \rightarrow M$ é uma geodésica e $X, Y$ são campos ao longo de $\gamma$, então

$$
\frac{d}{d t} g_{\gamma^{\prime}}(X, Y)=g_{\gamma^{\prime}}\left(D_{\gamma}^{\gamma^{\prime}} X, Y\right)+g_{\gamma^{\prime}}\left(X, D_{\gamma}^{\gamma^{\prime}} Y\right)
$$

isto é, a conexão de Chern é g-compatível na direção da geodésica.

Demonstração. Observando que $\frac{d}{d t} g_{\gamma^{\prime}}(X, Y)=\gamma^{\prime} g_{\gamma^{\prime}}(X, Y)$, o resultado segue diretamente da quase compatibilidade da conexão de Chern e da definição de geodésica.

Lema 1.11.2. Seja $(M, F)$ uma variedade Finsleriana. Se $V$ é um campo suave definido em uma aberto $U \subset M$ sem singularidades, então

$$
\nabla_{V}^{V} V=\tilde{\nabla}_{V} V
$$

onde $\tilde{\nabla}$ é conexão Levi-Civita da métrica $\tilde{g}=g_{V}$. Em particular, uma curva regular $\gamma$ : $[a, b] \rightarrow M$ é uma geodésica de $F$ se, e somente se, for uma geodésica da métrica Riemanniana $g_{V}$, para qualquer $V$ extensão local de $\gamma^{\prime}$ sem singularidades.

Demonstração. Pelas fórmulas de Koszul Finsleriana e Riemanniana e pelas propriedades do tensor de Cartan segue que

$$
\begin{aligned}
2 g_{V}\left(\nabla_{V}^{V} V, Z\right) & =V g_{V}(V, Z)-Z g_{V}(V, V)+V g_{V}(Z, V) \\
& +g_{V}([V, V], Z)+g_{V}([Z, V], V)-g_{V}([V, Z], V)=2 g_{V}\left(\tilde{\nabla}_{V} V, Z\right),
\end{aligned}
$$

para qualquer campo suave $Z$ definido em $U$, onde $\tilde{\nabla}$ é a conexão Levi-Civita de $g_{V}$. Isto termina a prova.

\subsubsection{Exponencial e Campos de Jacobi}

Geodésicas são projeções de um campo de vetores em $T M \backslash 0$. Elas dependem suavemente das condições iniciais. Além disso, as geodésicas satisfazem uma condição de homogeneidade. Mais precisamente, se $\gamma_{v}$ é uma geodésica com velocidade $\gamma^{\prime}(0)=v$, então $\gamma_{\lambda v}(t)=\gamma_{v}(\lambda t)$ para qualquer $\lambda>0$. Estes fatos nos permitem definir para qualquer $p \in M$ em uma vizinhança apropriada $U_{p} \subset T_{p} M$ de $0_{p}$ a aplicação exponencial: exp $: U_{p} \backslash 0 \rightarrow M$ com $\exp _{p}(v)=\gamma_{v}(1)$. Tal aplicação é suave em $U_{p} \backslash 0$ e se estende para uma aplicação de classe $C^{1}$ em $U_{p}$. É possível verificar que $d\left(\exp _{p}\right)_{0}$ é a aplicação identidade. 
Dada uma geodésica $\gamma:[a, b] \rightarrow M$ um campo de Jacobi ao longo de $\gamma$ é um campo suave ao longo de $\gamma$ satisfazendo a equação de Jacobi:

$$
J^{\prime \prime}+R_{\gamma^{\prime}}(J)=0,
$$

onde $R_{\gamma^{\prime}}$ é o tensor de Jacobi que é dado por $R_{\gamma^{\prime}}(J):=R_{V}(J):=\nabla_{V}^{V} \nabla_{J}^{V} V-\nabla_{J}^{V} \nabla_{V}^{V} V+$ $\nabla_{[V, J]}^{V} V$ para qualquer extensão $V$ sem singularidades de $\gamma^{\prime}$. Verifica-se que um campo $J$ ao longo de uma geodésica $\gamma$ é um campo de Jacobi se, e somente se, for o campo variacional de uma variação por geodésicas de $\gamma$, vide [15]. Mais precisamente, existe uma aplicação suave a 2-parâmetros $H(t, s) \in M$ tal que fixado $s \gamma_{s}(t):=H(t, s)$ é uma geodésica, $\gamma_{0}=\gamma$ e $J(t)=\left.\frac{\partial}{\partial s}\right|_{s=0} H$.

Proposição 1.11.1 ([25]). Seja $V$ campo geodésico definido em um aberto $U \subset M$. Para qualquer $\gamma$ curva integral de $V$, o tensor $R_{\gamma^{\prime}}$ coincide com o tensor de Jacobi Riemanniano da métrica Riemanniana $\tilde{g}:=g_{V}$. Em particular,

$$
g_{\gamma^{\prime}}\left(X, R_{\gamma^{\prime}}(Y)\right)=g_{\gamma^{\prime}}\left(R_{\gamma^{\prime}}(X), Y\right),
$$

para quaisquer campos $X, Y$ em $U$.

Elementos $p, q \in M$ são ditos pontos conjugados se existe um campo de Jacobi $J$ não trivial ao longo de uma geodésica $\gamma:[a, b] \rightarrow M$ de $p$ a $q$ tal que $J(a)=J(b)=0$.

Lema 1.11.3. Seja $\gamma:[a, b] \rightarrow M$ uma geodésica de $(M, F)$ com $\gamma(a)=p$. Então para quaisquer $u, v \in T_{p} M$ existe um único campo de Jacobi tal que $J(a)=u$ e $J^{\prime}(a)=w$.

Observação 1.12. Dada uma geodésica $\gamma$ em $M^{n}$, pelo Lema 1.11.3, a aplicação que associa a cada $J$ campo de Jacobi ao longo de $\gamma$ o vetor $\left(J(0), J^{\prime}(0)\right) \in T_{p} M \times T_{p} M$ é um isomorfismo linear.

Proposição 1.12.1. Sejam $J_{1}$ e $J_{2}$ campos de Jacobi ao longo de uma geodésica $\gamma$, então $g_{\gamma^{\prime}}\left(J_{1}^{\prime}, J_{2}\right)-g_{\gamma^{\prime}}\left(J_{1}, J_{2}^{\prime}\right)$ é constante.

Demonstração. Pelo Lema 1.11.1, pela definição de campos de Jacobi e pela Proposição 1.11.1 segue que

$$
\begin{aligned}
\frac{d}{d t}\left(g_{\gamma^{\prime}}\left(J_{1}^{\prime}, J_{2}\right)-g_{\gamma}\left(J_{1}, J_{2}^{\prime}\right)\right) & =g_{\gamma^{\prime}}\left(J_{1}^{\prime \prime}, J_{2}\right)-g_{\gamma^{\prime}}\left(J_{1}, J_{2}^{\prime \prime}\right) \\
& =g_{\gamma^{\prime}}\left(R_{\gamma}\left(J_{1}\right), J_{2}\right)-g_{\gamma^{\prime}}\left(J_{1}, R_{\gamma^{\prime}}\left(J_{2}\right)\right) \\
& =g_{\gamma^{\prime}}\left(J_{1}, R_{\gamma}\left(J_{2}\right)\right)-g_{\gamma^{\prime}}\left(J_{1}, R_{\gamma^{\prime}}\left(J_{2}\right)\right)=0 .
\end{aligned}
$$

\subsection{Vizinhanças Tubulares}

Para qualquer ponto $p$ em uma variedade Finsleriana $(M, F)$ existe $U \subset M$ uma vizinhança de $p$ tal que para qualquer $q \in U$ a distância $d(p, q)$ é realizada por uma geodésica partindo de $p$. Em geometria Riemanniana este resultado é generalizado para uma subvariedade, ou seja, dada uma subvariedade fechada $L$ existe $U$ uma vizinhança de $L$ tal que a distância de $L$ a qualquer ponto de $U$ é realizada por uma geodésica ortogonal $L$, vide [19]. Tal vizinhança é denominada de vizinhança tubular. O argumento desta generalização utiliza fortemente a suavidade da aplicação exponencial em torno da seção nula. Desta forma 
tal argumento não pode ser usado no contexto Finsleriano. Pela falta de simetria na métrica, existirão duas vizinhanças tubulares, futura e passada.

Seja $L$ uma subvariedade mergulhada de $(M, F)$ variedade Finsleriana. Definimos a exponencial normal de $L$ como a restrição da exponencial a $\nu(L)$ e a denotaremos por $\exp ^{\nu}: \nu(L) \rightarrow M$.

Definição 1.13.1. Uma vizinhança tubular futura de $L$ com raio $\epsilon$ é um aberto $T u b_{\epsilon}^{+}(L)$ de $M$ contendo $L$ tal que a exponencial normal exp ${ }^{\nu}:=: \nu^{(0, \epsilon)}(L) \rightarrow T u b_{r}^{+}(L) \backslash L$ é um difeomorfismo. A definição de vizinhança tubular passada de raio $\epsilon$, denotada por $T u b_{r}^{-}(L)$ é completamente análoga a de vizinhança tubular futura, considerando apenas a exponencial normal da métrica reversa $\tilde{F}(v):=F(-v)$ no lugar da exponencial normal de $F$.

A existência de vizinhanças tubulares no caso Riemanniano usa fortemente o fato de que a exponencial normal é um difeomorfismo, vide [19]. No contexto Finsleriano em geral a exponencial normal não é suave na seção nula. Então é necessário fazer outro argumento para garantir a existência das vizinhanças tubulares Finslerianas. Será verificada apenas a existência de vizinhança tubular futura, pois a prova para o caso de vizinhança tubular passada é completamente análoga.

Teorema 1.13.1. Seja L uma subvariedade mergulhada em $(M, F)$ variedade Finsleriana. Se $P$ é um aberto pré-compacto de L, então para algum $\epsilon>0$

$$
T u b_{\epsilon}^{+}(P):=\exp \left(\nu^{(0, \epsilon)}(P)\right)
$$

é uma vizinhança tubular futura de $P$.

O primeiro passo na prova é verificar que qualquer $\gamma$ geodésica ortogonal a $L$ minimiza a distância $\rho_{+}(\cdot):=d(L, \cdot)$ para algum intervalo, ou seja, existe $\epsilon>0$ tal que $d(L, \gamma(t))=$ $L\left(\left.\gamma\right|_{(0, t)}\right)$ para qualquer $t \in(0, \epsilon)$. Dizemos que uma geodésica unitária $\gamma:[0, a] \rightarrow M$ é um $L$-segmento quando $\rho_{+}(\gamma(t)):=d_{F}(L, \gamma(t))=t$. Defina o value cut de $L$ em $v=v_{p} \in \nu^{1}(L)$ por

$$
i(v):=i_{L}(v):=\sup \left\{t \in \mathbb{R} ; \rho_{+}\left(\gamma_{v}(t)\right)=t\right\} .
$$

Lema 1.13.1. Se $v, w \in \nu^{1}(L)$ e $t_{0}, t_{1}<\min \{i(v), i(w)\}$ tais que $\gamma_{v}\left(t_{0}\right)=\gamma_{w}\left(t_{1}\right)$, então $v=w$.

Demonstração. Primeiro observe que $t_{0}=t_{1}$, pois do contrário $\gamma_{v}$ ou $\gamma_{w}$ não minimizaria em $t_{0}$ ou $t_{1}$, contradizendo a hipótese. Seja $t_{2} \in\left(t_{0}, i(v)\right)$. Por definição $\rho_{+}\left(\gamma_{v}\left(t_{2}\right)\right)=t_{2}$ e $\rho_{+}\left(\gamma_{w}\left(t_{0}\right)\right)=t_{0}$. Seja $\gamma$ a concatenação de $\left.\gamma_{w}\right|_{\left[0, t_{0}\right]}$ com $\left.\gamma_{v}\right|_{\left[t_{0} . t_{2}\right]}$. Observe que $L(\gamma)=$ $t_{2}=d_{L}\left(\gamma\left(t_{2}\right)\right)$, ou seja, $\gamma$ realiza a distância de $\gamma(0)$ a $\gamma\left(t_{2}\right)$. Portanto $\gamma$ é uma geodésica, implicando que $\gamma_{v}=\gamma_{w}$.

Teorema 1.13.2. Seja L uma subvariedade mergulhada $k$-dimensional em uma variedade Finsleriana $\left(M^{n}, F\right)$. Então dado $v_{0} \in \nu^{1}(L)$ existem $\epsilon>0$ e $\mathcal{U}$ vizinhança aberta de $v_{0}$ em $\nu^{1}(L)$ tais que $i_{L}(v)>\epsilon$ para todo $v \in \mathcal{U}$.

Demonstração. Seja $\varphi: \mathbb{R}^{n} \rightarrow W$ parametrização de $M$ em torno de $p=\pi\left(v_{0}\right)$ tal que $\varphi\left(\mathbb{R}^{k} \times 0\right)=L \cap W$. Considere uma base de $\mathbb{R}^{n}$ da forma $\left\{d \varphi^{-1}\left(v_{0}\right), e_{2}, \ldots, e_{n}\right\}$ e alguma vizinhança $\mathcal{U}$ de $v_{0}$ em $\nu^{1}(L)$ tal que $B_{v}=\left\{d \varphi^{-1}(v), e_{2}, \ldots, e_{n}\right\}$ é uma base de $\mathbb{R}^{n}$ para qualquer $v \in \mathcal{U}$. Defina a métrica $h=\varphi^{*}\left(g_{v}\right)$. Aplicando Gram-Schmidt à base $B_{v}$, obtém-se uma base $h$-ortogonal $\left\{E_{1}(v)=d \varphi^{-1}(v), E_{2}(v), \ldots, E_{n}(v)\right\}$ tal que $E_{i}(v)$ depende suavemente de $v$, para qualquer $i \in\{1, \ldots, n\}$. Defina a aplicação $\phi: \mathbb{R}^{n} \times \mathcal{U} \rightarrow \mathbb{R}^{n}$ por $\phi(x, v)=$ 
$\sum x_{i} E_{i}(v)$, onde $x=\left(x_{1}, \ldots, x_{n}\right)$. Note que $L_{v}:=\varphi \circ \phi\left(\{0\} \times \mathbb{R}^{n-1} \times v\right)$ é uma hipersuperfície contendo $L$ tal que $v$ é ortogonal.

Dado $v \in \mathcal{U}$ o campo unitário normal a $L_{v}$ que estende $v$, denotado por $N_{v}$, depende suavemente de $v$. Esta afirmação segue da equação: $N_{v}(p)=N_{+}\left(\varphi_{v}^{-1}(p), v\right)$, onde $N_{ \pm}$: $\mathbb{R}^{n-1} \times \mathcal{U} \rightarrow T M$ é a aplicação dada por

$$
N_{ \pm}(x, v)=\mathcal{L}^{-1}\left(\left(\varphi_{v}^{-1}\right)^{*} d x_{1(0, x)}\right)
$$

$\operatorname{com} \varphi_{v}(x)=\varphi(\phi(x, v))$ e $\mathcal{L}$ a aplicação de Legendre associada a $F$. No que se segue neste parágrafo, será verificado que $p \mapsto N_{+}\left(\varphi_{v}{ }^{-1}(p), v\right)$ é o campo normal unitário que estende $v$. Como $d x_{1}$ é um covetor unitário com respeito à métrica dual a $h, \varphi_{v}$ é uma isometria e $\mathcal{L}$ preserva a métrica, segue que $N_{ \pm}$são campos unitários com respeito a $F$. Dado $w \in T_{p} L_{v}$, por definição, $w=d \varphi_{v}(0, W)$ e $p=\varphi_{v}(0, x)$ para algum $x, W \in \mathbb{R}^{n-1}$, logo

$$
g_{N_{v}(p)}\left(N_{v}(p), w\right)=\mathcal{L}\left(N_{v}(p)\right)(w)=d x_{1(0, x)}(0, W)=0,
$$

e portanto $N_{v} \in \nu^{1}\left(L_{v}\right)$. Agora note que $d x_{1(0,0)}=h\left(E_{1},.\right)=\varphi^{*} g_{v}\left(d \varphi^{-1}(v),.\right)=\varphi^{*}(\mathcal{L}(v))$, ou seja, $v=\mathcal{L}^{-1}\left(\left(\varphi_{v}{ }^{-1}\right)^{*} d x_{1(0,0)}\right)$.

Defina a aplicação $E: \tilde{W} \subset \mathbb{R} \times \nu^{1}(L \cap W) \times \mathbb{R}^{n-1} \rightarrow \nu^{1}(L \cap W) \times M$

$$
E(s, v, x)=\left(v, \gamma_{N_{v}\left(\varphi_{v}(x)\right)}(s)\right)
$$

onde $\tilde{W}$ é um aberto contendo $\left(0, v_{0}, 0\right)$ tal que $\gamma_{N^{v}(x)}(s)$ está bem definido para qualquer $(s, v, x) \in \tilde{W}$. É preciso verificar que $d E_{\left(0, v_{0}, 0\right)}$ é um isomorfismo linear, ou equivalentemente, $d\left(E_{v_{0}}\right)_{(0,0)}$ é um isomorfismo linear, onde $E_{v}(s, x):=\gamma_{N_{v}(x)}(s)$. Seja $\left\{X_{1}, \ldots, X_{n}\right\}$ base canônica de $\mathbb{R} \times \mathbb{R}^{n-1}$ em torno de $(0,0)$. Logo

$$
\begin{gathered}
d\left(E_{v}\right)_{(0, x)}\left(X_{1}\right)=\left.\frac{d}{d s}\right|_{s=0} E_{v}(s, x)=\gamma_{N_{v}(x)}^{\prime}(0)=N_{v}(x) ; \mathrm{e} \\
d\left(E_{v}\right)_{(0,0)}\left(0, X_{i}\right)=\left.\frac{d}{d s}\right|_{s=0} E_{v}\left(0, s X_{i}\right)=\left.\frac{d}{d s}\right|_{s=0} \varphi_{v}^{-1}\left(s X_{i}\right)=d \varphi_{v}^{-1} X_{i} .
\end{gathered}
$$

E como $\varphi_{v}$ é um difeomorfismo, segue que $d\left(E_{v_{0}}\right)_{(0,0)}$ é um isomorfismo linear.

Pelo Teorema da Função Inversa, existe uma vizinhança aberta $W \subset \tilde{W}$ de $\left(0, v_{0}, 0\right)$ tal que $\left.E\right|_{W}: W \rightarrow E(W)$ é um difeomorfismo. Assuma que $E(W)=\mathcal{U} \times U$ e escolha $\mathcal{U} \times U$ e $\epsilon>0$ tais que $U$ é uma vizinhança convexa de $p_{0}=\pi\left(v_{0}\right)$ e para qualquer $t \in[0, \epsilon]$

1. $\bar{B}_{F}^{-}\left(\gamma_{v}(t), \epsilon\right)$ é um subconjunto compacto de $\mathcal{U}$;

2. $\bar{B}_{F}^{-}\left(\gamma_{v}(t), \epsilon\right) \cap(L \backslash U)=\emptyset ; \mathrm{e}$

3. todas as geodésicas ortogonais a $L_{v}$ de comprimento menor do que $\epsilon$ do lado oposto de $L_{v}$ de $v$ não intersectam $\gamma_{v}([0, \epsilon])$.

Mostraremos que $i_{L}(v)>\epsilon$ para qualquer $v \in \mathcal{U}$. Dado $t \in(0, \epsilon)$ por compacidade existe uma geodésica $\gamma$ que realiza a distância de $\bar{B}_{F}^{-}\left(\gamma_{v}(t), \epsilon\right) \cap L_{v}$ a $\gamma_{v}(t)$. Pelos itens (2) e (3) acima $w=\gamma^{\prime}(0)=r N_{v}(q)$ para algum $r>0$ e $q \in L_{v}$. Pela injetividade a aplicação $E$, temos que $v=w$. Isso implica que $\gamma_{v}:(0, \epsilon) \rightarrow M$ é um $L_{v}$-segmento, logo é um também $L$-segmento, pois $L$ é subvariedade de $L_{v}$. Portanto $i_{L}(v)>\epsilon$. 
Corolário 1.13.1. Seja L uma subvariedade mergulhada em $(M, F)$ variedade Finsleriana. Se $P$ é um aberto pré-compacto de $L$, então existe $\epsilon>0$ tal que $i_{P}(v)>\epsilon$ para qualquer $v \in \nu^{1}(P)$.

Demonstração. Pelo Teorema 1.13.2, existe uma família de pares $\left(\mathcal{U}_{i}, \epsilon_{i}\right)$ onde $\mathcal{U}_{i}$ é uma aberto de $\nu^{1}(L)$ e $\epsilon_{i}>0$ que cumprem a tese do Teorema 1.13 .2 e $\left\{\mathcal{U}_{i}\right\}$ cobre $\{v \in$ $\left.\nu_{p}^{1}(L) ; p \in \bar{P}\right\}$. Como $\left\{v \in \nu_{p}^{1}(L) ; p \in \bar{P}\right\}$ é compacto existe $k \in \mathbb{N}$ tal que $\left\{\mathcal{U}_{i_{1}}, \ldots, \mathcal{U}_{i_{k}}\right\}$ cobre $\nu^{1}(P)$. O número $\epsilon$ definido como o mínimo do conjunto $\left\{\epsilon_{i_{1}}, \ldots, \epsilon_{i_{k}}\right\}$ que cumpre a propriedade desejada.

Proposição 1.13.1. Sejam $P$ uma subvariedade de $(M, F)$ e $\mathcal{U} \subset \nu(P)$ um aberto onde a exponencial está bem definida. Se $v_{0} \in \mathcal{U}$ tal que a geodésica $\gamma_{v_{0}}:[0,1] \rightarrow M$ não contém pontos $P$-focais (vide Definição 4.2), então para alguma vizinhança aberta $\mathcal{U}_{0} \subset \mathcal{U}$ de $v_{0}$ a aplicação exp $: \mathcal{U}_{0} \rightarrow M$ é um difeomorfismo na imagem.

Demonstração. A exponencial normal tem uma singularidade em $v \in \nu(P)$ se, e somente se, existe uma curva suave $w:(-\epsilon, \epsilon) \rightarrow \nu(P)$ tal que $w(0)=v$ e $\left.\frac{\partial}{\partial s}\right|_{s=0} \gamma_{w(s)}(1)=0$. Para verificar esta afirmação basta notar que $d\left(\exp ^{\nu}\right)_{v} w^{\prime}(0)=\left.\frac{\partial}{\partial s}\right|_{s=0} \gamma_{w(s)}(1)=0$. Por outro lado, $J(t)=\left.\frac{\partial}{\partial s}\right|_{s=0} \gamma_{w(s)}(t)$ é um campo $P$-Jacobi. E portanto exp tem uma singularidade em $v$ se, e somente se, $J(1)=0$, e neste caso, $\gamma_{v}(1)$ é um ponto $P$-focal.

Proposição 1.13.2. Sejam $P$ subvariedade mergulhada de $(M, F)$. Assuma que a exponencial esteja bem definida em $v_{0} \in \nu^{1}(P)$. Então existem $\epsilon>0$ e $\mathcal{U}$ vizinhança aberta de $v_{0}$ em $\nu^{1}(P)$ tais que a geodésica $\gamma_{v}:[0, \epsilon] \rightarrow M$ não possui pontos $P$-Jacobi para qualquer $v \in \nu^{1}(P)$.

Demonstração. Primeiro observe que as equações que definem um campo $P$-Jacobi ao longo de $\gamma_{v_{0}}$ é um sistema de Morse-Sturm. Pelo Lema 2.1 em [22], existe $\epsilon>0$ tal que $\gamma_{v_{0}}$ : $[0, \epsilon] \rightarrow M$ não possui ponto $P$-conjugado. Como existe uma dependência suave nas condições iniciais da geodésica, pode-se afirmar a existência de uma vizinhança $\mathcal{U} \subset \nu^{1}(P)$ tal que $\gamma_{v}:[0, \epsilon] \rightarrow M$ não possui ponto $P$-conjugado para qualquer $v \in \mathcal{U}$.

Prova do Teorema 1.13.1. Seja $\epsilon>0$ dado no Corolário 1.13.1. Pelo Lema 2.6.1, para qualquer ponto $p \in \exp ^{\nu}\left(\nu^{(0, \epsilon)}(P)\right)$ existe uma única geodésica unitária normal a $P$ que alcança $p$ realizando a distância $d(P, p)$. Em particular, exp $p^{\nu}$ restrita a $\nu^{(0, \epsilon)}(P)$ é injetora. Por outro lado, pela compacidade e pela Proposição 1.13 .2 o número $\epsilon$ pode ser escolhido de modo que $\gamma_{v}:[0,1] \rightarrow M$ não possua pontos $P$-conjugados para qualquer $v \in \nu^{(0, \epsilon)}(P)$. E portanto $\exp ^{\nu}: \nu^{(0, \epsilon)}(P) \rightarrow M$ é um difeomorfismo local, terminando a demonstração.

\subsection{Submersão Finsleriana}

Nesta seção, com base em [7], serão apresentados alguns resultados envolvendo submersões Finslerianas. Este é o objeto que descreve localmente as folheações Finslerianas regulares. Portanto desempenhará um papel importante neste trabalho.

Uma submersão $\pi:\left(M, F_{1}\right) \rightarrow\left(B, F_{2}\right)$ entre variedades Finslerianas é uma submersão Finsleriana quando o diferencial de $\pi$ aplica a bola futura de $F_{1}$ sobre a bola futura de $F_{2}$, em símbolos, $d \pi\left(B_{p}^{F_{1}}\right)=B_{\pi(p)}^{F_{2}}$, para qualquer $p \in M$, onde $B^{F_{i}}=F_{i}^{-1}[0,1)$.

Seja $\pi:(M, F) \rightarrow(B, Z)$ uma submersão Finsleriana. Então para qualquer $w \in T_{q} B$

$$
Z(w)=\inf \{F(v) ; d \pi(v)=w\} .
$$


Em particular, $Z(d \pi(v)) \leq F(v)$ para qualquer $v \in T M$, vide [7]. Um vetor $v \in T M$ é dito horizontal quando $Z(d \pi(v))=F(v)$. O espaço dos vetores horizontais em $p \in M$ é denominado cone horizontal. Se $F$ é uma métrica Riemanniana, então $\pi$ é uma submersão Riemanniana e o cone horizontal coincide com o subespaço horizontal que é o complemento ortogonal do subespaço vertical e, neste caso, o cone horizontal é uma distribuição suave. Porém, em geral, o cone horizontal em $p \in M$ não é sequer um subespaço vetorial de $T_{p} M$, nem suave na seção nula.

Lema 1.14.1. Se $\pi:(M, F) \rightarrow(B, Z)$ é uma submersão Finsleriana, então o cone horizontal em $p$ coincide com o cone ortogonal a fibra de $\pi$ passando por $p$ adicionando o vetor nulo. Reciprocamente, se $\pi:(M, F) \rightarrow(B, Z)$ é uma submersão tal que

$$
d \pi\left(\nu_{p}^{1}\left(\pi^{-1}(p)\right)\right)=\mathcal{I}_{\pi(p)}^{Z},
$$

para qualquer $p \in M$, então $\pi$ é submersão Finsleriana.

Demonstração. Um vetor $v$ é ortogonal a $\pi^{-1}(\pi(p))$ em $p \in M$ se, e somente se, $t=0$ é ponto crítico da função $f_{u}(t)=F^{2}(v+t u)$, para qualquer $u \in \operatorname{Ker}\left(d \pi_{p}\right)$, pois $\left.\frac{\partial}{\partial t}\right|_{t=0} F^{2}(v+t u)=$ $g_{v}(v, u)$. Pela convexidade da função $F$, isto equivale a dizer que $t=0$ é ponto de mínimo de $f_{u}$ para qualquer $u \in \operatorname{Ker}\left(d \pi_{p}\right)$. Observando que $f_{u}$ é o quadrado da distância da origem ao subespaço afim $v+\mathbb{R} u$, concluímos que $v$ é ortogonal a $\pi^{-1}(\pi(p))$ se, e somente se, $F(v)=\inf \{F(m) ; d \pi(m)=d \pi(v)\}=Z(d \pi(v))$, ou seja, $v$ é horizontal.

Provemos a recíproca. Dado $v \in B_{p}^{F}$, considere $v_{0} \in \nu_{p}\left(\pi^{-1}(p)\right)$ tal que $d \pi(v)=d \pi\left(v_{0}\right)$. Observe que $F\left(v_{0}\right)<F(v)<1$. Além disso, $d \pi(v)=F\left(v_{0}\right) d \pi\left(\frac{v_{0}}{F\left(v_{0}\right)}\right) \operatorname{com} d \pi\left(\frac{v_{0}}{F\left(v_{0}\right)}\right) \in \mathcal{I}_{\pi(p)}^{Z}$, por hipótese. E portanto $d \pi(v) \in B_{\pi(p)}^{Z}$. Dado $y \in B_{\pi(p)}^{Z}$ considere $v_{0} \in \nu_{p}\left(\pi^{-1}(p)\right)$ tal que $d \pi\left(v_{0}\right)=y$. Seja $\lambda>1$ tal que $\lambda y \in \mathcal{I}_{\pi(p)}^{Z}$. Logo $\lambda v_{0} \in \mathcal{I}_{p}^{F}$ e consequentemente $v_{0} \in B_{p}^{F}$.

Proposição 1.14.1. Sejam $\left(V_{1}, F\right)$ um espaço de Minkowski, $\pi: V_{1} \rightarrow V_{2}$ submersão linear e $\varphi$ a restrição de $d \pi$ a $\nu_{0}\left(\pi^{-1}(0)\right)$. Então $F_{2}=F_{1} \circ \varphi^{-1}$ é a única métrica de Minkowski em $V_{2}$ tal que $\pi:\left(V_{1}, F_{1}\right) \rightarrow\left(V_{2}, F_{2}\right)$ é uma submersão Finsleriana.

Demonstração. Seja $B=\left\{\pi(v) \in V_{2} ; F(v) \leq 1\right\}$ e $S$ seu bordo. Observe que $\varphi:=\varphi_{0}$ é um homomorfismo, suave em $\nu_{0}\left(\pi^{-1}(0)\right)$ tal que $\varphi\left(B^{F} \cap \nu_{0}\left(\pi^{-1}(0)\right)\right)=B$, logo $S=$ $\varphi\left(\nu_{0}^{1}\left(\pi^{-1}(0)\right)\right)$.

Considere a aplicação $Z: V_{2} \rightarrow \mathbb{R}$ que associa a cada $v$ o único real positivo tal que $\frac{v}{\lambda} \in S$. Pelo Teorema 1.1.1 se mostrarmos que $S$ é fortemtente convexa temos que $Z$ é uma métrica Finsler. E isso termina a prova, pois por construção $Z^{-1}[0,1)=B=\pi\left(B^{F}\right)$.

Vejamos que $S$ é fortemente convexa. Dada curva suave $\beta:(-\delta, \delta) \rightarrow S$, considere a curva $\alpha:(-\delta, \delta) \rightarrow \nu^{1}\left(\pi^{-1}(0)\right)$ tal que $\pi \circ \alpha=\beta$. Observe que $\varphi$ restrito a $\nu^{1}\left(\pi^{-1}(0)\right)$ é um difeomorfismo em $S$ e que $\beta(0)$ é transversal a $S$. Logo

$$
(\pi \circ \alpha)^{\prime \prime}(0)=\pi\left(\alpha^{\prime \prime}(0)\right)=\pi\left(\nabla_{\alpha^{\prime}(0)}^{0} \alpha^{\prime}(0)\right)+\sigma^{v}\left(\alpha^{\prime}(0), \alpha^{\prime}(0)\right) \beta(0)
$$

onde $\nabla^{0}$ é a conexão canônica em $\nu_{0}^{1}\left(\nu^{-1}(0)\right)$ e $\nabla$ é a conexão canônica em $S$. Isso implica que a segunda forma fundamental de $S$ coincide com a segunda forma fundamental de $\nu_{0}^{1}\left(\pi^{-1}(0)\right)$ que é positiva definida.

Definição 1.14.1. Seja $\pi: M \rightarrow B$ uma submersão. Um campo vetorial $W$ em $M$ é campo projetável quando $d \pi(W)$ é um campo suave em $B$ bem definido, ou equivalentemente $d \pi W_{p}=d \pi W_{q}$ para quaisquer $p, q \in M$ com $\pi(p)=\pi(q)$. O campo suave $W$ é dito básico quando for projetável e F-horizontal. 
Proposição 1.14.2 (Levantamento Horizontal). Sejam $\pi: M^{m} \rightarrow N^{n}$ uma submersão e $F$ uma métrica Finsleriana em $M$. Defina a aplicação $\varphi: \nu(\pi) \rightarrow T N \backslash 0$ como a restrição de $d \pi$ a $\nu(\pi):=\cup_{q \in B} \nu\left(\pi^{-1}(q)\right)$. Então

1. $\varphi$ é uma aplicação 1-homogênea suave que se estende continuamente para a seção nula tal que

$$
\varphi_{p}:=\left.d \pi_{p}\right|_{\nu_{p}\left(\pi^{-1}(q)\right)}: \nu_{p}\left(\pi^{-1}(q)\right) \rightarrow T_{q} N \backslash 0
$$

é um difeomorfismo para quaisquer $q \in N$ e $p \in \pi^{-1}(q)$;

2. se $S$ é uma seção transversal das fibras tal que $\left.\pi\right|_{S}: S \rightarrow N$ é um difeomorfismo, então

$$
\varphi_{S}:=\left.\varphi\right|_{\nu(\pi)_{S}}: \nu(\pi)_{S}:=\{v \in \nu(\pi) ; \pi(v) \in S\} \rightarrow T N \backslash 0
$$

é um difeomorfismo. Em particular, $Z:=\left.\left.F \circ \varphi\right|_{S} ^{-1} \circ d \pi\right|_{T S}$ é uma métrica Finsleriana em $S$ tal que $\varphi_{S}^{-1} \circ d \pi_{x}:\left(T_{x} M, F_{x}\right) \rightarrow\left(T_{x} S, Z_{x}\right)$ é uma submersão Finsleriana para qualquer $x \in S$; $e$

3. $\varphi: \mathcal{B}(\pi) \rightarrow \Gamma(T N)$ é uma bijeção, onde $\mathcal{B}(\pi)$ o espaço dos campos básicos de $\pi$ e $\Gamma(T N)$ o espaço dos campos suaves em $N$.

A aplicação $\varphi^{-1}$ é denominada o levantamento $F$-horizontal de $\pi$.

Demonstração. Primeiro será provado o item 1. A aplicação $\varphi$ é suave, pois é a restrição de uma aplicação suave a uma subvariedade. Tal aplicação é 1-homogênea pela linearidade de $d \pi$ e pela estrutura de cone de $\nu(\pi)$. Definindo $\varphi\left(0_{p}\right)=0_{\pi(p)}$ trivialmente $\varphi$ se estende continuamente para a seção nula.

Neste parágrafo, será verificado que $\varphi_{p}$ é uma bijeção, para quaisquer $q \in N$ e $p \in \pi^{-1}(q)$. A aplicação $d \pi_{p}$ é uma aplicação linear de um espaço de Minkowski em um espaço vetorial, logo, para facilitar a notação, suponha que $\pi:(M, F) \rightarrow N$ é uma submersão linear de um espaço de Minkowski. Neste caso $\pi^{-1}(y)=\pi^{-1}(0)+p$ para quaisquer $y \in N$ e $p \in \pi^{-1}(y)$, por linearidade. Isso implica que para qualquer $v \in \nu_{0}\left(\pi^{-1}(0)\right)$ o subespaço afim $\pi^{-1}(\pi(v))$ é tangente a $\mathcal{I}^{F}(F(v))$ em $v$. Para verificar esta afirmação, note que $v \in \nu_{v}\left(\pi^{-1}(\pi(v))\right)$ e que $T_{v} \mathcal{I}^{F}(F(v))$ coincide com $\left\{w ; g_{v}(v, w)=0\right\}$. Dado $y \in N$ considere o vetor $v$ tal que

$$
d_{F}\left(0, \pi^{-1}(y)\right)=F(v)=r .
$$

Logo $v \in \nu\left(\pi^{-1}(y)\right)$ e consequentemente $v \in \nu_{0}\left(\pi^{-1}(0)\right) \cap \pi^{-1}(y)$. Portanto $\varphi:=\varphi_{0}$ é sobrejetora. Para provar a injetividade considere outro vetor $w \in \nu_{0}\left(\pi^{-1}(0)\right) \cap \pi^{-1}(y)$. Observe que $F(w)=d\left(0, \pi^{-1}(y)\right)=F(v)$. Logo $\pi^{-1}(y)$ é tangente a $\mathcal{I}^{F}(F(v))$ em $w$ e em $v$. Pela convexidade das indicatrizes, $v=w$.

Agora será provado que $\varphi_{p}$ é um difeomorfismo. Como $\nu_{p}\left(\pi^{-1}(q)\right)$ é transversal as fibras de $d \pi$ segue que $d\left(\varphi_{p}\right)_{v}$ é um isomorfismo linear. De fato, seja $X \in T_{v} \nu_{p}\left(\pi^{-1}(q)\right) \cap k e r d\left(\varphi_{p}\right)$, $\log 0$

$$
d(\pi)_{p} X=\frac{d}{d t} \pi \circ \alpha(0)=\frac{d}{d t} \varphi_{p} \circ \alpha(0)=d\left(\varphi_{p}\right) X=0,
$$

onde $\alpha(-\epsilon, \epsilon) \rightarrow \nu_{p}\left(\pi^{-1}(q)\right)$ é uma curva suave que realiza $X$, implicando que $X$ é tangente a fibra, $\operatorname{logo} X=0$, por tranversalidade. E portanto, pelo Teorema da Função Inversa, $\varphi_{p}$ é um difeomorfismo.

Agora será verificado o item 2. Pelo item 1, e pelo Teorema da Aplicação Inversa, para provar o item 2, basta verificar que $T_{v} \nu(\pi)_{S} \cap \operatorname{ker} d \varphi_{S}=0$. Sejam $V$ curva suave em $\nu(\pi)_{S}$ e $W$ curva suave em $T M$ tais que $d \varphi\left(V^{\prime}(0)\right)=0$ e $\varphi W(t)=0$ (ou equivalentemente, $V^{\prime}(0)$ e 
$W$ são tangente as fibras de $\pi)$. Neste caso, a primeira equação implica que $\gamma^{\prime}(0)=0$ onde $\rho(V)=\gamma$. Logo

$$
D_{\gamma}^{V(0)} V(0)=V^{\prime}(0) \text { e } D_{\gamma}^{V(0)} W(0)=W^{\prime}(0),
$$

pela equação 1.18. E como $V$ é uma curva contida nos cones normais às fibras e $W$ é tangente as fibras, temos que

$$
0=g_{V(0)}\left(V^{\prime}(0), W(0)\right)+g_{V(0)}\left(V(0), W^{\prime}(0)\right) .
$$

O campo $W^{\prime}(t)$ também é tangente as fibras, e consequentemente $g_{V(t)}\left(V(t), W^{\prime}(t)\right)=0$. De fato, considerando uma carta folheada da submersão $\varphi=\left(x_{1}, \ldots, x_{k}, x_{k+1}, \ldots, x_{n}\right)$, onde as $k$ primeiras coordenadas são tangentes às fibras, tem-se que

$$
W^{\prime}(t)=\frac{d}{d t}\left(W_{1}(t), \ldots, W_{k}(t), 0, \ldots, 0\right)=\left(W_{1}^{\prime}(t), \ldots, W_{k}^{\prime}(t), 0, \ldots, 0\right),
$$

implicando a afirmação. Portanto $g_{V(0)}\left(V^{\prime}(0), W(0)\right)=0$ e assim pela generalidade da escolha de $W$ o vetor $V^{\prime}(0)$ não tem componente tangente as fibras de $\pi$ e portanto $V^{\prime}(0)=0$.

Por fim será provado o item 3. Se $X$ é projetável, $\varphi(X)$ é um campo suave em $N$, por definição. Reciprocamente, dado $Y$ campo suave em $N, X:=\varphi^{-1}(Y)$ é uma seção do fibrado tangente de $M$ pelo item 1. É necessário verificar a suavidade de $X$. Dado $p \in \pi^{-1}(q) \subset M$ considere $(U, \theta)$ carta local adaptada a $\pi$ em torno de $p$. Suponha que $\theta(U)=V_{1} \times V_{2} \subset$ $\mathbb{R}^{m-n} \times \mathbb{R}^{n}$ tal que $\pi^{-1}\left(V_{1} \times 0\right)=\pi^{-1}(N) \cap U$ e defina $S_{x}=\theta^{-1}\left(\theta(x) \times V_{2}\right)$ uma seção transversal à $\pi$ passando por $x$. Observe que $\left.\pi\right|_{S_{x}}: S_{x} \rightarrow N \cap \pi(U)$ é um difeomorfismo e a dimensão de $S_{x}$ coincide com a dimensão de $N$. Pelo item $2,\left.X\right|_{S_{x}}$ é suave para qualquer $x \in U$. Logo para terminar a prova resta verificar que $\left.X\right|_{\pi^{-1}(y)}$ é uma seção suave do fibrado $\lambda: \nu\left(\pi^{-1}(y)\right) \rightarrow \pi^{-1}(y), \lambda\left(v_{p}\right)=p$, para qualquer $y \in N$, ou equivalentemente, que $\left.X\right|_{\pi^{-1}(y)}$ é uma $(m-n)$-subvariedade de $\nu\left(\pi^{y}\right)$ transversal às fibras de $\lambda$, que coincidem com os cones ortogonais a $\pi^{-1}(y)$. Mas isso segue do Teorema da Função Implícita, pois a aplicação $\left.d \varphi\right|_{\nu\left(\pi^{-1}(y)\right)}: \nu\left(\pi^{-1}(y)\right) \rightarrow T_{y} N \backslash 0$ é uma submersão (pelo item 1) e do fato de que os espaços tangentes as fibras são transversais aos cones.

Lema 1.14.2. Uma submersão $\pi:(M, F) \rightarrow(N, Z)$ é uma submersão Finsleriana se, $e$ somente se, $\varphi_{p}:\left(\nu_{p}\left(\pi^{-1}(q)\right), g_{v}^{F}\right) \rightarrow\left(T_{q} N \backslash 0, g_{\varphi(v)}^{Z}\right)$ é uma isometria Riemanniana para qualquer $v=v_{p} \in \nu(\pi)$.

Demonstração. Suponha que $\pi$ é submersão Finsleriana e que $v \in \nu_{p}\left(\pi^{-1}(q)\right)$. Seja $u \in$ $T_{v} \nu\left(\pi^{-1}(q)\right)$. Se $u=r v$, então

$$
g_{v}^{F}(u, u)=r^{2} g_{v}^{F}(v, v)=r^{2} g_{d \pi v}^{Z}(d \pi v, d \pi v)=g_{\varphi(v)}^{Z}(\varphi(u), \varphi(u)),
$$

por linearidade e por definição de vetor horizontal. Agora suponha que $u$ é tangente a indicatriz $\mathcal{I}^{F}(r)$. Considere $\alpha:(-\epsilon, \epsilon) \rightarrow \nu_{p}\left(\pi^{-1}(q)\right) \cap \mathcal{I}^{F}(r)$ curva suave tal que $\alpha^{\prime}(0)=u$. Observe que $\pi \alpha$ é uma curva suave em $\mathcal{I}^{Z}(r)$. Logo

$$
g_{\alpha}^{F}\left(\alpha, \alpha^{\prime \prime}\right)=0=g_{d \pi \alpha}^{Z}\left(d \pi \alpha, d \pi \alpha^{\prime \prime}\right) .
$$

Por outro lado, usando que $\alpha$ é curva de vetores horizontais, verifica-se que

$$
g_{\alpha}^{F}\left(\alpha^{\prime}, \alpha^{\prime}\right)+g_{\alpha}^{F}\left(\alpha, \alpha^{\prime \prime}\right)=g_{d \pi \alpha}^{Z}\left(d \pi \alpha^{\prime}, d \pi \alpha^{\prime}\right)+g_{d \pi \alpha}^{Z}\left(d \pi \alpha, d \pi \alpha^{\prime \prime}\right) .
$$

Por fim, as equações 1.25 e 1.26, implicam que $g_{v}^{F}(u, u)=g_{\varphi(v)}^{Z}(\varphi(u), \varphi(u))$.

A recíproca segue quase imediatamente usando o Lema 1.14.1 e a hipótese. 
Lema 1.14.3. Se $\pi:(M, F) \rightarrow(N, Z)$ é uma submersão Finsleriana e $V$ um campo $F$ básico sem singularidades definido em um aberto $U \subset M$, então $\pi:\left(M, g_{V}^{F}\right) \rightarrow\left(N, g_{d \pi(V)}^{Z}\right)$ é uma submersão Riemanniana. Em particular, as fibras de $\pi$ constituem uma folheação Riemanniana em $\left(U, g_{V}\right)$.

Demonstração. Pelo Lema 1.14.2, a restrição de $\pi$ a $\nu_{p}(\pi)$ é uma isometria com respeito as métricas Riemannianas $g_{V}$ e $g_{d \pi(V)}$. Logo $d \pi$ restrito a um subespaço $g_{V}$-ortogonal a uma fibra é uma isometria Riemanniana com respeito às métricas $g_{V}$ e $g_{d \pi(V)}$, pelo Lema 1.10.2. Isso implica o resultado.

Seja $\pi:(M, F) \rightarrow(N, Z)$ uma submersão Finsleriana. Uma curva suave $\gamma:[a, b] \rightarrow M$ é dita horizontal se $\gamma^{\prime}(t)$ pertence ao cone horizontal a fibra de $\pi$ passando por $\gamma(t)$, para qualquer $t \in[a, b]$. A curva $\gamma$ é o levantamento horizontal de $\sigma:[a, b] \rightarrow B$ quando $\gamma$ é uma curva horizontal e $\sigma=\pi \circ \gamma$. Dada $\sigma:[a, b] \rightarrow B$ curva suave, para qualquer $p \in M$, existe um único levantamento horizontal $\gamma:[a, b] \rightarrow M$ partindo de $p$, isto é, $\gamma(a)=p$. Se $\gamma:[a, b] \rightarrow M$ é uma curva suave, então o comprimento de $\gamma$ é maior ou igual ao comprimento de $\pi \circ \gamma$; além disso, a igualdade ocorre se, e somente se, $\gamma$ é horizontal.

Proposição 1.14.3. Seja $\pi:(M, F) \rightarrow(B, Z)$ um submersão Finsleriana. Uma curva imersa em $B$ é geodésica se, e somente se, seu levantamento horizontal é uma geodésica em $M$.

Demonstração. Dada curva imersa $\sigma:[0, b] \rightarrow B$, seja $\gamma:[0, b] \rightarrow M$ seu levantamento horizontal. Dado $t_{0} \in[0, b]$, considere $V$ uma extensão local horizontal e sem singularidades de $\left.\gamma^{\prime}\right|_{V}$ definida em uma vizinhança aberta $U$ de $\gamma\left(t_{0}\right)$. Pelo Lema 1.14.3, $\pi:\left(M, g_{V}\right) \rightarrow$ $\left(B, h_{d \pi V}\right)$ é submersão Riemanniana, onde $g$ e $h$ são respectivamente os tensores fundamentais de $F$ e $Z$. Logo $\sigma$ é geodésica de $h_{d \pi V}$ se, e somente se, $\gamma$ é geodésica de $g_{V}$. O resultado segue do Lema 1.11.2.

A translação de uma submersão entre variedades Finslerianas $\pi:(M, F) \rightarrow(N, Z)$ por $W$ campo projetável com $F(W)<1$ é a submersão $\pi:\left(M, F_{W}\right) \rightarrow\left(N, Z_{d \pi(W)}\right)$.

Proposição 1.14.4 (Translação de Submersão Finsleriana). Sejam $\pi:\left(M, F^{1}\right) \rightarrow\left(B, F^{2}\right)$ uma submersão de Finsler e $W$ campo projetável em $M$ tal que $F^{1}(W)<1$. Então a translação de $\pi$ por $W$, isto é, $\pi_{W}:=\pi:\left(M, F_{W}^{1}\right) \rightarrow\left(B, F_{d \pi W}^{2}\right)$ também é uma submersão Finsleriana.

Demonstração. Temos que $F^{2}(d \pi W)<1$, pois $\pi:\left(M, F^{1}\right) \rightarrow\left(B, F^{2}\right)$ uma submersão Finsleriana. Dado $p \in M$, observe que

$$
\begin{gathered}
B_{\left(F_{W}^{1}\right)_{p}}(0,1)=B_{F_{p}^{1}}(0,1)+W \\
B_{\left(F_{d \pi W}^{2}\right)_{p}}(0,1)=B_{F_{p}^{2}}(0,1)+d \pi W .
\end{gathered}
$$

Estas equações, a linearidade de $d \pi$ e a definição de submersão Finsleriana implicam que $d \pi\left(B_{\left(F_{W}^{1}\right)_{p}}(0,1)\right)=B_{\left(F_{d \pi W}^{2}\right)_{p}}(0,1)$. E portanto $\pi:\left(M, F_{\widetilde{W}}^{1}\right) \rightarrow\left(B, F_{W}^{2}\right)$ é uma submersão Finsleriana.

\subsection{Teorema de Wang caso Finsler}

Nesta seção será apresentado uma generalização do Teorema A em [27] para o contexto Finsleriano. Esta generalização foi demonstrada em [14], porém em breve a tese de Hengameh Raeisi fornecerá uma prova alternativa e outros teoremas sobre funções transnormais Finslerianas. Tal generalização será utilizada na demonstração do Teorema do Slice 2.0.2. 
Considere uma função suave $f$ sobre uma variedade Finsleriana $(M, F)$ e $U=U_{f}$ o conjunto dos valores regulares de $f$. O F-gradiente de $f$, denotado por $\nabla f$, é o campo vetorial definido no aberto $U$ por

$$
g_{\nabla f}(\nabla f, \cdot)=d f(\cdot)
$$

Estendemos $\nabla f$ para $M$ definindo $\nabla f(q)=0$, se $q \notin U_{f}$. O gradiente é suave em $U_{f}$ e contínuo em $M \backslash U_{f}$. Quando não houver risco de confusão diremos apenas que $\nabla f$ é o gradiente de $f$.

Lema 1.15.1. O gradiente de uma função suave em uma variedade Finsler está no cone ortogonal aos conjuntos de níveis regulares. Além disso, função cresce na direção do gradiente.

Demonstração. Dada uma curva suave $\gamma:(-\epsilon, \epsilon) \rightarrow f^{-1}(c)$, temos que

$$
0=\left.\frac{d}{d t}\right|_{t=0} f \circ \gamma=d f\left(\gamma^{\prime}(0)\right)=g_{\nabla f}\left(\nabla f, \gamma^{\prime}(0)\right) \text {. }
$$

Implicando que o gradiente é ortogonal a $f^{-1}(c)$, pela generalidade da curva.

Para provar que $f$ cresce na direção do gradiente, basta considerar $\gamma$ curva integral de $\nabla f$ e observar que $\frac{d}{d t} f \circ \gamma(t)=F^{2}(\nabla f)>0$.

Observe que se o intervalo $[c, d]$ está contido no conjunto regular de $f$, então qualquer curva integral do gradiente partindo de algum ponto de $f^{-1}(c)$ segue por $f^{-1}(c, d)$ até alcançar $f^{-1}(d)$.

Lema 1.15.2. Sejam $f$ função suave em $M$ e $\nabla f$ seu F-gradiente. Defina em $U_{f}$ a métrica Riemanniana $\tilde{g}=g_{\nabla f}$. Então

$$
\nabla f=\tilde{\nabla} f
$$

onde $\tilde{\nabla} f \tilde{g}$-gradiente de $f$. Além disso,

$$
F(\nabla f)=\|\tilde{\nabla} f\|
$$

onde $\|\|=.\sqrt{\tilde{g}}$.

Demonstração. Dado $v \in T_{p} M$, por definição

$$
\tilde{g}(\tilde{\nabla} f, v)=d f(v)=g_{\nabla f}(\nabla f, v)=\tilde{g}(\nabla f, v),
$$

portanto $\nabla f=\tilde{\nabla} f$. Para verificar a segunda equação basta considerar $v=\nabla f$.

Proposição 1.15.1. Seja $Z=h_{W}$ métrica Randers sobre $M$. Dada função suave $f: M \rightarrow$ $\mathbb{R}$, denote por $\tilde{\nabla} f$ e $\nabla f$ respectivamente o h-gradiente e $Z$-gradiente de $f$. Logo

$$
\begin{aligned}
\tilde{\nabla} f & =\frac{Z(\nabla f)}{\lambda Z(\nabla f)+h(\nabla f, W)}(\nabla f-Z(\nabla f) W) e \\
\nabla f & =\left(1+h\left(\frac{\tilde{\nabla} f}{\|\tilde{\nabla} f\|}, W\right)\right)(\tilde{\nabla} f+\|\tilde{\nabla} f\| W),
\end{aligned}
$$

onde $\lambda=1-h(W, W)$ e $\|\|=.\sqrt{h}$. 
Demonstração. Pelo Lema 1.7.1 e pela hipótese de que $\nabla f$ é o $Z$-gradiente de $f$

$$
\frac{Z(\nabla f)}{\lambda \alpha(\nabla f)} h(\nabla f-Z(\nabla f) W, v)=g_{\nabla f}(\nabla f, v)=d f(v)
$$

implicando a primeira equação da proposição, pois $\lambda \alpha(\cdot)=\lambda Z(\cdot)+h(\cdot, W)$.

Provemos a segunda equação da proposição. Denote por $\tilde{v}=(\tilde{\nabla} f+\|\tilde{\nabla} f\| W)$ e observe que $Z(\tilde{v})=\|\tilde{\nabla} f\|$. Agora note que

$$
k:=1+h\left(\frac{\tilde{\nabla} f}{\|\tilde{\nabla} f\|}, W\right)=\frac{\lambda\|\tilde{\nabla} f\|+h(\tilde{v}, W)}{\|\tilde{\nabla} f\|}=\frac{\lambda \alpha(\tilde{v})}{Z(\tilde{v})}>0 .
$$

Logo, pelo Lema 1.7.1 e pela hipótese de que $\tilde{\nabla} f$ é o $h$-gradiente de $f$, temos que

$$
\begin{aligned}
g_{k \tilde{v}}(k \tilde{v}, u) & =k g_{\tilde{v}}(\tilde{v}, u)=k \frac{Z(\tilde{v})}{\lambda \alpha(\tilde{v})} h(\tilde{v}-Z(\tilde{v}) W, u) \\
& =h(\tilde{\nabla} f, u)=d f(u),
\end{aligned}
$$

para qualquer $u \in T M$.

Definição 1.15.1. Uma função $f: M \rightarrow \mathbb{R}$ sobre uma variedade Finsler $(M, F)$ é $F$ transnormal se existir uma função $b: f(M) \rightarrow \mathbb{R}$ de classe $C^{2}$ tal que

$$
F^{2}(\nabla f)=b \circ f .
$$

Exemplo 1.15.1. Por definição, qualquer função distância em uma variedade Finsler $(M, F)$, que seja suave fora da pré-imagem do zero, é F-transnormal, (ver [25], Capítulo 3).

O Teorema de Wang (Teorema A em [27]) não pode ser generalizado integralmente para o caso Finsler. Como contra-exemplo basta considerar a funçao distância à origem definida em um espaço Randers-Minkowski não-reversível. Porém é possível obter a seguinte generalização parcial, conforme [14].

Teorema 1.15.1. Seja $f:(M, F) \rightarrow \mathbb{R}$ função F-transnormal $\left(F^{2}(\nabla f)=b \circ f\right)$ em $(M, F)$ variedade Finsleriana conexa e completa para frente. Se $c, d \in f(M)$ tais que $c<d$, então

$$
d_{F}\left(f^{-1}(c), p\right)=d_{F}\left(f^{-1}(c), q\right)=\int_{c}^{d} \frac{d s}{\sqrt{b(s)}},
$$

para qualquer $p, q \in f^{-1}(d)$. Além disso, esta distância é realizada por qualquer curva integral do gradiente (que é uma geodésica ortogonal aos niveis regulares) partindo de $f^{-1}(c) e$ alcançando $f^{-1}(d)$. Em particular, $f^{-1}(c)$ é paralelo a $f^{-1}(d)$. 


\section{Capítulo 2}

\section{Folheações Finslerianas Singulares, Definições e Propriedades}

Folheações Finslerianas regulares são folheações descritas localmente por submersões Finslerianas satisfazendo uma condição de cociclo. Já folheação Finsleriana singular é uma folheação singular que cumpre a condição de transnormalidade, ou seja, qualquer geodésica que parte ortognonal a uma folha é ortogonal a qualquer folha que encontrar. Verifica-se que estas definições coincidem em se tratando de uma folheação singular com folhas de mesma dimensão. Além de definir tais objetos, serão generalizadas algumas propriedades das folheações Riemannianas singulares para o contexto Finsleriano, obtendo a estrutura infinitesimal. Como referência para o estudo das folheações Riemannianas singulares veja [1], [3], [5], [6], [21].

A primeira propriedade a ser estendida é a estrutura tubular (Proposição 2.6.1). Uma folheação singular é Finsleriana se, e somente se, localmente suas folhas estão contidas em cilindros geométricos futuro e passado tendo como eixo uma placa fixada. Consequentemente, caracteriza-se estes objetos como folheações singulares cujas as folhas são localmente equidistantes; e também prova-se o Lema de Homotetia (Lema 2.6.2). Este lema é um instrumento poderoso que permite concluir propriedades importantes para esta teoria e para a demonstração dos nossos resultados, por exemplo, propriedades de Estratificação e o Teorema de Slice.

O conjunto formado pela união das folhas de uma folheação Finsleriana singular de mesma dimensão possui estrutura de variedade (Proposição 2.7.3), desta forma é obtida uma estratificação da variedade. Além do mais o resultado abaixo permitirá reduzir o Teorema 1 ao caso regular.

Proposição 2.0.2. Sejam $(M, F)$ variedade Finsleriana completa e $(M, F, \mathcal{F})$ folheação Finsleriana singular. Se hé uma métrica Riemanniana completa em $M$ tal que $\left(\Sigma,\left.h\right|_{\Sigma},\left.\mathcal{F}\right|_{\Sigma}\right)$ é folheação Riemanniana para qualquer estrato $\Sigma$, então $(M, h, \mathcal{F})$ é folheação Riemanniana singular.

O Teorema de Slice possibilita o estudo de uma folheação Finsleriana singular do ponto de vista infinitesimal, relacionando-a com uma folheação Finsleriana singular em um espaço de Minkowski. Isso será fundamental na demonstração do teorema principal.

Teorema 2.0.2 (Slice). Seja $(M, F, \mathcal{F})$ uma folheação Finsleriana singular. Então para qualquer $q \in M$ existe um slice $S=S_{q}$ passando por $q$ e uma métrica Finsleriana $Z_{S}$ em $S$ tal que a folheação slice $\mathcal{F}_{q}:=\{L \cap S\}$ é uma folheação Finsleriana singular em $\left(S, Z_{S}\right)$ tal que q é uma folha 0 -dimensional e

$$
d_{F}\left(P_{p}, P_{z}\right)=d_{Z_{S}}\left(P_{p} \cap S, P_{z} \cap S\right)
$$


para quaisquer placas $P_{p}$ e $P_{z}$ de $(M, F, \mathcal{F})$ com $p, z \in S$. Além disso, a folheação slice $\mathcal{F}_{q}$ é difeomorfa a uma folheação Finsleriana singular em um espaço de Minkowski.

Na seção 2.1 será definida folheação Finsleriana regular e provada a Conjectura 1 enunciada na introdução para o caso regular. Na seção 2.2 definiremos folheação singular, estabelecendo algumas propriedades básicas, e depois definiremos folheações Finslerianas singulares, expondo alguns exemplos. Na última seção serão desenvolvidas as propriedades de folheação Finsleriana singular citadas acima.

\subsection{Folheação Finsleriana Regular}

Um cociclo folheado $k$-dimensional (cociclo de Haefliger) é uma família $\left\{U_{i}, s_{i}, \gamma_{i j}\right\}$, onde $\left\{U_{i}\right\}$ é uma cobertura por abertos de $M,\left\{s_{i}: U_{i} \rightarrow \mathbb{R}^{n-k}\right\}$ e $\left\{\gamma_{i j}: s_{j}\left(U_{i} \cap U_{j}\right) \rightarrow s_{i}\left(U_{i} \cap U_{j}\right)\right\}$ são famílias de submersões e difeomorfismos, respectivamente, que atendem a condição de cociclo, isto é,

$$
\left.\gamma_{i j} \circ s_{j}\right|_{U_{i} \cap U_{j}}=\left.s_{i}\right|_{U_{i} \cap U_{j}} .
$$

As fibras das submersões do cociclo folheado se juntam e formam uma partição $\mathcal{F}$ de $M$ por subvariedades imersas chamadas de folhas. O par $(M, \mathcal{F})$ também é chamado de folheação k-dimensional.

Exemplo 2.1.1. As órbitas da ação $\mu: S^{1} \times S^{3} \rightarrow S^{3} \subset \mathbb{C}^{2}$ dada por $\left(e^{i t},\left(z_{1}, z_{2}\right)\right) \mapsto$ $e^{i t}\left(z_{1}, z_{2}\right)$ constituem uma folheação unidimensional de $S^{3}$.

Definição 2.1.1. Um campo projetável de uma folheação regular $(M, \mathcal{F})$ é um campo suave em $M$ que é projetável com respeito a todas as submersões do cociclo de $\mathcal{F}$.

Definição 2.1.2. Uma folheação Finsleriana (Riemanniana) é a tripla $(M, F, \mathcal{F}$ ) onde $(M, F)$ é variedade Finsleriana (Riemanniana) e $\left(M^{n}, \mathcal{F}^{k}\right)$ é folheação regular tal que, para alguma família de métricas Finslerianas (Riemannianas) $\left\{F_{i}\right\}$, seu cociclo de Haefliger $\left\{U_{i}, s_{i}, \gamma_{i j}\right\}$ satisfaz as seguintes propriedades

1. $s_{i}:\left(U_{i},\left.F\right|_{U_{i}}\right) \rightarrow\left(\mathbb{R}^{n-k}, F_{i}\right)$ é uma submersão Finsleriana (Riemanniana);

2. $\gamma_{i j}:\left(s_{i}\left(U_{i} \cap U_{j}\right), F_{i}\right) \rightarrow\left(s_{j}\left(U_{i} \cap U_{j}\right), F_{j}\right)$ é uma isometria Finsleriana (Riemanniana).

Teorema 2.1.1 ([18]). Se $(M, F, \mathcal{F})$ é uma folheação Finsleriana, então existe g métrica Riemanniana tal que $(M, g, \mathcal{F})$ é folheação Riemanniana.

Demonstração. Seja $\left(U_{i}, s_{i}, \gamma_{i j}\right)$ um cociclo de Haefliger de $(M, F, \mathcal{F})$. Para cada $i$ defina em $U_{i}$ e em $s_{i}\left(U_{i}\right)$ respectivamente as métricas Riemannianas dadas pelas médias:

$$
\begin{aligned}
g_{p}^{i}(u, w) & =\frac{1}{\operatorname{vol}_{i}\left(\mathcal{I}_{p}^{F} \cap \nu_{p}\left(L_{p}\right)\right)} \int_{\mathcal{I}_{p}^{F} \cap \nu_{p}\left(L_{p}\right)} g_{v}^{F}(u, w) d v_{i} \\
h_{q}^{i}(x, y) & =\frac{1}{\operatorname{vol}_{i}\left(\mathcal{I}_{q}^{Z_{i}}\right)} \int_{\mathcal{I}_{q}^{Z_{i}}} g_{z}^{Z_{i}}(x, y) d z_{i},
\end{aligned}
$$

onde $d v_{i}=s_{i}^{*}\left(d z_{i}\right), d z_{i}$ é a medida de Hausdorff em $\mathcal{I}_{q}^{Z_{i}}$ e $Z_{i}$ é uma métrica Finsler em $s_{i}\left(U_{i}\right)$ tal que $s_{i}$ é submersão Finsleriana. 
Verifiquemos que $s_{i}:\left(U_{i}, g^{i}\right) \rightarrow\left(s_{i}\left(U_{i}\right), h^{i}\right)$ é uma submersão Riemanniana. Como $s_{i}$ é submersão Finsleriana, $\left(d s_{i}\right)_{p}$ restrito a $\mathcal{I}_{p}^{F} \cap \nu_{p}\left(L_{p}\right)$ é um difeomorfismo em $\mathcal{I}_{\pi(p)}^{Z_{i}}$ e $g_{v}^{F}(u, w)=$ $g_{d s_{i}(v)}^{Z_{i}}\left(d s_{i}(u), d s_{i}(w)\right)$. Logo por mudança de variável

$$
\begin{aligned}
s_{i}^{*}\left(h^{i}\right)_{p}(u, w) & =\frac{1}{\operatorname{vol}_{i}\left(\mathcal{I}_{q}^{Z_{i}}\right)} \int_{\pi_{*}\left(\mathcal{I}_{p}^{F} \cap \nu\left(L_{p}\right)\right)} g_{z}^{Z_{i}}\left(d s_{i}(u), d s_{i}(w)\right) d z_{i} \\
& =\frac{1}{\operatorname{vol}_{i}\left(\mathcal{I}_{q}^{Z_{i}}\right)} \int_{\mathcal{I}_{p}^{F \cap \nu\left(L_{p}\right)}} g_{d s_{i}(v)}^{Z_{i}}\left(d s_{i}(u), d s_{i}(w)\right) s_{i}^{*}\left(d z_{i}\right) \\
& =\frac{1}{\operatorname{vol}_{i}\left(\mathcal{I}_{p}^{F} \cap \nu_{p}\left(L_{p}\right)\right)} \int_{\mathcal{I}_{p}^{F} \cap \nu\left(L_{p}\right)} g_{v}^{F}(u, w) d v_{i}=g_{p}^{i}(u, w) .
\end{aligned}
$$

Analogamente provamos que a aplicação $\gamma_{i j}:\left(s_{i}\left(U_{i} \cap U_{j}\right), h^{i}\right) \rightarrow\left(s_{j}\left(U_{i} \cap U_{j}\right), h^{j}\right)$ é uma isometria Riemanniana que para qualquer $i, j$.

Seja $g$ a métrica Riemanniana em $M$ definida para cada $p \in M$ como o produto interno $g_{p}=g_{p}^{i}$ para algum $i$ tal que $p \in U_{i}$. Vejamos que está bem definida esta métrica. Dados $i, j$, sabemos que

$$
\begin{aligned}
\operatorname{vol}\left(\mathcal{I}_{p}^{F_{i}} \cap \nu_{p}\left(L_{p}\right)\right) & =\operatorname{vol}\left(\mathcal{I}^{Z_{i}}\right)=\left|\operatorname{det}\left(\gamma_{i j}\right)\right| \operatorname{vol}\left(\mathcal{I}^{Z_{j}}\right) \\
& =\left|\operatorname{det}\left(d \gamma_{i j}\right)\right| \operatorname{vol}\left(\mathcal{I}^{Z_{j}}\right)=\left|\operatorname{det}\left(d \gamma_{i j}\right)\right| \operatorname{vol}\left(\mathcal{I}^{F_{j}} \cap \nu\left(L_{p}\right)\right) .
\end{aligned}
$$

Por outro lado, $d v_{i}=\left|\operatorname{det}\left(d \gamma_{i j}\right)\right| d v_{j}$ e portanto $g_{p}^{i}=g_{p}^{j}$ para qualquer $p \in U_{i} \cap U_{j}$.

\subsection{Folheação Finsleriana Singular}

Seja $\mathcal{F}$ uma partição por subvariedades imersas em uma variedade suave $M$. O par $(M, \mathcal{F})$ é uma folheação singular quando para qualquer $v \in T_{p} L_{p}$ existe um campo $X$ definido em $U$ uma vizinhança aberta de $p$ tal que $X(p)=v$ e $X(q) \in T_{q} L_{q}$ para qualquer $q \in U$. As subvariedades $L_{p}$ são chamadas de folhas e a dimensão de $\mathcal{F}$ é o máximo das dimensões das folhas de $\mathcal{F}$. Uma folha de dimensão máxima é chamada de folha regular, caso contrário, é chamada de folha singular. Um ponto é chamado de regular se está contido em uma folha regular e caso contrário de ponto singular.

Verifica-se facilmente que uma folheação singular possui as folhas com mesma dimensão se, e somente se, é uma folheação regular, isto é, gerada por submersões.

Lema 2.2.1. Sejam $\mathcal{F}_{1}=\left\{L^{1}\right\}$ e $\mathcal{F}_{2}=\left\{L^{2}\right\}$ folheações singulares sobre uma variedade $M$. Se $T_{p} L_{p}^{1} \subset T_{p} L_{p}^{2}$ para todo $p \in M$, então $\mathcal{F}_{1}$ é uma subfolheação de $\mathcal{F}_{2}$, isto é, $L_{p}^{1} \subset L_{p}^{2}$ para qualquer $p \in M$.

Demonstração. Suponha, sem perda de generalidade, que $L_{p}^{1}$ é conexo. Dado $q \in L_{p}^{1}$ seja $\gamma:[0,1] \rightarrow L_{p}^{1}$ curva suave ligando $p$ a $q$. Logo, da hipótese, segue que $\gamma^{\prime} \in T_{\gamma} L_{p}^{2}$ e portanto $\gamma \subset L_{p}^{2}$ terminando a prova.

Definição 2.2.1. Seja $(M, \mathcal{F})$ uma folheação singular. Uma placa de $\mathcal{F}$ passando por $q \in M$ é uma vizinhança aberta de $q$ em $L_{q}$ conexa e pré-compacta, que será denotada por $P_{q}$.

Lema 2.2.2. Se $(M, \mathcal{F}=\{L\})$ é folheação singular, então para qualquer $q \in M$ existem $U$, $S=S_{q}$ e $\left(U, \mathcal{F}^{2}=\left\{L^{2}\right\}\right)$, respectivamente, uma vizinhança aberta de $q$, uma subvariedade mergulhada $S=S_{q}$, chamada de slice, e uma folheação regular tais que 
1. $\mathcal{F}^{2}$ é uma subfolheação de $\left.\mathcal{F}\right|_{U}$;

2. $L_{q}^{2}$ é uma placa de $L_{q}$ passando por q; e

Em particular, se $\mathcal{F}$ é regular, para qualquer $p \in U$ a folha $L_{p}^{2}$ coincide com a placa de $\mathcal{F}$ em $U$ passando por $p$.

Demonstração. Sejam $X_{1}, \ldots, X_{k}$ campos folheados de $\mathcal{F}$ definidos em uma vizinhança aberta $U$ de $q$ tais que $\left\{X_{i}(q)\right\}$ é uma base para $T_{q} L_{q}$. Considere $S$ uma subvariedade transversal à $L_{q}$ por $q$ tal que $T_{q} M=T_{q} S \oplus T_{q} L_{q}$ (por exemplo, $S=\left\{\exp _{q}(v) ; h(v, v)<r\right.$ e $h(v, w)=$ $\left.0, \forall w \in T_{q} L_{q}\right\}$ para qualquer métrica Riemanniana $h$ e um $r$ suficientemente pequeno). Diminuindo $S$, é possível definir a aplicação $\varphi:(-\epsilon, \epsilon)^{k} \times S \rightarrow M$, dada por $\varphi\left(t_{1}, \ldots, t_{k}, y\right)=$ $\varphi_{t_{k}}^{k} \circ \ldots \circ \varphi_{t_{1}}^{1}(y)$, sendo $\varphi^{i}$ é o fluxo de $X_{i}$. Diminuindo $U$ se necessário, a aplicação $\varphi$ é um difeomorfismo em $U$. Esta aplicação define uma folheação regular de $U$, denotada por $\mathcal{F}^{2}$, cujas folhas são da forma $\varphi\left((-\epsilon, \epsilon)^{k}, y\right)$.

Dada placa $P$ (k-dimensional) de uma folheação singular $\mathcal{F}$ em $\left(M^{n+k}, F\right)$ e $U:=$ $T u b_{r}^{+}(P) \backslash P$, defina $\Pi: U \rightarrow P$ como a aplicação que associa a cada $x \in U$ o único $p \in P$ tal que $d(P, x)=d(p, x)$, ou equivalentemente $\Pi(x)=\rho \circ\left(\exp ^{\nu}\right)^{-1}(x)$, onde $\exp ^{\nu}$ : $\nu^{(0, r)}(P) \rightarrow T u b_{r}^{+}$é a exponencial normal e $\rho: T M \rightarrow M$ é a projeção canônica. Observe que $\Pi$ é uma submersão, pois é uma composição de uma submersão com um difeomorfismo.

Dado $y \in U$ defina $\Lambda_{y}$ o slice Finsleriano passando por $y$ como a fibra de $\Pi$ contendo $y$ $\left(\Lambda_{y}:=\Pi^{-1}(\Pi(y))\right)$, que é uma subvariedade $n$-dimensional e coincide com $\exp _{\Pi(y)}\left(\nu^{(0, r)}(P)\right)$.

Lema 2.2.3. O espaço tangente a um slice Finsleriano é dado por

$$
T_{y} \Lambda_{y}=\operatorname{Ker}\left(d \Pi_{y}\right)=d\left(\exp _{\Pi(y)}\right)_{v}\left(\sigma^{v}\right)
$$

onde $v=\left(\exp ^{\nu}\right)^{-1}(y)$ e $\sigma^{v}:=\mathcal{L}_{v}^{-1}(0)=\left\{X \in T_{\rho(v)} M ; g_{v}\left(X, Y_{i}(\rho(v))\right)=0\right\}$.

Demonstração. A primeira igualdade segue por definição. No que se segue será verificada a segunda igualde. Pela definição de $\Lambda_{y}$ basta provar que $T_{v} \nu(P)=\sigma^{v}$, onde $v=\left(\exp ^{\nu}\right)^{-1}(y)$. Seja $\alpha \subset \nu(L)$ curva suave com $\alpha(0)=v$. Dado $y \in T_{q} L$ seja $Y$ campo em $T_{q} M$ ao longo de $\alpha$ paralelo com respeito a $g_{\alpha}$ tal que $Y(0)=y$. Por definição $g_{\alpha}(\alpha, Y)=0$ e portanto

$$
0=\frac{d}{d t} g_{\alpha}(\alpha, Y)=g_{\alpha(0)}\left(\alpha^{\prime}(0), Y\right)+g_{\alpha(0)}\left(\alpha(0), Y^{\prime}(0)\right)+C_{\alpha(0)}\left(\nabla_{\alpha}^{\alpha} \alpha, \alpha, Y\right)=g_{v}\left(\alpha^{\prime}(0), Y\right) .
$$

Isso implica que $T_{v} \nu(P) \subset \sigma^{v}$, terminando a prova, pois as dimensões destes espaços coincide.

Lema 2.2.4. Sejam $\mathcal{F}$ uma folheação singular em uma variedade Finsleriana $(M, F)$ e $P$ uma placa. Então existe $U$ vizinhança tubular de $P$ tal que $T_{y} M=T_{y} P_{y}+T_{y} \Lambda_{y}$, para qualquer placa $P_{y} \subset U$.

Demonstração. Da paracompacidade das placas, basta mostrar que as folhas da subfolheação regular $\mathcal{F}^{2}$ são transversais aos slices Finslerianos para uma vizinhança de um ponto $p \in P$.

Considere uma vizinhança aberta $U$ de $p$ em $M$ onde está definido um referencial local $\left\{X_{1}, \ldots, X_{n}, Y_{1}, \ldots, Y_{m}\right\}$ tal que $\left\{Y_{1}(x), \ldots, Y_{m}(x)\right\}$ é base de $T_{x} L^{2}$ para qualquer $x \in U$. Para cada $v \in \nu^{1}(P \cap U)$ e $t \in(0, r)$, defina

$$
Z_{i}^{v}(t)=d\left(\exp _{\rho(v)}\right)_{t v}\left(X_{i}^{v}\right)
$$


onde $X_{i}^{v}$ é a projeção $g_{v}$-ortogonal de $X_{i}(\rho(v))$ sobre $\sigma^{v}$. Note que por construção e pelo Lema 2.2.3, $Z_{i}^{v}(t) \in T_{\exp (t v)} \Lambda$. E como $\left\{X_{i}^{v}\right\}$ é um conjunto linearmente independente, existe $\epsilon_{0}$ tal que $\left\{Z_{i}^{v}(t)\right\}$ é uma base de $T_{\exp (t v)} \Lambda$ para qualquer $v \in \nu^{1}(P \cap U)$ e $t \in\left(0, \epsilon_{0}\right)$. Logo, por continuidade e compacidade, existe $\epsilon$ tal que $\left\{Z_{i}^{v}(t), Y_{i}\left(\exp _{\rho(v)}(t v)\right)\right\}$ é base de $T_{\exp (t v)} M$, para qualquer $(t, v) \in(0, \epsilon) \times \nu^{1}(P \cap U)$, pois $\left\{X_{i}^{v}, Y_{j}(\rho(v))\right\}$ é base de $T_{\rho(v)} M$ para qualquer $v$. Portanto a restrição de $\mathcal{F}^{2}$ ao aberto $\{\exp (t v) ;(t, v) \in(0, \epsilon) \times U\}$ é transversal à $\Lambda_{\rho(v)}$.

Observação 2.3. O Lema 2.2.4 implica que para qualquer placa $P_{x} \subset U$ a aplicação $\pi$ : $P_{x} \rightarrow P$ definida como a restrição $\pi:=\left.\Pi\right|_{P_{x}}$ é uma submersão. Isso porque se $\Pi: U \rightarrow P$ é uma submersão e $L$ uma subvariedade de $U$ tais que $T_{y} U=T_{y} L+T_{y} \Pi^{-1}(\Pi(y))$, então $\left.\Pi\right|_{L}$ é uma submersão. De fato, dado $Y \in T P$, existe $X \in T_{y} U$ tal que $d \Pi(X)=Y$, pois $\Pi$ é submersão. Por hipótese, $X=X_{1}+X_{2} \operatorname{com} X_{1} \in T_{y} L$ e $X_{2} \in T_{y} \Pi^{-1}(\Pi(y))$. Logo $d \Pi\left(X_{1}\right)=Y$, pois $T_{y} \Pi^{-1}(\Pi(y))=\operatorname{Ker}\left(d \Pi_{y}\right)$. Portanto $\left.\Pi\right|_{L}$ é uma submersão.

Observação 2.4. Uma partição de uma variedade $M$ por subvariedades mergulhadas não necessariamente constitui uma folheação singular. De fato, considere em $\mathbb{R}^{3}$ a seguinte partição: dado $p=\left(p_{1}, p_{1}, p_{3}\right)$, se $p_{1}=p_{2}=0$, defina $L_{p}=\mathbb{R}(0,0,1)$ e, caso contrário, $L_{p}=S^{2}\left(0, p_{1}^{2}+p_{2}^{2}\right) \times\left\{p_{3}\right\}$, isto é, o círculo contido no plano $z=p_{3}$ com centro em $\left(0,0, p_{3}\right) e$ raio $p_{1}^{2}+p_{2}^{2}$; esta é uma partição de $\mathbb{R}^{3}$ por subvariedades mergulhadas, porém o vetor $(0,0,1)$ não pode ser estendido para um campo contínuo que é tangente a estas subvariedades.

Definição 2.4.1. Sejam $(M, \mathcal{F})$ uma folheação singular e $W$ campo suave em $M$, seja $\lambda: \mathcal{U}=\cup_{p \in M} U_{p} \rightarrow M,(t, q) \rightarrow \lambda_{t}(q)$ seu fluxo. Dizemos $W$ é folheado quando para qualquer $p \in M$ o difeomorfismo local $\lambda_{t}: U_{p} \rightarrow \lambda_{t}\left(U_{p}\right)$ é folheado, isto é, $\lambda_{t}\left(L_{p} \cap U_{p}\right)=L_{\lambda_{t}(p)} \cap \lambda_{t}\left(U_{p}\right)$, ou equivalentemente, $d \lambda_{t}\left(T_{q} L_{q}\right)=T_{\lambda_{t}(q)} L_{\lambda_{t}(q)}$ para qualquer $q \in U_{p}$.

Seja $W$ um campo folheado de $\left(M, \mathcal{F}=\left\{L_{p}\right\}\right)$. Observe que $\operatorname{dim} L_{\lambda_{t}(p)}=\operatorname{dim} L_{p}$ para qualquer $p \in M$ e $t \in I_{p}$. Em particular, a curva $\lambda(t):=\lambda_{t}(p)$ está contida no conjunto $\Sigma_{k}=\left\{q \in M ; \operatorname{dim} L_{q}=k\right\}$, que chamaremos de estrato. Além disso, quando $\mathcal{F}$ é uma folheação regular os conceitos de campo folheado e campo projetável coincidem.

Definição 2.4.2 (Folheação Finsleriana singular). Seja $(M, F)$ variedade Finsler. A tripla $(M, F, \mathcal{F})$ é uma folheação Finsleriana singular quando $(M, \mathcal{F})$ for uma folheação singular $e$ $F$-transnormal, isto é, se $\gamma: I \rightarrow M$ é uma geodésica tal que $\gamma^{\prime}\left(t_{0}\right) \in \nu_{\gamma\left(t_{0}\right)} L_{\gamma\left(t_{0}\right)}$ para algum $t_{0} \in I$, então $\gamma^{\prime}(t) \in \nu_{\gamma(t)} L_{\gamma(t)}$ para qualquer $t \in I$.

Se $F$ for uma norma Riemanniana, a definição acima coincide exatamente com a definição de folheação Riemanniana singular. Porém no caso Riemanniano a folheação por esferas concêntricas de um espaço euclidiano é uma folheação Riemanniana singular, que é o modelo infinitesimal destas folheações. No caso Finsleriano, a folheação singular dada pelas indicatrizes em um espaço de Minkowski não necessariamente é Finsleriana, mais precisamente, veja o exemplo a seguir.

Exemplo 2.4.1. Sejam $M=\mathbb{R}^{2}$ e $Z$ a métrica Randers com data $(h, W)$, onde $h=\langle$,$\rangle é$ a métrica canônica e $W=\left(0, \frac{1}{2}\right)$ e $\mathcal{F}$ a folheação singular dada por $L_{p}=\mathcal{I}_{Z(p)}$, indicatriz de $(M, Z)$ com raio $Z(p)$. Considere a geodésica $\gamma(t)=(1,0)+t\left(-1, \frac{1}{2}\right)$ que é ortogonal a $L_{(1,0)}$. Porém $\gamma^{\prime}(1)=\left(-1, \frac{1}{2}\right)$ não é ortogonal a $L_{\left(0, \frac{1}{2}\right)}$, pois $\nu\left(L_{\left(0, \frac{1}{2}\right)}\right)$ é o subespaço vetorial gerado por $(0,1)$. E portanto $(M, Z, \mathcal{F})$ não é uma folheação Finsleriana singular.

Outra diferença é que enquanto no caso Riemanniano os espaços ortogonais às folhas são subespaços vetoriais, formando assim uma distribuição singular em $T M$, no caso Finsler os 
espaços ortogonais possuem apenas uma estrutura de cone. Estas diferenças motivaram a investigação do modelo local das folheações Finslerianas singulares e quais propriedades das folheações Riemannianas singulares são preservadas no caso Finsleriano.

Lema 2.4.1. Seja $(M, F, \mathcal{F})$ folheação Finsleriana singular com folhas fechadas. Se $\gamma$ : $[0,1] \rightarrow M$ é uma geodésica tal que $L(\gamma)=d\left(\gamma(0), L_{\gamma(1)}\right)$, então

$$
\left.\operatorname{dim}\left(L_{\gamma(t)}\right)=\max \left\{\operatorname{dim}\left(L_{\gamma(0)}\right), \operatorname{dim}\left(L_{\gamma(1)}\right)\right)\right\}
$$

para todo $t \in(0,1]$.

Demonstração. Se perda de generalidade, pode-se assumir que $\operatorname{dim}\left(L_{\gamma(0)}\right) \leq \operatorname{dim}\left(L_{\gamma(1)}\right)$. Suponha por absurdo que $\operatorname{dim}\left(L_{\gamma\left(t_{0}\right)}\right)<\operatorname{dim}\left(L_{\gamma(1)}\right)$ para algum $t_{0} \in(0,1)$. Logo existe geodésica $\gamma_{0}$ distinta de $\left.\gamma\right|_{\left(t_{0}, 1\right)}$ ligando $\gamma\left(t_{0}\right)$ a $L_{\gamma(1)}$ tal que $d\left(\gamma\left(t_{0}\right), L_{\gamma(1)}\right)=L\left(\gamma_{0}\right)$. Isso implica que a geodésica quebrada formada pela concatenação de $\left.\gamma\right|_{\left(0, t_{0}\right)} \operatorname{com} \gamma_{0}$ realiza a distância de $\gamma(0)$ a $L_{\gamma(1)}$, o que constitui um absurdo.

Corolário 2.4.1. Dada uma folheação Finsleriana singular com folhas fechadas, o estrato regular (isto é, conjunto dos pontos regulares) é aberto e denso.

Demonstração. Dado $p$ ponto regular, considere uma família de campos folheados $\left\{X_{1}, \ldots, X_{k}\right\}$ que é uma base de $L_{p}$ quando restrito a $T_{p} M$. Por continuidade, existe um aberto de $p$ tal que $\left\{\left.X_{1}\right|_{U}, \ldots,\left.X_{k}\right|_{U}\right\}$ continua sendo linearmente independente. Isso termina pois $p$ é ponto regular.

Dado $x \in M$ ponto singular, considere $L$ uma folha regular qualquer. Como as folhas são fechadas, existe $\gamma:[0,1] \rightarrow M$ geodésica minimizante ligando $x$ a $L$. Pelo lema anterior, $\left.\gamma\right|_{(0,1]}$ está contido dentro do estrato regular.

As próximas duas subseções apresentarão exemplos básicos de folheações Finslerianas singulares.

\section{Submersão Finsleriana}

As fibras de uma submersão Finsleriana $\pi:(M, F) \rightarrow(B, Z)$ constituem uma folheação Finsleriana singular. De fato, seja geodésica $\gamma:[0, \infty) \rightarrow M$ tal que $\gamma^{\prime}(0)$ é ortogonal a $\pi^{-1}(x)$, onde $x=\pi(p)$. Considere $V$ campo horizontal projetável sem singularidades que estende localmente $\gamma^{\prime}$ em uma vizinhança $U \subset M$ de $\gamma(0)$. Pelo Lema 1.14.3, $\pi:\left(U, g_{V}\right) \rightarrow$ $\left(\pi(U), h_{d \pi V}\right)$ é uma submersão Riemanniana, onde $g$ e $h$ são os tensores fundamentais de $F$ e $Z$ respectivamente. Observe que $\gamma$ também é uma geodésica de $\left(U, g_{V}\right) g_{V^{-}}$ortogonal a $\pi^{-1}(x)$. Logo $\gamma$ é uma geodésica $g_{V}$-horizontal, pois as fibras de $\pi$ constituem uma folheação Riemanniana com respeito a $g_{V}$. Portanto $g_{\gamma^{\prime}}\left(\gamma^{\prime}, v\right)=0$, para qualquer $v \in T_{\gamma} L$.

\section{Folheação Homogênea}

Uma ação à esquerda de um grupo de Lie $G$ sobre uma variedade $M$ é uma aplicação

$$
\begin{aligned}
\mu: G \times M & \rightarrow M \\
(g, p) & \mapsto \mu_{g}(p),
\end{aligned}
$$

tal que $\mu_{g h}(p)=\mu_{g}\left(\mu_{h}(p)\right)$ para quaisquer $g, h \in G$ e se $e \in G$ é a identidade, $\mu_{e}(p)=p$. A órbita de $\mu$ passando por $p \in M$ é o conjunto

$$
G(p)=\left\{\mu_{g}(p) \in M ; g \in G\right\},
$$


que é uma subvariedade imersa de $M$. Verifica-se que a partição de $M$ pelas órbitas de $\mu$ constitui uma folheação singular que denotaremos por $\left(M, \mathcal{F}^{G}\right)$, ou simplesmente por $\mathcal{F}^{G}$.

Agora considere uma métrica Finsleriana $F$ em $M$. Dizemos que $\mu$ é uma ação isométrica Finsleriana, se

$$
F\left(d \mu_{g} u\right)=F(u)
$$

para qualquer vetor $u \in T M$.

Exemplo 2.4.2. Seja $(V, Z)$ espaço Randers-Minkowski com data $(W, h)$. O grupo de isometrias de $(V, Z)$ é composto pelas translações $\left\{T_{v}: w \mapsto v+w ; v \in V\right\}$ e pelas rotações que deixam fixo o campo $W$. Em particular, se $W$ é não nulo, então, ao contrário do caso Riemanniano, a ação do subgrupo de isotropia da origem sobre uma indicatriz não é transitiva.

Um campo de Killing é um campo de vetores $X$ tal que seu fluxo $\varphi_{t}^{X}$ é uma isometria local, ou equivalentemente,

$$
g_{v}\left(\nabla_{u}^{v} X, w\right)+g_{v}\left(u, \nabla_{w}^{v} X\right)-2 C_{v}\left(\nabla_{v}^{v} X, u, w\right)=0,
$$

para quaisquer $u, w, v \in T_{p} M \operatorname{com} v$ não nulo, [16].

Proposição 2.4.1. Seja $\mu: G \times(M, F) \rightarrow(M, F)$ uma ação isométrica Finsleriana. Então $\mathcal{F}_{G}=\{G . p:=\mu(G, p) ; p \in P\}$, a folheação dada pelas órbitas de $\mu$, é uma folheação Finsleriana singular.

Demonstração. Dado $v \in T_{p} G$.p existe um campo de Killing $X$ da ação tal que $X(p)=v$. Observando que tais campos são tangentes as órbitas, temos que $\mathcal{F}_{G}$ é folheação singular.

Agora considere uma geodésica $\gamma:[0, a] \rightarrow M$ que é ortogonal a uma órbita G.p. É possível verificar que para qualquer $v \in T_{\gamma\left(t_{0}\right)} G \cdot \gamma\left(t_{0}\right)$ existe um campo de Killing $X$ definido em um aberto contendo $p$ e $\gamma\left(t_{0}\right)$ tal que $X\left(\gamma\left(t_{0}\right)\right)=v$. Defina a aplicação

$$
f(t)=g_{\gamma^{\prime}(t)}\left(\gamma^{\prime}(t), X\left(\gamma^{\prime}(t)\right)\right) .
$$

Provar que $\gamma$ é ortogonal as folhas é equivalente $f=0$. Pela quase compatibilidade com a métrica, por $\gamma$ ser uma geodésica e pela propriedade do tensor de Cartan (Lema 1.10.1), segue que

$$
\begin{aligned}
f^{\prime}(t) & =g_{\gamma^{\prime}(t)}\left(\gamma^{\prime}(t), \nabla_{\gamma^{\prime}(t)}^{\gamma^{\prime}} X(\gamma(t))\right)+g_{\gamma^{\prime}(t)}\left(\nabla_{\gamma^{\prime}(t)}^{\gamma^{\prime}} \gamma^{\prime}, X(\gamma(t))\right)-2 C_{\gamma^{\prime}}\left(\nabla_{\gamma^{\prime}}^{\gamma^{\prime}} \gamma^{\prime}, \gamma^{\prime}, X\right) \\
& =g_{\gamma^{\prime}(t)}\left(\gamma^{\prime}(t), \nabla_{\gamma^{\prime}(t)}^{\gamma^{\prime}} X(\gamma(t))\right)-2 C_{\gamma^{\prime}}\left(\nabla_{\gamma^{\prime}}^{\gamma^{\prime}}, \gamma^{\prime}, X\right) \\
& =g_{\gamma^{\prime}(t)}\left(\gamma^{\prime}(t), \nabla_{\gamma^{\prime}(t)}^{\gamma^{\prime}} X(\gamma(t))\right) .
\end{aligned}
$$

Por outro lado, pela equação 2.2, $2 g_{\gamma^{\prime}(t)}\left(\gamma^{\prime}(t), \nabla_{\gamma^{\prime}(t)}^{\gamma^{\prime}} X(\gamma(t))\right)=0$. Portanto $f^{\prime}(t)=0$, implicando que $f=0$, pois por suposição $f(0)=0$.

Definição 2.4.3. Uma folheação dada pelas órbitas de uma ação isométrica Finsleriana é chamada de folheação homogênea Finsleriana ou apenas folheação homogênea quando não houver risco de confusão.

\subsection{Propriedades das Folheações Finslerianas Singulares}

Nesta seção serão desenvolvidas as propriedades das folheações Finslerianas singulares necessárias para o presente trabalho, a saber: Estrutura Tubular, Lema de Homotetia, o Teorema do Slice e propriedades de Estratificação. Tais propriedades são análogas ao caso Riemannaiano, assim como algumas demonstrações. 


\subsubsection{Estrutura Tubular}

Folheações Finslerianas singulares serão caracterizadas pela a existência de uma estrutura tubular, ou seja, para uma placa fixada todas as placas suficientemente próximas estão contidas em cilindros futuros e em cilindros passados em torno desta placa fixada. Consequentemente uma folheação Finsleriana pode ser caracterizada como uma folheação cujas placas são localmente equidistantes.

Seja $P$ uma subvariedade mergulhada de $(M, F)$ variedade Finsler. O cilindro futuro e o cilindro passado de raio $r>0$ com eixo $P$ são definidos respectivamente por

$$
C_{r}^{+}(P):=\exp ^{\nu}\left(\nu^{r}(P)\right) \text { e } C_{r}^{-}(P):=\left\{\gamma_{v}(-1) \in M ; v \in \nu^{r}(P)\right\} .
$$

Quando não houver risco de confusão denotaremos apenas $C_{r}^{+}$e $C_{r}^{-}$.

Observe que a exponencial normal a uma subvariedade $P$ pode perder a suavidade em pontos distantes de $P$. Isso implica que os cilindros geométricos podem não ser uma subvariedade. Além disso, não necessariamente possuem codimensão 1.

Suponha que $P$ admite vizinhanças tubulares, digamos $T u b_{\epsilon}^{+}(P)$ e $T u b_{\epsilon}^{-}(P)$, por exemplo um aberto pré-compacto de uma subvariedade mergulhada ou subvariedade compacta. Neste caso, para $r<\epsilon$ os cilindros geométricos são hipersuperfícies definidas por

$$
C_{r}^{+}(P):=\tilde{\rho}_{+}^{-1}(r) \text { e } C^{-}(P)=\tilde{\rho}^{-1}(r),
$$

onde $\tilde{\rho}_{+}$e $\tilde{\rho}_{-}$são as restrições de $\rho_{+}:=d(P,$.$) e \rho_{-}:=d(\cdot, P)$ a $T u b_{\epsilon}^{-}(P)$ e $T u b_{\epsilon}^{+}(P)$, respectivamente. Neste texto, serão considerados apenas cilindros geométricos contidos em vizinhanças tubulares.

Lema 2.5.1. Sejam $\operatorname{Tub}_{\epsilon}^{+}(P)$ e Tub $\bar{\epsilon}^{\prime}(P)$ vizinhanças tubulares futura e passada de uma subvariedade mergulhada $P$. Se $v \in \nu^{1}(P)$, então $\gamma_{v}^{\prime}(t)$ é ortogonal a $C_{t}^{+}(P)$ e $\gamma_{v}^{\prime}(-s)$ é ortogonal a $C_{t}^{-}(P)$ para qualquer $t \in(0, \epsilon)$ e $s \in\left(0, \epsilon^{\prime}\right)$.

Demonstração. Basta provar para o caso "futuro", pois o outro caso é completamente análogo. Para qualquer $t \in(0, \epsilon)$ a geodésica $\gamma_{v}$ realiza a distância de $P$ a $\gamma_{v}(t)$. Porém $d\left(P, \gamma_{v}(t)\right)=t=d\left(P, C_{t}^{+}(P)\right)$ e portanto $\gamma_{v}^{\prime}(t)$ é ortogonal a $C_{t}^{+}(P)$, conforme Corolário $4.5 \mathrm{em}[15] \mathrm{c}$.

Observação 2.6. Como os cilindros geométricos são hipersuperfícies, o Lema anterior implica que

$$
T_{x} C_{r}^{+}(P)=\left[\mathcal{L}\left(\left(\gamma^{x}\right)^{\prime}(r)\right)\right]^{-1}(0):=\left\{w \in T_{x} M ; g_{\left(\gamma^{x}\right)^{\prime}(r)}\left(\left(\gamma^{x}\right)^{\prime}(r), w\right)=0\right\},
$$

para qualquer $x \in T u b_{\epsilon}^{+}(P)$, onde $\gamma^{x}$ é a geodésica minimizante ligando $P$ a $x$ e $r=d(P, x)$. Vale o resultado análogo para cilindro passado.

Proposição 2.6.1 (Estrutura Tubular). Seja $\mathcal{F}$ folheação singular sobre uma variedade Finsleriana $(M, F)$. Então $\mathcal{F}$ é uma folheação Finsleriana singular se, e somente se, possui uma estrutura tubular, ou seja, para cada $q \in M$ existem vizinhanças tubulares $T u b_{r}^{+}\left(P_{q}\right)$ futuro e $\operatorname{Tub}_{s}^{-}\left(P_{q}\right)$ passada de uma placa $P_{q}$ de $q$ tais que

$$
P_{x} \subset C_{\epsilon}^{+}\left(P_{q}\right) \quad \text { e } P_{x^{\prime}} \subset C_{\epsilon^{\prime}}^{-}\left(P_{q}\right),
$$

para qualquer $x \in T u b_{r}^{+}\left(P_{q}\right)$ e $x^{\prime} \in T u b_{s}^{-}\left(P_{q}\right)$, onde $\epsilon=\rho_{+}(x), \epsilon^{\prime}=\rho_{-}\left(x^{\prime}\right)$ e $P_{x} \subset L_{x} \cap$ $T u b_{r}^{+}\left(P_{q}\right)$ e $P_{x^{\prime}} \subset L_{x^{\prime}} \cap T u b_{s}^{-}\left(P_{q}\right)$ são placas passando por $x$ e $x^{\prime}$ respectivamente. 
Demonstração. Pela observação 2.6, $T_{x} P_{x} \subset T_{x} C_{\epsilon}^{+}\left(P_{q}\right)$ se, e somente se, $\gamma^{x}(\epsilon)$ é ortogonal a $T_{x} P_{x}$. E analogamente, $T_{x^{\prime}} P_{x^{\prime}} \subset T_{x^{\prime}} C_{\epsilon^{\prime}}^{-}\left(P_{q}\right)$ se, somente se, $\left(\gamma_{x^{\prime}}\right)^{\prime}\left(\epsilon^{\prime}\right)$ é ortogonal a $P_{x^{\prime}}$. Por outro lado, $P_{x} \subset C_{\epsilon}^{+}\left(P_{q}\right)$ se, e somente se, $T_{y} P_{x} \subset T_{y} C_{\epsilon}^{+}\left(P_{q}\right)$, para qualquer $y \in P_{x}$, vide Lema 2.2.1.

Agora, suponha que $\mathcal{F}$ possui uma estrutura tubular. Se $\gamma:(-b, b) \rightarrow M$ é uma geodésica unitária tal que $\gamma^{\prime}(0) \in \nu\left(L_{\gamma(0)}\right)$, considere $T u b_{r}^{+}(P)$ uma vizinhança tubular futura de $P$ placa passando por $q=\gamma(0)$. Em particular $T_{\gamma(t)} P_{\gamma(t)} \subset T_{\gamma(t)} C_{t}^{+}$para qualquer $t \in(0, r)$. Logo $\gamma^{\prime}(t)$ é ortogonal à $P_{\gamma(t)}$, implicando que $\left.\gamma\right|_{[0, b)}$ é ortogonal, pois a mesma pode ser coberta por vizinhanças tubulares futuras. Considerando vizinhanças tubulares passadas que cobrem $\left.\gamma\right|_{(-b, 0]}$, concluímos analogamente que $\left.\gamma\right|_{(-b, 0]}$ é geodésica ortogonal às folhas. E portanto $\gamma$ é geodésica ortogonal às folhas. Pela generalidade da geodésica escolhida, $\mathcal{F}$ é folheação Finsleriana singular.

Reciprocamente, suponha que $\mathcal{F}$ é Finsleriana singular. Dada uma placa $P$, para qualquer $x$ em uma vizinhança tubular futura $T u b^{+}(P)$ a geodésica ortogonal a $P$ que alcança $x$ é ortogonal a $P_{x}$. Logo $P_{x} \subset C_{d(P, x)}^{+}$para qualquer $x \in T u b^{+}(P)$. Obtemos o resultado análogo para vizinhança tubular passada. Portanto $\mathcal{F}$ possui uma estrutura tubular.

Lema 2.6.1. Se $\gamma:[0,1] \rightarrow M$ é um raio de uma vizinhança tubular $U^{+}$partindo do eixo $P_{1}$, então para qualquer $\lambda \in(0,1)$ a curva $\left.\gamma\right|_{[\lambda, 1]}$ é a única geodésica ligando $P_{\gamma(\lambda)}$ a $x=\gamma(1)$ satisfazendo a equação

$$
d\left(P_{\gamma(\lambda)}, P_{x}\right)=L\left(\left.\gamma\right|_{(\lambda, 1)}\right) .
$$

Demonstração. Dada $\gamma_{\lambda}$ geodésica minimizante ligando $P_{\gamma(\lambda)}$ a $x$ satisfazendo $L\left(\gamma_{\lambda}\right)=$ $d\left(P_{\gamma(\lambda)}, P_{x}\right)=L\left(\left.\gamma\right|_{(\lambda, 1)}\right)$, seja $\gamma_{1}:[0,1] \rightarrow M$ a geodésica radial tal que $\gamma_{1}\left(t_{0}\right)=\gamma_{\lambda}(0)$, para algum $t_{0}$. Pela estrutura tubular, $\gamma_{1}$ realiza a distância de $P_{1}$ a $P_{\gamma(\lambda)}$, implicando que $L\left(\gamma_{1}\right)=L\left(\left.\gamma\right|_{(0, \lambda)}\right)$. Considere $\gamma_{2}$ a concatenação de $\gamma_{1}$ com $\gamma_{\lambda}$. Os comprimentos de $\gamma_{2}$ e $\gamma$ coincidem:

$$
\begin{aligned}
L\left(\gamma_{2}\right) & =L\left(\gamma_{1}\right)+L\left(\gamma_{\lambda}\right)=d\left(P_{1}, P_{\gamma(\lambda)}\right)+L\left(\left.\gamma\right|_{(\lambda, 1)}\right) \\
& =L\left(\left.\gamma\right|_{(0, \lambda)}\right)+L\left(\left.\gamma\right|_{(\lambda, 1)}\right)=L(\gamma) .
\end{aligned}
$$

Assim $\gamma$ e $\gamma_{2}$ são curvas que partem ortogonalmente de $P_{1}$ e alcançam $x$, realizando a distância. Consequentemente $\gamma_{2}=\gamma$, pela definição de vizinhança tubular futura, e em particular $\gamma_{\lambda}=\left.\gamma\right|_{[\lambda, 1]}$.

Dado $\lambda \in(0,1)$ considere a aplicação $\eta: P_{\gamma(\lambda)} \rightarrow U_{r}$ dada por $\eta_{\xi}(p)=\gamma_{p}(1)$, onde $\gamma_{p}$ é a única geodésica radial de $U_{r}$ tal que $\gamma_{p}(\lambda)=p$. Observe que $\eta$ é um difeomorfismo em $\operatorname{Im}(\eta)$, pois $\operatorname{Im}(\eta)$ está contido em $U^{+}$. Pelo Lema anterior e pela estrutura tubular existe $s>0$ tal que para todo $\lambda \in(1-s, 1)$ existe $U_{\lambda}$ vizinhança aberta de $\gamma(1)$ em $P \operatorname{com} U_{\lambda} \subset \operatorname{Im}(\eta)$. Em particular, a dimensão de $P_{\gamma(\lambda)}$ coincide com a dimensão de $P_{\gamma(1)}$ e $U_{\lambda}$ está contido em uma vizinhança tubular futura de $P_{\gamma(\lambda)}$.

Lema 2.6.2. Sejam $P_{1}$ uma placa e $U$ vizinhança tubular futura de $P_{1}$ de raio $r$. Para qualquer $x \in U, \pi:=\left.\Pi\right|_{P_{x}}: P_{x} \rightarrow P_{1}$ é uma submersão. Se as placas são fechadas em $U$, então $\pi$ é sobrejetora.

Um resultado análogo vale para a aplicação que associa a cada $x$ em $U^{-}$uma vizinhança tubular passada de $P_{1}$ o ponto $\gamma_{x}(1) \in P_{1}$, onde $\gamma_{x}$ é a única geodésica em $U^{-}$tal que $\gamma_{x}(0)=x$ e $\gamma_{x}^{\prime}(1) \in \nu\left(P_{1}\right)$.

Demonstração. Pelo Lema de homotetia e pela observação 2.3, $\pi$ é uma submersão.

Em particular $\pi$ é uma aplicação aberta, e assim $\pi\left(P_{y}\right)$ é um aberto de $P_{1}$. No que se segue será demonstrado que tal conjunto também é fechado em $P_{1}$, implicando que $\pi$ é sobrejetora 
por conexidade. Dado $p$ um ponto em $P_{1}$ limite de uma sequência em $\pi\left(P_{y}\right)$, digamos $\left\{p_{n}\right\}$, seja $\left\{\gamma_{n}\right\}$ sequência de geodésicas tais que $\pi\left(\gamma_{n}(1)\right)=p_{n}$ (note que $\gamma_{n}$ é ortogonal a $L$ ). Como $P_{y}$ é pré-compacto, $\gamma_{n}(1)$ converge (passando a uma subsequência se necessário) para um ponto $y_{0}$ contido no fecho de $P_{y}$ relativo a $L_{y}$. Por continuidade $\gamma_{n}$ converge para uma geodésica $\gamma$ ortogonal a $L \operatorname{com} \gamma(0)=p, \gamma(1)=y_{0} \in P_{x}$ e $L(\gamma)=d\left(P_{1}, y_{0}\right)<r$. Como $p \in P_{1}$, segue que $\gamma \subset U_{r}$ e portanto $\pi\left(y_{0}\right)=p$.

O lema anterior implica que para quaisquer placa $P \subset U_{r}^{+}$e $p \in P_{1}$ existe uma geodésica $\gamma$ tal que $\gamma^{\prime}(0) \in \nu_{p}\left(P_{1}\right), \gamma(1) \in P$ e $L(\gamma)=d\left(P_{1}, P\right)$. E em particular $d(p, P)=d(q, P)$, para quaisquer $p, q \in P_{1}$. Por outro lado, a estrutura tubular, implica que $d\left(P_{1}, x\right)=d\left(P_{1}, y\right)$ para quaisquer $x, y \in P$. Resultados análogos valem para $d\left(P, P_{1}\right)$, considerando apenas vizinhança tubular passada. Porém quando as folhas são conexas e fechadas estes resultados são verdadeiros não apenas para pontos em uma vizinhança de uma placa, como afirma o lema a seguir.

Lema 2.6.3. Seja $(M, F, \mathcal{F})$ uma folheação Finsleriana singular com folhas conexas e fechadas. Dada uma folha $L$ segue que $d(x, L)=d(y, L)$ e $d(L, x)=d(L, y)$, para quaisquer $x, y \in L_{0}$.

Demonstração. Será demonstrado apenas para a primeira equação, pois a demonstração para a segunda é análoga.

Primeiro suponha que $L$ e $L_{0}$ são folhas regulares. Como as folhas de $\mathcal{F}$ são fechadas, existe $\gamma:[0,1] \rightarrow M$ geodésica tal que $\gamma(0)=x, \gamma(1) \in L$ e $L(\gamma)=d\left(x, L_{0}\right)$.

A geodésica $\gamma$ não contém pontos singulares em seu interior. Suponha por absurdo que para algum $\epsilon \in(0,1)$ o ponto $p=\gamma(\epsilon)$ é singular. Logo pela estrutura tubular e por $\operatorname{dim}\left(L_{p}\right)<d(L)$, existe uma geodésica $\beta$ distinta de $\left.\gamma\right|_{(\epsilon, 1)}$ ligando $p$ a $L$ realizando a distância. Isso implica que a concatenação de $\left.\gamma\right|_{(0, \epsilon)} \operatorname{com} \beta$ é uma geodésica quebrada que realiza a distância de $x_{0}$ a $L$, o que constitui um absurdo.

A afirmação no início do parágrafo anterior mais a conexidade das folhas implicam que $\gamma$ pode ser transportada para uma geodésica $\gamma_{0}$ tal que $\gamma_{0}(0)=y, \gamma_{0}(1) \in L$ e $L\left(\gamma_{0}\right)=L(\gamma)$, terminando a prova deste caso. Para verificar isso basta que o fato da folheação no estrato regular ser descrita localmente por submersões Finslerianas permite considerar apenas o caso em que $L$ e $L_{0}$ são fibras de uma submersão de uma mesma Finsleriana. E neste caso, basta considerar $\gamma_{0}$ como o levantamento horizontal de $\gamma$ partindo de $y$.

Por fim se $L$ e $L_{0}$ são folhas singulares, basta usar o caso anterior e que fato de que o estrato regular é um aberto denso.

\subsubsection{Lema de Homotetia}

Aqui será provado que localmente qualquer folheação Finsleriana singular é invariante por homotetia e seguirá como corolário que uma folheação regular é Finsleriana se, e somente se, é descrita localmente por submersões Finslerianas.

Seja $P$ uma subvariedade mergulhada pré-compacta de $(M, F)$. Dado $\lambda>0$, seja $r>0$ tal que existam vizinhanças tubulares futuras com raio $r$ e $\lambda r$. De fato sempre existe o número $r$, basta tomar qualquer vizinhança tubular futura $T u b_{s}^{+}(P)$ e considerar $r=s$, se $\lambda \leq 1$ ou $r=\frac{1}{s}$, se $\lambda>1$. O resultado análogo vale para vizinhança tubular passada. Neste caso, defini-se a aplicação homotetia futura por

$$
\begin{aligned}
h_{\lambda}^{+}: T u b_{r}^{+}(P) & \rightarrow T u b_{\lambda r}^{+}(P) \\
x & \mapsto h_{\lambda}^{+}(x)=\gamma_{x}(\lambda),
\end{aligned}
$$


onde $\gamma_{x}$ é a geodésica ortogonal a $P$ em $\gamma_{x}(0)$ tal que $\gamma_{x}(1)=x$. Analogamente definimos a homotetia passada como

$$
\begin{aligned}
h_{\lambda}^{-}: T u b_{r}^{-}(P) & \rightarrow T u b_{\lambda r}^{-}(P) \\
x & \mapsto h_{\lambda}^{-}(x)=\gamma^{x}(-\lambda),
\end{aligned}
$$

onde $\gamma^{x}$ é a geodésica minimizante tal que $\gamma^{x}(0)=x$ e $\left(\gamma^{x}\right)^{\prime}(1) \in \nu\left(P_{q}\right)$. Em particular, $h_{\lambda}^{ \pm}\left(C_{\epsilon}^{ \pm}\left(P_{q}\right)\right)=C_{\lambda \epsilon}^{ \pm}\left(P_{q}\right)$ para qualquer $\epsilon \in(0, r)$. Observe também que

$$
h_{\lambda \beta}^{ \pm}=h_{\lambda}^{ \pm} \circ h_{\beta}^{ \pm},
$$

para quaisquer números positivos $\lambda$ e $\beta$ que façam sentido a definição de homotetia passada ou futura. Em particular $h_{\lambda}^{ \pm}: T u b_{r}^{ \pm}(P) \rightarrow T u b_{\lambda r}^{ \pm}(P)$ é um difeomorfismo folheado para a folheação por cilindros futuros/passados, cuja inversa é dada por

$$
\left(h_{\lambda}^{ \pm}\right)^{-1}=h_{\frac{1}{\lambda}}^{ \pm}: T u b_{\lambda r}^{ \pm} \rightarrow T u b_{r}^{ \pm} .
$$

Proposição 2.6.2 (Lema de Homotetia). A aplicação de homotetia futura $h_{\lambda}^{+}: T u b_{r}^{+}\left(P_{q}\right) \rightarrow$ $T u b_{\lambda r}^{+}\left(P_{q}\right)$ (respectivamente passada) é um difeomorfismo folheado, ou seja, $h_{\lambda}^{+}\left(P_{x}\right)=P_{h_{\lambda}(x)}$ para qualquer placa $P_{x}$ em $T u b_{r}^{+}\left(P_{x}\right)$.

Demonstração. Basta provar o resultado para a homotetia futura $h_{\lambda}:=h_{\lambda}^{+}$, pois a argumentação para a homotetia passada é análoga. Denote por $U_{r}=T u b_{r}^{+}\left(P_{q}\right)$ a vizinhança tubular futura de $P_{q}$ com raio $r$. Observe que $h_{\lambda}$ está bem definido em $U_{r}$ para qualquer $\lambda \leq 1$.

Pelo Lema 2.6.1 existe $s>0$ tal que $x$ está contido em alguma vizinhança tubular de $P_{h_{\lambda}(x)}$, denotada por $V_{\lambda}$ para todo $\lambda \in(1-s, 1)$.

Afirmação 1. Dado $x \in U_{r}$ existe $U_{x}$ vizinhança aberta de $x$ em $P_{x}$ tal que $h_{\lambda}\left(U_{x}\right) \subset P_{h_{\lambda}(x)}$, para qualquer $\lambda \in(1-s, 1)$.

Neste parágrafo será demonstrada a afirmação acima. Dado $\lambda \in(1-s, 1)$, tome o aberto $U_{x}=P_{x} \cap V_{\lambda}$. Dado $y \in U_{x}$, existe $\gamma_{1}:\left[0, r_{2}\right] \rightarrow V_{\lambda}$ geodésica unitária minimizante tal que $\gamma_{1}(0) \in P_{h_{\lambda}(x)}$ e $\gamma_{1}\left(r_{2}\right)=y$, onde $r_{2}:=d\left(P_{h_{\lambda r_{1}}(x)}, P_{x}\right)=(1-\lambda) r_{1}$ e $r_{1}:=d\left(P_{q}, P_{x}\right)$. Agora considere $\gamma_{2}:\left[0, r_{1}\right] \rightarrow U_{r}$ geodésica unitária minimizante tal que $\gamma_{2}(0) \in P_{q}$ e $\gamma_{2}\left(\lambda r_{1}\right)=\gamma_{1}(0)$. Seja $\gamma:\left[0, r_{1}\right] \rightarrow U_{r}$ a concatenação de $\gamma_{2}$ com $\gamma_{1}$ que a princípio é apenas uma geodésica quebrada. Porém como $\gamma$ conecta $P_{q}$ a $P_{x}$ realizando a distância $d\left(P_{q}, P_{x}\right)$, segue que $\gamma$ de fato é uma geodésica unitária minimizante que parte ortogonalmente de $P_{q}$, $\operatorname{logo} h_{\lambda}(y)=\gamma\left(\lambda r_{1}\right) \in P_{h_{\lambda(x)}}$.

A afirmação anterior implica trivialmente que o conjunto $\mathcal{A}=\left\{y \in P_{x} ; h_{\lambda}(y) \in P_{h_{\lambda}(x)}\right\}$ é um aberto de $P_{x}$. Ela também implica que é fechado, pois se $y \notin \mathcal{A}$ existe $U$ vizinhança aberta de $y$ em $P_{x}$ tal que $h_{\lambda}(U) \cap P_{h_{\lambda}(x)}=\emptyset$. Logo pela conexidade das placas, $\mathcal{A}=P_{x}$, e portanto,$h_{\lambda}\left(P_{x}\right) \subset P_{h_{\lambda}(x)}$, para qualquer $\lambda \in(1-s, 1)$.

Analogamente garante-se a existência de $s_{0}>0$ tal que $h_{\lambda}\left(P_{x}\right) \subset P_{h_{\lambda}(x)}$ para qualquer $\lambda \in\left[1,1+s_{0}\right)$. Supondo sem perda de generalidade que $s<s_{0}$, segue que esta inclusão é satisfeita para qualquer $\lambda \in I_{x}:=\left(1-s, 1+\frac{1}{s}\right)$. Um argumento análogo também garante a existência de um intervalo $J_{x}$ contendo 1 tal que $P_{h_{\lambda}(s)} \subset h_{\lambda}\left(P_{x}\right)$ para qualquer $\lambda \in J_{x}$. E portanto $P_{h_{\lambda}(s)}=h_{\lambda}\left(P_{x}\right)$ para todo $\lambda \in N_{x}:=I_{x} \cap J_{x}$.

Considere o conjunto $\mathcal{B}=\left\{\lambda ; h_{\lambda}\left(P_{x}\right)=P_{h_{\lambda}(x)}\right\}$, que é fechado por continuidade. Para concluir a prova, resta verificar que $\mathcal{B}$ é aberto. Dado $\lambda \in \mathcal{B}$, considere $N_{h_{\lambda}(x)}$ que é um intervalo aberto contendo 1 . Logo $\lambda . N_{h_{\lambda}(x)}$ é um intervalo aberto contendo $\lambda$. Se $\eta=\lambda . \rho$ $\operatorname{com} \rho \in N_{h_{\lambda}(x)}$, então por definição $h_{\lambda \rho}\left(P_{x}\right)=h_{\rho}\left(P_{h_{\lambda}(x)}\right)=P_{h_{\lambda \rho}(x)}$, e portanto $\rho \in \mathcal{B}$. 
Observação 2.7. Observe que as aplicações homotetia aplicam difeomorficamente placa em placa, pois dois raios distintos partindo de uma placa não se encontram dentro de uma vizinhança tubular.

Corolário 2.7.1. Seja $(M, F, \mathcal{F})$ uma folheação Finsleriana singular com folhas conexas dadas pelas fibras de uma submersão $\pi: M \rightarrow N$. Então existe uma única métrica Finsleriana $Z$ em $N$ tal que $\pi:(M, F) \rightarrow(N, Z)$ é uma submersão Finsleriana.

Demonstração. Defina a seguinte aplicação $Z: T N \rightarrow \mathbb{R}$ por $Z:=F \circ \varphi^{-1}$, onde $\varphi^{-1}$ é o levantamento horizontal de $\pi$ (Proposição 1.14.2). Observe que para cada $p \in M, Z_{p}=$ $F_{p} \circ \varphi_{p}^{-1}$ é a única métrica Finsleriana tal que $d \pi_{p}:\left(T_{p} M, F\right) \rightarrow\left(T_{\pi(p)} N, Z\right)$ é uma submersão Finsleriana. Logo para terminar a prova basta verificar que tal aplicação está bem definida, ou seja, $F$ restrito a $d \pi^{-1}(w)$ é constante para qualquer $w \in T N$. Dados $v_{1}, v_{2} \in \pi^{-1}(w)$, pela conexidade das fibras podemos considerar que $\rho\left(v_{1}\right)$ e $\rho\left(v_{2}\right)$ estão contidos em uma mesma placa, digamos $P_{0}$. Sejam $x=\gamma_{v_{1}}(s)$ contido em uma vizinhança tubular futura de $P_{0}$ e $P_{1}$ placa contendo $\gamma_{v_{1}}(s)$. Seja $\gamma$ geodésica minimizante partindo de $\rho\left(v_{2}\right)$ até $P_{1}$. Note que pela estrutura tubular, $F\left(v_{1}\right)=F\left(\gamma^{\prime}(0)\right)$. Além disso, pelo Lema de homotetia, $\pi \circ \gamma=\pi \circ \gamma_{v_{1}}$ e em particular $d \pi v_{1}=d \pi \gamma^{\prime}(0)$. E pela unicidade do levantamento horizontal $v_{2}=\gamma^{\prime}(0)$. Portanto $F\left(v_{2}\right)=F\left(\gamma^{\prime}(0)\right)=F\left(v_{1}\right)$.

Proposição 2.7.1. Uma folheação singular $(M, F, \mathcal{F})$ que possui apenas folhas regulares $e$ conexas é F-transnormal se, e somente se, for uma folheação Finsleriana.

Demonstração. Basta observar que qualquer folheação singular com folhas de mesma dimesão é uma folheação regular (isto é, dada localmente por submersões) e usar o Corolário anterior.

\subsubsection{Teorema do Slice e Folheação Infinitesimal}

Dada folheação singular $(M, \mathcal{F})$ um slice por $q \in M$ é uma subvariedade mergulhada $S_{q}$ de $M$ tal que $T_{q} M=T_{q} L_{q} \oplus T_{q} S_{q}$ e $T_{p} M=T_{p} L_{p}+T_{p} S_{q}$. A folheação $\mathcal{F}_{q}$ induzida em um slice $S_{q}$ será denominada de folheação slice por $q$, mais precisamente a folha de $\mathcal{F}_{q}$ passando por $p \in S_{q}$ é definida por $L_{p}^{S}:=L_{p} \cap S_{q}$.

Teorema 2.7.1 (Folheação Slice). Seja $(M, F, \mathcal{F})$ uma folheação Finsleriana singular. Então para qualquer $q \in M$ existem uma vizinhança $U$ de uma placa $P_{q}$, um slice $S=S_{q}$ de $\left(U,\left.\mathcal{F}\right|_{U}\right)$ passando por q e uma métrica Finsleriana $Z_{S}$ em $S$ tais que a folheação slice $\mathcal{F}_{q}:=\{L \cap S\}$ é uma folheação Finsleriana singular em $\left(S, Z_{S}\right)$, onde q é uma folha 0dimensional, e satisfaz a equação

$$
d_{F}\left(P_{p}, P_{z}\right)=d_{Z_{S}}\left(P_{p} \cap S, P_{z} \cap S\right),
$$

para quaisquer placas $P_{p}$ e $P_{z}$ de $(M, F, \mathcal{F})$ com $p, z \in S$.

Demonstração. Dado $q \in M$ considere $\left(U, \mathcal{F}^{2}\right)$ uma subfolheação regular de $\mathcal{F}$ e $S$ o slice passando por $q$ dados no Lema 2.2.2. Seja $\pi: U \rightarrow S$ a submersão definida por $\pi(p)=L_{p}^{2} \cap S$. Defina a métrica Finsleriana em $S$ dada por $\left(Z_{S}\right)_{p}:=F \circ \varphi_{p}^{-1}$ para qualquer $p \in S$, onde $\varphi_{p}$ é o levantamento $F$-horizontal de $\pi$. Observe que $d \pi_{p}:\left(T_{p} M, F_{p}\right) \rightarrow\left(T_{p} S,\left(Z_{S}\right)_{p}\right)$ é uma submersão Finsleriana para qualquer $p \in S$, Lema 1.14.1.

Dado $p \in S$ seja $U_{0}$ vizinhança tubular futura da placa $P_{p}$ e $U_{S}=U_{0} \cap S$. Considere a função transnormal $f(x)=d_{F}\left(P_{p}, x\right)$ e $f_{S}(x):=\left.f\right|_{S}$ sua restrição a $S$. Como $\left(U_{0}, F, \mathcal{F}\right)$ é folheação Finsleriana singular, as placas $P_{x}$ em $U_{0}$ formam uma subfolheação da folheação 
singular $\mathcal{C}=\left\{C_{r}=f^{-1}(r)\right\}$, que são os $F$-cilindros futuros com eixo $P_{p}$. Logo as placas de $\mathcal{F}_{S}$ em $U_{S}$ estão contidas nas hipersuperfícies de nível da função $f_{S}$. No que se segue será provado que $f_{S}$ é uma função $Z_{S^{-}}$transnormal cuja norma do $Z_{S}$ gradiente é igual a norma do $F$-gradiente de $f$. Por analogia obtém-se o resultado análogo para os cilindros passados, implicando assim o resultado pela Proposição 2.6.1.

Dado $X$ o gradiente de $f$, que é definido em $U_{0} \backslash P_{p}$, defina o campo suave $X_{S}(x)=\pi_{x}(X)$ em $\left(U_{0} \backslash P_{p}\right) \cap S$. Como $\pi_{x}:\left(T_{x} U_{0}, F\right) \rightarrow\left(T_{x},\left(Z_{S}\right)_{x}\right)$ é submersão Finsleriana para cada $x \in S$, tem-se que

1. $F(X(p))=Z_{S}\left(X_{S}(p)\right)$ para qualquer $p \in S$; e

2. $g_{X}^{F}(X, v)=g_{X_{S}}^{Z_{S}}\left(X_{S}, v\right), \forall v \in T S$.

De fato, a primeira igualdade segue diretamente do fato de $X$ ser $F$-ortogonal às placas da folheação $\mathcal{F}^{2}$, pois tais placas estão contidas nas hipersuperfícies de níveis de $f$. Este fato e a Proposição 1.14.3 implicam a equação (2):

$$
g_{X}^{F}(X, v)=g_{X}^{F}\left(X, \pi_{x}(v)\right)=g_{X_{S}}^{Z_{S}}\left(X_{S}, \pi_{x}(v)\right)=g_{X_{S}}^{Z_{S}}\left(X_{S}, v\right),
$$

para qualquer $v \in T_{x} S$.

Por sua vez, a equação (2) implica que $X_{S}$ é o $Z_{S^{-}}$gradiente da função $f_{S}$, pois trivialmente $d f(v)=d f_{S}(v)$ para qualquer $v \in T S$. Logo, pela equação (1), $f$ é uma função $Z_{S^{-}}$transnormal com

$$
d_{Z_{S}}\left(P_{p} \cap S, x\right)=\int_{0}^{r} \frac{1}{\sqrt{Z_{S}\left(X_{S}\right)}}=\int_{0}^{r} \frac{1}{\sqrt{F(X)}}=d\left(P_{p}, x\right),
$$

para qualquer $x \in S$, pelo Teorema 1.15.1.

Dado $\lambda<1$, considere a métrica $Z_{\lambda}^{0}=\left(h_{\lambda}^{+}\right)^{*} Z_{S}$. Neste caso, $\gamma$ é uma geodésica de $Z_{\lambda}^{0}$ se, e somente se, $\tilde{\gamma}:=h_{\lambda}^{+} \circ \gamma$ é uma geodésica de $Z_{S}$. Além disso, pelo Lema de homotetia, $\gamma(t)$ é $Z_{\lambda}^{0}$-ortogonal a $L_{\gamma(t)}$ se, e somente se, $\tilde{\gamma}(t)$ é $Z_{S^{-}}$ortogonal a $L_{\tilde{\gamma}(t)}$ para qualquer $t$. Agora considera a métrica $Z_{\lambda}:=\frac{1}{\lambda} h_{\lambda}^{*} Z_{S}$. Pela discussão acima e pela Observação 1.11, $\gamma$ é uma geodésica de $Z_{\lambda}$ se, e somente se, $\tilde{\gamma}$ é geodésica de $Z_{S}$; e $\gamma(t)$ é $Z_{\lambda}$-ortogonal a $L_{\gamma(t)}$ se, e somente se, $\tilde{\gamma}(t)$ é $Z_{S}$-ortogonal a $L_{\tilde{\gamma}(t)}$ para qualquer $t$.

Lema 2.7.1. Sejam $\left(S_{q}, Z_{S}, \mathcal{F}_{q}\right)$ uma follheação Slice de uma folheação Finsleriana singular $(M, Z, \mathcal{F})$ e $h_{\lambda}$ a restrição da aplicação homotetia futura com respeito a placa $P_{q}$ ao Slice $S_{q}$. Então $\left(S_{q}, Z_{\lambda}, \mathcal{F}_{q}\right)$ é uma folheação Finsleriana singular, onde $Z_{\lambda}:=\frac{1}{\lambda} h_{\lambda}^{*} Z_{S}$.

Demonstração. Se $\gamma$ é uma geodésica ortogonal a $L_{\gamma(0)}$ com respeito a $Z_{\lambda}$, então $\tilde{\gamma}:=h_{\lambda} \circ \gamma$ é geodésica ortogonal a $L_{\tilde{\gamma}(0)}$ com respeito a $Z_{S}$. Como a folheação Slice é Finsleriana singular, $\tilde{\gamma}(t)$ é ortogonal a $L_{\tilde{\gamma}(t)}$ com respeito a $Z_{S}$ e portanto $\gamma(t)$ é $Z_{\lambda}$-ortogonal a $L_{\gamma(t)}$.

Uma folheação Finsleriana singular é dita localmente fechada quando para qualquer $p \in$ $M$ a folheação Slice passando por $p$ possui apenas folhas compactas.

Proposição 2.7.2 (Folheação Infinitesimal). Seja $(M, F, \mathcal{F})$ uma folheação Finsleriana singular localmente fechada. Então para qualquer slice $S=S_{q}$ existe um difeomorfismo folheado $f: S \rightarrow \mathbb{R}^{k}$ entre a folheação slice $\left(S, Z_{S}, \mathcal{F}_{q}\right)$ e uma folheação Finsleriana singular em um espaço Minkowski tal que $f(q)=0$ é uma folha 0-dimensional, que chamaremos de folheação infinitesimal. 
Demonstração. Nesta demonstração a exponencial com respeito a $Z_{S}$ será denotada por exp.

No espaço Finsleriano $\left(S_{q}, Z_{S}\right)$ defina a transformação homotetia $h_{\lambda}: S_{q} \rightarrow S_{q}, h_{\lambda}(x)=$ $\gamma_{x}(\lambda)$ onde $\gamma_{x}$ é a geodésica radial em $\left(S_{q}, Z_{S}\right)$ tal que $\gamma_{x}(0)=q$ e $\gamma_{x}(1)=x$. Considerando a métrica $Z_{\lambda}:=\frac{1}{\lambda} h_{\lambda}^{*} Z_{S}$, observe que $\lim _{\lambda \rightarrow 0} Z_{\lambda}$ converge uniformemente para uma métrica Finsleriana $Z_{0}$ em $S_{q}$. Verifica-se que $\left(\exp _{q}^{-1}\right)^{*}\left(Z_{S}\right)=\left(Z_{0}\right)$. De fato, dado $v \in T_{x} M$,

$$
\begin{aligned}
\frac{1}{\lambda} h_{\lambda}^{*} Z_{S}(v) & =Z_{S}\left(\frac{1}{\lambda} d\left(h_{\lambda}\right)_{x} v\right)=Z_{S}\left(\frac{1}{\lambda} d\left(\exp _{q}\right)_{\lambda \exp _{q}^{-1}(x)} \lambda d\left(\exp _{q}^{-1}\right)_{x}(v)\right) \\
& =Z_{S}\left(d\left(\exp _{q}\right)_{\lambda \exp _{q}^{-1}(x)} d\left(\exp _{q}^{-1}\right)_{x}(v)\right),
\end{aligned}
$$

e como $d\left(\exp _{q}\right)_{\lambda \exp p_{q}^{-1}(x)} \rightarrow d\left(\exp _{q}\right)_{0}=I d$, quando $\lambda \rightarrow 0$, segue que $Z_{0}=\left(\exp _{q}^{-1}\right)^{*} Z_{S}$. Resta verificar que $\left(S, Z_{0}, \mathcal{F}_{q}\right)$ é folheação Finsleriana singular. Fixe as folhas $L_{x}$ e $L_{0}$. Pelos Lemas 2.7.1 e 2.6.3, $d_{\lambda}\left(L_{0}, x\right)=d\left(L_{0}, y\right)$ para quaisquer $y \in L_{x}$ e $\lambda$. Fazendo $\lambda$ tender a 0 , segue que $d_{0}\left(L_{0}, x\right)=d_{0}\left(L_{0}, y\right)$, onde $d_{0}$ é a aplicação distância referente a $Z_{0}$. Procedendo analogamente, segue que $d_{0}\left(x, L_{0}\right)=d_{0}\left(y, L_{0}\right)$. Isso implica do resultado.

\subsubsection{Estratificação}

Seja $\left(M^{n}, \mathcal{F}^{k}\right)$ uma folheação singular de dimensão $k$. Dado $r \in\{0,1, \ldots, k-1, k\}$ definimos o estrato $r$-dimensional por $\Sigma_{r}:=\left\{p \in M ; \operatorname{dim} L_{p}=r\right\}$, ou seja, o conjunto formado por todas as folhas de dimensão $r$. O estrato de dimensão máxima $\Sigma_{k}$ é chamado de estrato regular, enquanto o estrato de dimensão mínima é o estrato mínimo.

Lema 2.7.2. Seja $\left(\mathbb{R}^{n}, Z, \mathcal{F}\right)$ folheação Finsleriana singular em um espaço Minkowski que possui uma folha 0-dimensional. Então o estrato mínimo $\Sigma$ é um subespaço vetorial.

Demonstração. Primeiro observe que a homotetia de uma folha mínima está definida para todo instante em $[0, \infty)$. Suponha sem perda de generalidade que $0 \in \Sigma$. Logo para terminar a prova basta verificar que $\Sigma$ é fechado para a soma de vetores. Dados $v, w \in \Sigma$, o lema de homotetia implica que $s v, s w \in \Sigma$, para qualquer $s>0$. Considere a curva $\beta:[0,1] \rightarrow V$ dada por $\beta(t)=2(v+t(w-v))$. Note que $\beta$ é o segmento de reta partindo de $2 v$ passando por $v+w$ em $t=\frac{1}{2}$ e alcançando $2 w$. Considerando a homotetia em $2 v$, concluímos que $\beta \subset \Sigma$ pois $2 w \in \Sigma$, conforme a observação anterior. Logo em particular $v+w \in \Sigma$.

Proposição 2.7.3. Seja $\left(M^{n}, F, \mathcal{F}^{k}\right)$ uma folheação Finsleriana singular $k$-dimensional. Então $\Sigma_{r}$ é uma união disjunta de subvariedades mergulhadas de mesma dimensão, para qualquer $r \in\{0, \ldots, k\}$ e $\left\{\Sigma_{r}\right\}$ é uma estratificação. Além disso, a folheação induzida em qualquer estrato é uma folheação Finsleriana (regular).

Demonstração. Fixe um estrato $\Sigma_{r}$. Dada $q \in \Sigma_{r}$, seja $\left(S_{q}, F, \mathcal{F}_{q}\right)$ uma folheação slice. Observe que $q$ está contido no estrato mínimo de $\mathcal{F}_{q}$ e que este estrato mínimo é difeomorfo ao estrato mínimo da folheação infinitesimal. Logo pelo lema anterior, a componente conexa do estrato mínimo de $\mathcal{F}_{q}$ é uma subvariedade mergulhada. Por fim, observe que $\Sigma_{r}$ em torno de $q$ é identificada com o produto da placa $P_{q}$ com o estrato mínimo de $\mathcal{F}_{q}$. Isso implica que a componente conexa de $\Sigma_{r}$ é uma subvariedade mergulhada.

Dada placa $P \subset \Sigma_{r}$ seja $U$ uma interseção de uma vizinhança tubular futura com uma passada. A seguir será verificado que qualquer placa $P_{0} \subset U \cap \Sigma_{r}$ é equidistante a $P$. Dado $y \in P_{0}$ existe $\gamma^{y}$ geodésica horizontal de $M$ ligando $P$ a $y$ tal que $L\left(\gamma^{y}\right)=d\left(P, P_{0}\right)$. Pelo lema de homotetia $\gamma^{y}(t) \in \Sigma_{r}$ para qualquer $t \in(0,1)$. Logo $\gamma^{y}$ é uma geodésica ortogonal de $\Sigma_{r} \mathrm{e}$

$$
d_{\Sigma_{r}}(P, y)=L\left(\gamma^{y}\right)=d(P, y)
$$


Pela generalidade da escolha de $y \in P_{0}$ e por $\mathcal{F}$ ser folheação Finsleriana singular, concluímos que $P$ é paralelo a $P_{0}$ em $M$. Usando um argumento análogo, concluímos que $P_{0}$ é paralelo a $P$ em $M$. Logo $P$ é equidistante a $P_{0}$ e portanto $\left(\Sigma_{r},\left.F\right|_{\Sigma_{r}}, \mathcal{F}\right)$ é uma folheação Finsleriana singular, pela Proposição 2.6.1.

Prova da Proposição 2.0.2. Seja $U$ uma vizinhança tubular de uma placa $P_{q} \subset \Sigma_{r}$. Observe que o resultado é válido para o estrato regular e consequentemente, pode ser assumido que o resultado é verdadeiro para $\cup_{s>r} \Sigma_{s}$.

Seja $\tilde{\gamma}$ geodésica ortogonal a $P_{q}$ com respeito a $h$ com $\tilde{\gamma}(0)=q$. Primeiro suponha que $\tilde{v}:=\tilde{\gamma}^{\prime}(0)$ não é tangente $\Sigma_{r}$.

Dado $V_{0} \subset T_{q} M$ o hiperplano definido como o subespaço $h$-ortogonal a $\tilde{v}$, considere $\gamma$ a geodésica com respeito a $F$ tal que $v:=\gamma^{\prime}(0)$ é $F$-ortogonal a $V_{0}$ e aponta para o mesmo lado que $\tilde{v}$.

Considere o caso em que $v$ não é tangente a $\Sigma_{r}$. Para cada $t$ defina $V_{t}$ como o espaço tangente ao cilindro futuro (com respeito a $F$ ) com eixo $P_{q}$ em $\gamma(t)$. Como $(M, F, \mathcal{F})$ é folheação Finsleriana singular, $T_{\gamma(t)} L_{\gamma(t)} \subset V_{t}$. Defina $\tilde{\gamma}_{t}$ como a geodésica de $(M, h) h$ ortogonal a $V_{t}$ tal que $v_{t}=\tilde{\gamma}_{t}^{\prime}(0)$ está do mesmo lado que $\gamma^{\prime}(t)$. Então $\gamma_{t}$ é ortogonal a qualquer folha em $\cup_{s>r} \Sigma_{s} \cap U$, pois $\left(\cup_{s>r} \Sigma_{s} \cap U, h, \mathcal{F}\right)$ é folheação Riemanniana singular, por hipótese de indução. E como $\gamma_{t}$ converge para $\gamma$ segue que $\gamma$ é $h$-ortogonal às folhas em $\cup_{s>r} \Sigma_{s} \cap U$. Agora suponha que $v$ é tangente a $\Sigma_{r}$. Neste caso, considere uma sequência de geodésicas $\gamma_{i}$ em $\cup_{s>r} \Sigma_{s} \cap U$ tais que $\gamma_{i}^{\prime}(0)$ é ortogonal a $P_{q}$ e não é tangente a $\Sigma_{r}$ para qualquer $i$. Assim o resultado segue da convergência e do caso anterior.

Resta considerar quando $\tilde{v}$ é tangente $\Sigma_{r}$. Será verificado que $\tilde{\gamma} \subset \Sigma_{r}$, implicando que $\tilde{\gamma}$ é $h$-ortogonal às folhas, pois por hipótese $\left(\Sigma_{r}, h, \mathcal{F}\right)$ é folheação Finsleriana singular. Defina $B=\Sigma_{r} \cap \exp \left(\nu\left(P_{q}\right)\right) \cap U$. Para terminar a prova, basta verificar que $B$ coincide com $A:=$ $\exp \left(T_{q} \Sigma_{r} \cap \nu\left(P_{q}\right)\right) \cap U$. Seja $x \in B$, isto é, $x=\exp \left(t_{x} v\right) \in \Sigma_{r} \cap U$ para algum $t_{x}>0$ e $v \in \nu\left(P_{q}\right)$. Mas pelo Lema de homotetia as dimensões de $P_{\exp (t v)}$ e $P_{x}$ coincidem para quaisquer $t \in\left(0, t_{x}\right)$. Em particular, $v \in T_{q} \Sigma_{r}$ e portanto $x \in A$. Obviamente $B$ é aberto de $A$. Por outro lado, $B$ também é um subconjunto fechado de $A$. De fato, seja $x$ pertencente ao fecho de $B$ em $A$, ou seja, $x \in A$ e existe uma sequência de geodésicas $\gamma_{n}$ ortogonais a $P_{q}$ contidas em $\Sigma_{r} \cap U$ tais que $\gamma_{n}(1)$ convergem para $x$. Observando que $\gamma_{n}$ converge para uma geodésica de $\Sigma_{r}$ ortogonal a $P_{q}$, conclui-se que $x \in B$. Como $A$ é conexo, segue que $A=B$. 
52 FOLHEAÇÕES FINSLERIANAS SINGULARES, DEFINIÇÕES E PROPRIEDADES 


\section{Capítulo 3}

\section{Folheações Randers Singulares}

Uma folheação Randers singular com data $(h, W)$ é uma folheação Finsleriana singular sobre a variedade Randers com data $(h, W)$. Este é objeto de estudo deste capítulo, sendo o principal objetivo provar o teorema abaixo.

Teorema 3.0.2. Se $\mathcal{F}$ é uma folheação Randers singular com data $(h, W)$ sobre uma variedade $M$, então o vento $W$ é um campo folheado e $(M, h, \mathcal{F})$ é uma folheação Riemanniana singular.

A estratégia para provar este teorema será provar o resultado para o caso regular, estudar folheações Finslerianas singulares em espaço Randers-Minkowski tendo a origem como uma folha, usar este estudo e o Teorema do Slice 2.0.2 para provar que $W$ é tangente aos estratos, o que implica que a folheação restrita aos estratos é uma folheação Randers regular e por fim usar a Proposição 2.0.2.

Conjectura-se que a recíproca deste resultado é verdadeira, o que seria uma generalização do problema de navegação de Zermelo. Esta conjectura será verificada nos seguintes casos particulares: folheação Randers regular, folheação Randers homogênea e folheação Randers singular em um ambiente com curvatura flag constante.

Na primeira seção, apresentaremos casos particulares, dentre os quais provaremos a caracterização das folheações Randers regulares. Na segunda estudaremos folheações Randers singulares em espaços Randers-Minkowski, na terceira provaremos o resultado principal. E na última construiremos uma família infinita de folheações Randers singulares não-homogêneas.

\subsection{Casos Particulares}

Nesta seção serão demonstrados casos particulares do Teorema principal com sua recíproca e sua recíproca para o caso em que o vento é infinitesimalmente homotético.

O primeiro dos casos será o único utilizado na demonstração do Teorema 1. Logo caso o leitor queira pode omitir as duas últimas subseções desta seção.

Teorema 3.1.1. Sejam $\pi: M \rightarrow B$ submersão e $Z$ métrica Randers com data $(h, W)$. Então $\pi:(M, Z) \rightarrow(B, F)$ é uma submersão Finsleriana se, e somente se, for a translação de uma submersão Riemanniana. Em particular,

1. W é um campo projetável; e

2. F é uma métrica Randers. 
Demonstração. Para cada $p \in M$ seja $g_{\pi(p)}: T_{\pi(p)} N \times T_{\pi(p)} N \rightarrow \mathbb{R}$ a única métrica Riemanniana tal que $d \pi_{p}:\left(T_{p} M, h_{p}\right) \rightarrow\left(T_{\pi(p)} N, g_{\pi(p)}\right)$ é uma submersão Riemanniana linear. Considere a translação desta submersão Riemanniana por $W_{p}$, que é a submersão Finsleriana linear $d \pi_{p}:\left(T_{p} M, Z_{p}\right) \rightarrow\left(T_{\pi(p)} N, R_{\pi(p)}\right)$, onde $R_{\pi(p)}$ é a métrica Randers com data $\left(g, d \pi W_{p}\right)$. Por unicidade $F_{\pi(p)}=R_{\pi(p)}$. Como $\pi:(M, Z) \rightarrow(N, F)$ é uma submersão Finsleriana, $W$ é projetável e $g_{d \pi_{p}}$ define uma métrica Riemanniana em $N$ pelo levantamento horizontal. De fato dados $p, q \in \pi^{-1}(x)$ segue que $d \pi_{p}\left(B_{p}^{Z}(1)\right)=d \pi_{p}\left(B_{q}^{Z}(1)\right)=B_{x}^{R}(1)$, $\operatorname{logo}$ $d \pi_{p} W=d \pi_{q} W$ e $d \pi^{*} g_{\pi(p)}=d \pi^{*} g_{\pi(q)}$. E portanto $\pi:(M, h) \rightarrow(B, g)$ é a translação de uma submersão Riemanniana.

Corolário 3.1.1. Uma folheação regular $\mathcal{F}$ é Randers com data $(h, W)$ se, e somente se, $W$ é projetável com $h(W, W)<1$ e $(M, h, \mathcal{F})$ é uma folheação Riemanniana

Proposição 3.1.1. Uma folheação singular sobre uma variedade Randers $(M, Z)$ com data $(h, W)$ é Z-homogênea, se e somente se, for h-homogênea e $W$ folheado.

A demonstração desta última Proposição é imediata a partir do Lema abaixo.

Lema 3.1.1 ([9], Lema 1.2). Seja $\phi:\left(M_{1}, R_{1}\right) \rightarrow\left(M_{2}, R_{2}\right)$ um difeomorfismo, onde $R_{i}=$ $\sqrt{a_{i}}+\beta_{i}$ é a métrica de Zermelo com data $\left(h_{i}, W_{i}\right)$, para qualquer $i \in(1,2)$. Então as proposições abaixo são equivalentes:

i. $\phi^{*} R_{2}=R_{1}$.

ii. $\phi^{*} h_{2}=h_{1}$ e d $\phi W_{1}=W_{2}$.

iii. $\phi^{*} a_{2}=a_{1}$ e $\phi^{*} \beta_{2}=\beta_{1}$.

Demonstração. $[i \Rightarrow i i]$ Abaixo será verificado que $R_{1}$ é métrica de Zermelo com data $\left(\phi^{*} h_{2}, d \phi^{-1} W_{2}\right)$. Isto permite concluir o item ii por unicidade da data, pois $R_{1}$ também é métrica de Zermelo com data $\left(h_{1}, W_{1}\right)$. Dado $v \in \mathcal{I}_{p}^{R_{1}}$, pelo item i, $R_{1}(v)=R_{2}(d \phi v)=1$. Como $R_{2}$ é métrica de Zermelo com data $\left(h_{2}, W_{2}\right)$ temos que

$$
1=h_{2}\left(d \phi v-W_{2}\right)=\left(\phi^{*} h_{2}\right)\left(v-d \phi^{-1} W_{2}\right),
$$

implicando a afirmação inicial. Já as implicações $[i i \Rightarrow i i i \Rightarrow i]$ seguem por cálculos explícitos.

Proposição 3.1.2. Seja $(M, \mathcal{F})$ uma folheação singular. Considere $R$ uma métrica Randers em $M$ com data $(h, W)$. Suponha que $W$ é um campo infinitesimalmente homotético e folheado. Então $(M, R, \mathcal{F})$ é uma folheação Finsleriana singular se, e somente se, $(M, h, \mathcal{F})$ é folheação Riemanniana singular.

Demonstração. Provaremos que uma geodésica de $h$ é $h$-horizontal se, e somente se, a geodésica unitária de $R$ associada também é $R$-horizontal (está no cone horizontal as folhas).

Sejam $\rho$ e $\gamma$ geodésicas de $(M, h)$ e $(M, R)$ dadas no Teorema 1.9.2. Observe que $\gamma^{\prime}(t)=$ $W_{\gamma(t)}+d \varphi_{t}\left(\rho^{\prime}(t)\right)$. Pelos Lemas 1.7.1 e 1.9.1

$$
\begin{aligned}
g_{\gamma^{\prime}(t)}\left(\gamma^{\prime}(t), u\right) & =\frac{R\left(\gamma^{\prime}(t)\right)}{\lambda \alpha\left(\gamma^{\prime}(t)\right)} h\left(\gamma^{\prime}(t)-R\left(\gamma^{\prime}(t)\right) W, u\right) \\
& =\frac{R\left(\gamma^{\prime}(t)\right)}{\lambda \alpha\left(\gamma^{\prime}(t)\right)} h\left(d \varphi_{t}\left(\rho^{\prime}(t)\right)+\left(1-R\left(\gamma^{\prime}(t)\right) W, u\right)\right. \\
& =\frac{1}{\lambda \alpha\left(\gamma^{\prime}(t)\right)} \varphi_{t}^{*} h\left(\rho^{\prime}(t),\left(d \varphi_{t}\right)^{-1} u\right),
\end{aligned}
$$

pois $\gamma$ é geodésica unitária de $R$. Portanto $\gamma^{\prime}(t)$ está no cone horizontal se, e somente se, $\rho^{\prime}(t)$ é horizontal com respeito a $h$, pois por suposição $d \varphi_{t}\left(T_{p} L_{p}\right)=T_{\varphi_{t}(p)} L_{\varphi(p)}$. 


\subsection{Exemplos Não-Homogêneos.}

Nesta seção, usando o resultado de Radeschi [23], serão construídos infinitos exemplos de folheações Randers singulares não-homogêneas.

Um sistema de Clifford é uma família $C=\left\{P_{0}, P_{1}, \ldots, P_{m}\right\}$ de matrizes simétricas em $\left(\mathbb{R}^{2 l},\langle\rangle,\right)$ tais que $P_{i}^{2}=I d$ para todo $i \in\{0, \ldots, m\}$ e $P_{i} P_{j}=-P_{j} P_{i}$ para $i \neq j$. A um sistema de Clifford $C$ associamos a aplicação $\pi_{C}: S^{2 l-1} \rightarrow \mathbb{R}^{m+1}$ definida por $\pi_{C}(x)=$ $\left(\left\langle P_{0} x, x\right\rangle, \ldots,\left\langle P_{m} x, x\right\rangle\right)$, que é denominada submersão de Clifford.

Teorema 3.2.1 (Radeschi). Seja $C=\left\{P_{0}, \ldots, P_{m}\right\}$ um sistema de Clifford em $\mathbb{R}^{2 l}$. Então a imagem de $\pi_{C}$ está contida em $\mathbb{D}_{C}$ disco centrado na origem em $\mathbb{R}^{m+1}$. Além disso, temos que

1. As fibras de $\pi_{C}$ são conexas se, e somente se, $l \neq m+1$ e neste caso define uma folheação Riemanniana singular em $\left(S^{2 l-1}, g, \mathcal{F}_{C}\right)$ cujo espaço de folhas ou é $\mathbb{S}_{C}=\partial \mathbb{D}_{C}$ $($ se $l=m)$ ou o disco $\mathbb{D}_{C}($ se $l>m+1)$. Em qualquer um dos casos a métrica induzida no quociente é a métrica redonda de curvatura 4.

2. A folheação $\left(\mathbb{S}^{2 l-1}, \mathcal{F}_{C}\right)$ é homogênea se, e somente se, $m=1,2$ ou $m=4$ e $P_{0} \cdot P_{1} \cdot P_{2} \cdot P_{3} \cdot P_{4}$ é $I d$ ou $-I d$.

Dado um sistema de Clifford $C=\left\{P_{0}, \ldots, P_{m}\right\}$ em $\mathbb{R}^{2 l}$ com $l>m+1$ e $m \neq 1,2,4$, seja $\left(S^{2 l-1}, g, \mathcal{F}_{C}\right)$ a folheação Riemanniana singular dada pelas fibras de $\pi_{C}$. Neste caso, os estratos singulares de $\mathcal{F}_{C}$ são as pré-imagens de 0 e de $\mathbb{S}_{C}$. Seja $\langle$,$\rangle métrica Riemanniana em$ $\operatorname{int}\left(\mathbb{D}_{C}\right)$ com curvatura 4 . Verifica-se que $\left.\pi_{C}\right|_{M_{0}}:\left(M_{0}, g\right) \rightarrow\left(\operatorname{int}\left(\mathbb{D}_{C}\right),\langle\rangle,\right)$ é uma submersão Riemanniana, onde $M_{0}$ é o conjunto dos pontos regulares. Considere $\tilde{W}$ um campo em $\mathbb{R}^{m+1}$ que se anula em uma vizinhança da origem e em outra vizinhança de $\mathbb{S}_{C}$ e também $\langle\tilde{W}, \tilde{W}\rangle<1$. Seja $W$ campo em $S^{2 l-1}$ tal que $d \pi_{C} W=\tilde{W}$ que por construção é um campo projetável tangente aos estratos. Observe que podemos escolher $W$ tal que $g(W, W)<1$. Portanto $\mathcal{F}_{C}$ é uma folheação Randers singular em $S^{2 l-1}$ com data $(g, W)$.

Pelo Lema 3.1.1, se $\mathcal{F}_{C}$ não é $g$-homogêneo, então $\mathcal{F}_{C}$ também não é $Z$-homogêneo. E como existem infinitas folheações Riemannianas singulares não-homogêneas geradas por sistemas de Clifford (Teorema 3.2.1 (b)) segue o corolário abaixo:

Corolário 3.2.1. Existe uma quantidade infinita de folheações Randers singulares nãohomogêneas na esfera.

\subsection{Folheação Finsleriana singular em espaços Randers- Minkowski}

Os espaços Randers-Minkowski são os modelos infinitesimais das variedades Randers. Verifica-se que um espaço Randers-Minkowski é um espaço Randers com data $(h, W)$ tal que $h$ é uma métrica Euclidiana (modelo infinitesimal das variedades Riemannianas) e $W$ é um campo constante. Compreender as folheações Finslerianas singulares com uma folha 0dimensional neste tipo de espaço é um passo fundamental para a demonstração do Teorema 1.

Lema 3.3.1. Seja $\left(\mathbb{R}^{n}, Z, \mathcal{F}\right)$ folheação Finsleriana singular sobre um espaço RandersMinkowski $\left(\mathbb{R}^{n}, Z\right)$ com data $(h, W)$ tal que $L_{0}=\{0\}$. Então $h(W(v), x)=0$ para qualquer $x \in T_{v} L$. Em particular, $\mathcal{F}$ é subfolheação da folheação $\mathcal{F}^{W}$ dada para cada $v \in V$ pelo hiperplano $V(v)$ paralelo a $W^{\perp}:=\left\{x \in \mathbb{R}^{n} ;\langle x, W\rangle=0\right\}$. 
Demonstração. Como $\mathcal{F}$ é folheação Finsleriana singular e a origem é uma folha, $v$ e $-v$ são ortogonais a $L_{v}$. De fato, basta notar que as geodésicas $\gamma(t)=v+t v$ e $\tilde{\gamma}(t)=v+(1-t) v$, que realizam respectivamente $v$ e $-v$, são ortogonais a origem.

Seja $x \in T_{v} L$. Se $v$ é a origem, não temos o que fazer. Se $v$ é não-nulo, pelo que discutimos acima $g_{v}(v, x)=g_{-v}(-v, x)=0$. Tais igualdades juntamente com o Lema 1.7.1 implicam que

$$
h(W, x)=\frac{h(x, v)}{Z(v)}=-\frac{h(x, v)}{Z(-v)} .
$$

E como $Z(v)$ e $Z(-v)$ são números positivos, podemos concluir que $h(W, x)=0$.

Proposição 3.3.1. Seja $\left(\mathbb{R}^{n}, Z\right)$ espaço Randers-Minkowski com data $(h, W)$. Se $\left(\mathbb{R}^{n}, Z, \mathcal{F}\right)$ é folheação Finsleriana singular cuja a folha minima é 0-dimensional, então $W$ é tangente ao estrato minimo.

Demonstração. Seja $\Sigma$ o estrato mínimo. Considere $v=t W$ para algum $t \in \mathbb{R}$. Para verificar a proposição basta provar que $v \in \Sigma$, ou equivalentemente, que $L_{v}=\{v\}$.

Pelo Lema 3.3.1, $L_{v} \subset V(v)$. Por outro lado, por 0 ser uma folha e pela estrutura tubular (Proposição 2.6.1), $L_{v} \subset \partial B_{\epsilon}^{+}(0)$. Porém como o espaço tangente a indicatriz $\partial B_{\epsilon}^{+}(0)$ $(\epsilon=Z(v))$ é o hiperplano afim $V(v)$ (veja o Exemplo 1.8.1) segue que $V(v) \cap \partial B_{\epsilon}^{+}(0)=\{v\}$, pela convexidade das indicatrizes, e portanto $L_{v}=\{v\}$.

Corolário 3.3.1. Seja $\mathcal{F}$ folheação Finsleriana singular sobre uma variedade RandersMinkowski $\left(\mathbb{R}^{n}, Z\right)$ com data $(h, W)$ cuja origem é uma componente conexa do estrato singular. Então $\left(\mathbb{R}^{n}, h, \mathcal{F}\right)$ é uma folheação Riemanniana singular.

\subsection{Prova do Teorema 1}

Suponha que $(M, Z, \mathcal{F})$ é uma folheação Finsleriana singular sobre uma variedade Randers com data $(h, W)$. Será verificado que $W$ é tangente aos estratos, olhando para a folheação infinitesimal, que é uma folheação Finsleriana singular em um espaço Randers-Minkowski. Uma vez que o vento seja tangente aos estratos $\left.\mathcal{F}\right|_{\Sigma}$, a restrição de $\mathcal{F}$ a um estrato $\Sigma=\Sigma_{k}$, é uma folheação Finsleriana regular em $\Sigma$ com a métrica Randers dada pela data $\left(\left.h\right|_{T \Sigma},\left.W\right|_{\Sigma}\right)$. Pelo Corolário 3.1.1, $\left.W\right|_{\Sigma}$ é projetável e $\left(\Sigma,\left.h\right|_{T \Sigma},\left.\mathcal{F}\right|_{\Sigma}\right)$ é folheação Riemanniana. E portanto $(M, h, \mathcal{F})$ é uma folheação Riemanniana singular pela Proposição 2.0.2, terminando a prova.

Proposição 3.4.1. Se $\mathcal{F}$ é folheação Finsleriana singular em uma variedade Randers $(M, Z)$ com data $(h, W)$, então $W$ é tangente aos estratos.

Demonstração. Dado $q \in M$, considere $\left(S, Z_{S}, \mathcal{F}_{q}\right)$ uma folheação infinitesimal por $q$ construida no Teorema de Slice 2.0.2. Pela Proposição 2.7.2, podemos considerar que $\left(S, Z_{S}, \mathcal{F}_{q}\right)$ é uma folheação Finsleriana singular em um espaço Randers-Minkowski com data $\left(\left.h\right|_{S}, W_{S}(q)\right)$ que possui $q$ como folha singular, onde $W_{S}(q)$ é a componente de $W(q)$ tangente a $S$. Pela Proposição 3.3.1, $W_{S}$ é tangente ao estrato mínimo de $\mathcal{F}_{q}$ e portanto o vetor $W(q)$ é tangente ao estrato que contém $q$, pois $T_{q} M$ coincide com $T_{q} L_{q}^{2} \oplus T_{q} S$ e $L_{q}^{2}$ é uma folha de $\mathcal{F}^{2}$ de $L_{q}$. 


\section{Capítulo 4}

\section{Submersão Finsleriana Singular}

O objetivo deste último capítulo é generalizar o artigo [2], mais especificamente, provar que as fibras de uma submersão Finsleriana singular analítica são equifocais.

Seja $\pi: M \rightarrow B$ uma aplicação suave e $M_{0}$ o conjunto dos pontos regulares. Se $\left.\pi\right|_{M_{0}}$ é uma submersão, então a aplicação $\pi$ é dita uma submersão singular. Adicionalmente se $\left.\pi\right|_{M_{0}}$ uma submersão Finsleriana, então $\pi$ será denominada submersão Finsleriana singular.

Uma fibra regular $L$ é dita equifocal quando para qualquer $\xi$ campo básico ao longo de $L$ (isto é, projetável e ortogonal a $L$ ) a aplicação end-point $\eta_{\xi}: L \rightarrow M$ definida por $\eta_{\xi}(p)=\gamma_{\xi}(p)$ tem posto constante. A aplicação $\pi$ é dita equifocal quando qualquer fibra regular é equifocal.

Teorema 4.0.1. Se $\pi:(M, F) \rightarrow B$ é uma submersão Finsleriana singular analítica com fibras conexas, então $\pi$ é equifocal.

Um exemplo de submersão Finsleriana singular é a submersão de Clifford $\pi_{C}$ dada por um sistema de Clifford $C=\left\{P_{0}, \ldots, P_{m}\right\}$ em $\mathbb{R}^{2 l}$ tal que $l>m+1$ construída na seção 3.2. Neste caso as fibras constituem uma folheação Finsleriana singular, sugerindo a conjectura de que tal conclusão pode ser verdadeira para qualquer submersão Finsleriana singular analítica.

Na primeira seção serão construídas as distribuições de Wilking para uma folheação singular, conceito usado na demonstração do principal resultado deste capítulo. Na segunda seção mostraremos o Teorema 4.0.1.

\subsection{Distribuições de Wilking}

No que se segue serão construídas as distribuições de Wilking associadas a uma folheação singular em uma variedade Finsleriana. Para isso primeiro serão definidos campos de Jacobi gerados a partir de uma variação por geodésicas ortogonais a uma subvariedade $P$, chamado de campos $P$-Jacobi, e explorado alguns resultados.

\subsubsection{Operador Forma e Campos P-Jacobi}

Sejam $P$ uma subvariedade de $M$ e $\pi: T M \rightarrow M$ a projeção canônica. Dado um vetor $v=v_{p} \in \pi^{-1}(P)$, considere o espaço euclidiano $\left(T_{p} M, g_{v}\right)$ e denote por $\tan ^{v}$ e nor $^{v}$ respectivamente as projeções ortogonais canônicas sobre $T_{p} P$ e $\operatorname{nor}^{v}(P):=\left\{u \in T_{p} M ; g_{v}\left(u, T_{p} P\right)=0\right\}$.

Dado um campo $N$ normal a $P$, definimos a segunda forma fundamental de $P$ na direção $N$ como a função

$$
\begin{aligned}
S_{N}^{P}: \Gamma(T P) \times \Gamma(T P) & \rightarrow \Gamma(N) \\
(U, W) & \mapsto \operatorname{nor}^{N}\left(\nabla_{U}^{N} W\right),
\end{aligned}
$$


E definimos o operador forma como

$$
\begin{aligned}
\tilde{S}_{N}^{P}: T P & \rightarrow T P \\
u & \mapsto-\tan ^{N}\left(\nabla_{u}^{N} N\right) .
\end{aligned}
$$

Quando não houver risco de confusão o índice $P$ em $S_{N}$ e $\tilde{S}_{N}$ será omitido.

Proposição 4.1.1. Com as notações acima, $S_{N}$ é $\mathcal{F}(P)$-bilinear e simétrica, enquanto $\tilde{S}_{N}$ é $\mathcal{F}(P)$-linear. Ademais,

$$
g_{N}\left(S_{N}(U, W), N\right)=g_{N}\left(\tilde{S}_{N}(U), W\right),
$$

para qualquer $U, W \in \Gamma(T P)$. Em particular, $g_{N}\left(\tilde{S}_{N}(U), W\right)=g_{N}\left(U, \tilde{S}_{N}(W)\right)$.

Demonstração. Por definição é imediato verificar que $\tilde{S}_{N}$ e $S_{W}(\cdot):=S_{N}(\cdot, W)$ são $\mathcal{F}(P)$ lineares para qualquer campo vetorial $W$ e que $S_{N}\left(, W_{1}+W_{1}\right)=S_{N}\left(, W_{1}\right)+S_{N}\left(, W_{2}\right)$. Para provar que $S_{N}(\cdot, f W)=f S_{N}(\cdot, W)$ para qualquer $f \in \mathcal{F}(P)$, basta notar que

$$
\operatorname{nor}^{N}\left(\nabla_{U}^{N} W\right)-\operatorname{nor}^{N}\left(\nabla_{W}^{N} U\right)=\operatorname{nor}^{N}([U, W])=0,
$$

pois $[U, W]$ é tangente a $P$. E por fim, pelo Lema 1.11 .1

$$
\begin{aligned}
g_{N}\left(S_{N}(U, W), N\right) & =g_{N}\left(\nabla_{U}^{N}(W), N\right)=U g_{N}(W, N)-g_{N}\left(W, \nabla_{U}^{N} N\right) \\
& =-g_{N}\left(W, \nabla_{U}^{N}(N)\right)=g_{N}\left(W, \tilde{S}_{N}(U)\right)
\end{aligned}
$$

pois $U$ é tangente a $P$.

Definição 4.1.1. Seja $\gamma:\left(t_{0}-\epsilon, t_{0}+\epsilon\right) \rightarrow M$ uma geodésica ortogonal a uma subvariedade $P$ de $M$ em $t_{0}$. Dizemos que um campo de Jacobi $J$ ao longo de $\gamma$ é P-Jacobi quando satisfizer as duas condições abaixo:

$$
J\left(t_{0}\right) \in T_{\gamma\left(t_{0}\right)} P \quad e \tan ^{\gamma^{\prime}\left(t_{0}\right)}\left(J^{\prime}\left(t_{0}\right)\right)+\tilde{S}_{\gamma^{\prime}}^{P}\left(J\left(t_{0}\right)\right)=0 .
$$

Diremos que um ponto $p \in M$ é P-focal quando existir algum campo P-Jacobi ao longo de $\gamma: \mathbb{R} \rightarrow M$ uma geodésica ortogonal a $P$ em $t_{0}$ tal que $p=\gamma(t)$ e $J(t)=0$ para algum $t \in \mathbb{R} \backslash\left\{t_{0}\right\}$.

A dimensão do subespaço formado por todos campos $P$-Jacobi ao longo de uma geodésica fixada é $n=\operatorname{dim} M$. Para verificar esta afirmação considere a aplicação

$$
(u, v) \in T_{\gamma\left(t_{0}\right)} P \times n \operatorname{nor}^{\gamma^{\prime}\left(t_{0}\right)}(P) \mapsto J,
$$

onde $J$ é o campo de Jacobi definido por $J\left(t_{0}\right)=u$ e $J^{\prime}\left(t_{0}\right)=\tilde{S}_{\gamma^{\prime}\left(t_{0}\right)}(u)+v$. Esta aplicação é linear, injetora e sua imagem coincide com o espaço dos campos $P$-Jacobi.

Lema 4.1.1. Seja J campo de Jacobi ao longo de $\gamma:\left(t_{0}-\epsilon, t_{0}+\epsilon\right) \rightarrow M$ geodésica ortogonal à subvariedade $P$ em $t_{0}$. Então $J$ é um campo P-Jacobi se, e somente se, for o campo variacional de uma variação por geodésicas ortogonais a $P$.

Demonstração. Seja $\gamma$ geodésica ortogonal a $P$ em $t_{0}$. Dada curva suave $N:(-\epsilon, \epsilon) \rightarrow \nu(P)$ com $N(0)=\gamma^{\prime}\left(t_{0}\right)$, considere a variação $H(t, s)=\exp _{\beta(s)} t N(s)$, onde $\beta(s):=\pi(N(s))$. Observe que esta é uma variação de $\gamma$ por geodésicas ortogonais a $P$. Denote por $V=V_{N}$ o campo variacional de $H$. Seja $(\Omega, \varphi)$ sistema de coordenadas em uma vizinhança de $\gamma\left(t_{0}\right)$, 
com $N^{i}, \beta^{i}$ e $\gamma^{i}(i=1, \ldots, n)$ as coordenadas de $N, \beta$ e $\gamma$, respectivamente, e $\Gamma_{i j}^{k}$ símbolos de Christoffel da conexão de Chern em $(\Omega, \varphi)$. Então

$$
D_{\gamma}^{\gamma^{\prime}} V\left(t_{0}\right)=D_{\beta}^{\gamma} N(0)=\left(\frac{d}{d s} N^{i}(0)+\left(\beta^{j}\right)^{\prime}(0)\left(\gamma^{k}\right)^{\prime}\left(t_{0}\right) \Gamma_{j k}^{i}\left(\gamma^{\prime}\left(t_{0}\right)\right) \partial_{i} .\right.
$$

Em particular, $\tan ^{\gamma^{\prime}}\left(D_{\gamma}^{\gamma^{\prime}} V\left(t_{0}\right)\right)=\tan ^{\gamma^{\prime}}\left(D_{\beta}^{\gamma^{\prime}} N(0)\right)=-\tilde{S}_{\gamma}\left(\beta^{\prime}(0)\right)=-\tilde{S}_{\gamma}(J(0))$ e portanto $V$ é um campo $L$-Jacobi.

Agora considere a aplicação $\eta: T_{\gamma^{\prime}} \nu(T L) \rightarrow T_{\gamma\left(t_{0}\right)} M \times T_{\gamma\left(t_{0}\right)} M$ definida por

$$
\eta\left(\beta^{\prime}, \frac{d}{d s} N\right)=\left(V\left(t_{0}\right), D_{\gamma}^{\gamma^{\prime}} V\left(t_{0}\right)\right)
$$

onde $V$ é o campo variacional construído a partir de $(\beta, N)$ como acima. Observe que sua imagem está contida no espaço dos campos $L$-Jacobi. Além disso, esta aplicação é injetora. De fato, se $\eta\left(\beta_{1}, \frac{d}{d s} N_{1}\right)=\eta\left(\beta_{2}, \frac{d}{d s} N_{2}\right)$, imediatamente concluímos que $\beta_{1}^{\prime}(0)=V_{1}\left(t_{0}\right)=V_{2}\left(t_{0}\right)=$ $\beta_{2}^{\prime}(0)$, logo pela equação 4.3 e por $D_{\gamma}^{\gamma^{\prime}} V_{1}\left(t_{0}\right)=D_{\gamma}^{\gamma^{\prime}} V_{2}\left(t_{0}\right)$ segue que $\frac{d}{d s} N_{1}\left(t_{0}\right)=\frac{d}{d s} N_{2}\left(t_{0}\right)$. E por fim, como a dimensão do espaço dos campos $L$-Jacobi é igual a dimensão de $\nu(L)$, podemos concluir que a imagem de $\eta$ coincide com o espaço dos campos $L$-Jacobi, terminando a prova.

Lema 4.1.2. Sejam $\pi:(M, F) \rightarrow(B, Z)$ submersão Finsleriana, $\gamma$ uma geodésica horizontal e J um campo $L_{\gamma\left(t_{0}\right)}$-Jacobi ao longo de $\gamma$ definido pela variação por geodésicas horizontais $H(t, s)$, onde $L_{\gamma\left(t_{0}\right)}$ é a fibra de $\pi$ passando por $\gamma\left(t_{0}\right)$. Temos que $J$ é vertical (isto é, tangente as fibras) se, e somente se, $\gamma_{s}^{\prime}(0)=\left.\frac{d}{d t}\right|_{t=0} H(t, s)$ é básico.

Demonstração. O campo $\gamma_{s}^{\prime}(0)$ é básico se, e somente se, $\gamma_{s}$ é o levantamento horizontal de $\pi \circ \gamma$ partindo de $H(0, s)$ para qualquer $s$, ou equivalentemente, $J(t)=\left.\frac{d}{d s}\right|_{s=0} H(t, s)$ é tangente as fibras.

Corolário 4.1.1. Se $J_{1}$ e $J_{2}$ são dois campos P-Jacobi ao longo de $\gamma: \mathbb{R} \rightarrow M$, com $\gamma\left(t_{0}\right) \in P$, ent $\tilde{a} o$

$$
g_{\gamma^{\prime}(t)}\left(J_{1}^{\prime}(t), J_{2}(t)\right)=g_{\gamma^{\prime}(t)}\left(J_{1}(t), J_{2}^{\prime}(t)\right)
$$

para qualquer $t \in \mathbb{R}$.

Demonstração. Como $J_{1}\left(t_{0}\right)$ e $J_{2}\left(t_{0}\right)$ são tangentes a $P$, pelo Lema 4.1.1 e Proposição 4.1.1, segue que

$$
\begin{aligned}
g_{\gamma^{\prime}\left(t_{0}\right)}\left(J_{1}\left(t_{0}\right), J_{2}^{\prime}(0)\right) & =g_{\gamma^{\prime}\left(t_{0}\right)}\left(J_{1}\left(t_{0}\right), \tilde{S}_{\gamma^{\prime}\left(t_{0}\right)}\left(J_{2}\left(t_{0}\right)\right)\right) \\
& =g_{\gamma\left(t_{0}\right)}\left(\tilde{S}_{\gamma^{\prime}\left(t_{0}\right)}\left(J_{1}\left(t_{0}\right)\right), J_{2}\left(t_{0}\right)\right)=g_{\gamma^{\prime}\left(t_{0}\right)}\left(J_{1}^{\prime}\left(t_{0}\right), J_{2}\left(t_{0}\right)\right),
\end{aligned}
$$

e portanto, pela Proposição 1.12.1, $g_{\gamma^{\prime}(t)}\left(J_{1}(t), J_{2}^{\prime}(t)\right)=g_{\gamma^{\prime}(t)}\left(J_{1}(t), J_{2}^{\prime}(t)\right)$, para qualquer $t \in(a-\epsilon, a+\epsilon)$.

\subsubsection{Distribuições de Wilking}

Seja $W$ o subespaço $(n-1)$-dimensional do espaço dos campos de Jacobi ao longo de uma geodésica $\gamma$ em $M^{n}$ que são $g_{\gamma^{\prime}}$-ortogonais a $\gamma^{\prime}$. Dizemos que $W$ é um subespaço auto-adjunto quando

$$
g_{\gamma^{\prime}(t)}\left(D_{\gamma(t)}^{\gamma^{\prime}} J_{1}, J_{2}(t)\right)=g_{\gamma^{\prime}(t)}\left(J_{1}(t), D_{\gamma(t)}^{\gamma^{\prime}} J_{2}\right)
$$


$J_{1}, J_{2} \in W$ e $t \in \mathbb{R}$. Pela Proposição 1.12.1, para mostrar que $W$ é auto-adjunto basta mostrar a equação 4.5 em um instante $t_{0}$.

Agora considere $V$ um subespaço próprio de $W$, em particular, $V$ também é auto-adjunto. Defina para qualquer instante $t$ os seguintes subespaços de $T_{\gamma(t)} M$ :

$$
V(t):=V_{1}(t) \oplus V_{2}(t)
$$

onde $V_{1}(t)=\{J(t) ; J \in V\}$ e $V_{2}(t)=\left\{J^{\prime}(t) ; J \in V\right.$ e $\left.J(t)=0\right\}$; e

$$
H(t):=V(t)^{\perp}:=\left\{u \in T_{\gamma(t)} M ; g_{\gamma^{\prime}(t)}(v, u)=0 \forall v \in V(t)\right\}
$$

isto é, complemento ortogonal de $V(t)$ com respeito a métrica Riemanniana $g_{\gamma^{\prime}}$ no fibrado normal à geodésica $\gamma$. Denote por $(\cdot)^{v}$ e $(\cdot)^{h}$ as projeções ortogonais com respeito à métrica $g_{\gamma^{\prime}}$ sobre $V(t)$ e $H(t)$ respectivamente. Desta forma qualquer vetor $u \in \gamma^{\prime}(t)^{\perp}:=\{u \in$ $\left.T_{\gamma(t)} M ; g_{\gamma^{\prime}}\left(\gamma^{\prime}, u\right)=0\right\}$ pode ser escrito de forma única como $u=u^{v}+u^{h}$, onde $u^{v} \in V(t)$ e $u^{h} \in H(t)$.

Observação 4.2. Se $W$ é um espaço auto-adjunto e $Y$ é um campo geodésico definido em um aberto $U$ tal que $Y(\gamma(t))=\gamma^{\prime}(t)$, então $W$ é um espaço auto-adjunto da variedade Riemanniana $\left(U, g_{Y}\right)$.

Lema 4.2.1. Sejam $W$ um espaço auto-adjunto ao longo de uma geodésica $\gamma$ e $V$ um subespaço de $W$. Então

1. $\operatorname{dim} V=\operatorname{dim} V(t)$, para qualquer $t \in \mathbb{R}$.

2. O subconjunto $\left\{t \in \mathbb{R} ; V_{2}(t) \neq 0\right\} \subset \mathbb{R}$ é discreto.

3. Se $J \in W$, então a projeção $Y=J^{h}$ satisfaz a equação de Jacobi transversal, isto é,

$$
D_{t}^{h} D_{t}^{h} Y+\left(R_{\gamma^{\prime}} Y\right)^{h}+3 A_{t} A_{t}^{*} Y=0,
$$

onde $D^{h}$ é a conexão induzida no fibrado $H$, mais precisamente, $D^{h} X=\left(X^{\prime}\right)^{h}=$ $\left(D_{\gamma}^{\gamma^{\prime}} X\right)^{h}$, para qualquer seção $X \in \Gamma(H)$; e $A_{t}(X(t)):=\left(\left(X^{h}\right)^{\prime}(t)\right)^{v}+\left(\left(X^{v}\right)^{\prime}(t)\right)^{h}$ é o tensor de O'Neill para qualquer $X$ campo suave ao longo de $\gamma$.

Demonstração. Seja $\left.\gamma\right|_{I}$ segmento de $\gamma$ sem pontos conjugados. Em uma vizinhança de $\left.\gamma\right|_{I}$ podemos estender $\gamma^{\prime}$ para um campo geodésico $V$. Pela Proposicão 1.11.1 e pelo Lema 1.10.3, $W$ é um espaço auto-adjunto Riemanniano com respeito a métrica $\tilde{g}:=g_{V}$.

E como este lema é válido para o caso Riemanniano (vide [13]), segue que é verdadeiro para $\left.\gamma\right|_{I}$ no caso Finsler. Mais precisamente o item (3) segue adicionando a seguinte observação: pelo Lema $1.10 .3, \tilde{D}_{\gamma}=D_{\gamma}^{V}, \tilde{A}=A$ e $\tilde{R}=R_{\gamma^{\prime}}$, onde $\tilde{D}$, $\tilde{A}$ e $\tilde{R}$ são respectivamente a derivada covariante, o tensor de O'neill e a curvatura seccional com respeito a métrica $\tilde{g}$.

Por fim como os pontos conjugados são isolados, terminamos a demonstração cobrindo $\mathbb{R}$ por intervalos $I$ que não contêm pontos conjugados.

Considere $L$ uma folha regular de folheação singular $\mathcal{F}$ que possua uma vizinhança saturada $U$ de $L$ tal que $\left.\mathcal{F}\right|_{U}$, a restrição de $\mathcal{F}$ a $U$, é dada por uma submersão Finsleriana $\pi: U \rightarrow B$ e $\gamma: \mathbb{R} \rightarrow M$ geodésica ortogonal a $L$ em $t_{0}$ tal que os pontos singulares em $\gamma$ são isolados. Por exemplo, $\mathcal{F}$ uma folheação Finsleriana singular. Seja $W$ espaço dos campos $L$-Jacobi $g_{\gamma^{\prime}}$-ortogonais a $\gamma^{\prime}$, que é um auto-espaço $(n-1)$-dimensional. Defina $V$ subespaço formado pelos campos tipo holonomia ao longo de $\gamma$, isto é, campos $L$-Jacobi gerados pelos levantamentos horizontais de $\gamma$, que é um subespaço próprio de $W$. As distribuições $t \mapsto V(t)$ e $t \mapsto H(t)$ ao longo de $\gamma$ definidas respectivamente pelas equações 4.6 e 4.7 são denominadas distribuições de Wilking. 
Lema 4.2.2. Sejam $V$ e $H$ distribuições de Wilking associadas a folha $L=L_{\gamma\left(t_{0}\right)}$ da folheação Finsleriana singular $(M, F, \mathcal{F})$ sobre a geodésica horizontal $\gamma$. Se $\gamma(t)$ é regular, então $V(t)$ coincide com $T_{\gamma(t)} L_{\gamma(t)}$ e consequentemente $H(t) \oplus \gamma^{\prime}(t)$ coincide com o subespaço horizontal em $\gamma(t)$.

Demonstração. Dado $\gamma(t)$ ponto regular, seja $\pi: U \rightarrow B$ submersão Finsleriana que descreve $\mathcal{F}$ em $U$ vizinhança de $\gamma(t)$.

Os subespaços $V_{1}(t)$ e $T_{\gamma(t)} L_{\gamma(t)}$ coincidem para qualquer $t$ tal que $\gamma(t) \in U$. Dado $J \in V$ considere $H(t, s)$ campo variacional de $J$. As geodésicas $\gamma_{s}=H(, s)$ são levantamentos horizontais de $\pi \circ \gamma$, em particular, $\pi \circ \gamma(t)=\gamma_{s}(t)$, para quaisquer $t$ e $s$. Logo, para qualquer $t$, a curva $\beta_{t}:=H(t$,$) é uma curva contida em uma folha e portanto$

$$
J(t)=\left.\frac{\partial}{\partial s}\right|_{s=0} H(t, s)=\left.\frac{d}{d s}\right|_{s=0} \beta_{t}(s) \in T_{\gamma(t)} L_{\gamma(t)} .
$$

Reciprocamente, dado $v \in T_{\gamma(t)} L_{\gamma(t)}$, tome uma curva $\beta:[\epsilon, \epsilon] \rightarrow L_{\gamma(t)}$ tal que $\beta^{\prime}(0)=v$. Considere o campo de Jacobi $J$ gerado pela variação $H(t, s)$, onde $\gamma_{s}=H(, s)$ é o levantamento horizontal de $\pi \circ \gamma$ partindo de $\beta(s)$. Por definição, $v=J(t) \in V_{1}(t)$, pois por construção $J \in V$.

Por fim, verifiquemos que $V_{2}(t)=0$ para $\gamma(t)$ ponto regular. De fato, seja $J \in V$ com variação $H(t, s)$. Se $J(t)=0$, então $H(t, s)=\gamma(t)$ para todo $s$. Por definição para qualquer $s$ a geodésica $\gamma_{s}(t)=H(t, s)$ é o levantamento horizontal de $\pi(\gamma \cap U)$ passando por $\gamma_{s}(t)=\gamma(t)$. Logo $\gamma_{s}=\gamma$, implicando que $J^{\prime}=0$.

Proposição 4.2.1. Sejam $(M, F)$ uma variedade Finsleriana, $\pi:(U, F) \rightarrow(V, Z)$ uma submersão Finsleriana definida em algum aberto $U$ de $M, \gamma: \mathbb{R} \rightarrow M$ geodésica ortogonal a uma fibra $L$ em $t=0, W$ espaço auto-adjunto gerado pelos campos $L$-Jacobi $g_{\gamma^{\prime}}$-ortogonais $a \gamma^{\prime}$ e $V, H$ distribuições de Wilking associadas. Então para qualquer $t_{0} \in \mathbb{R}$ existe $\delta>0$ tal que se $J \in W$ e $s_{1}, s_{2} \in I:=\left(t_{0}-\delta, t_{0}+\delta\right)$ com $s_{1} \neq s_{2}$ e $J^{h}\left(s_{1}\right)=0=J^{h}\left(s_{2}\right)$ para todo $t$, então $J^{h}=0$.

Demonstração. Todo campo $Y=J^{h} \operatorname{com} J \in W$, satisfaz a equação de Jacobi transversal 4.8, que é um sistema Morse-Sturm. Por outro lado, pelo Lema 2.1 em [22], a equação Jacobi transversal é identificada com uma equação de Jacobi em uma variedade Riemanniana e neste caso para qualquer instante é possível escolher um intervalo que não contenha pontos conjugados. Isso termina a prova desta Proposição.

\subsection{Prova do Teorema 3}

Seja $\pi:(M, F) \rightarrow B$ uma aplicação analítica definida em uma variedade Finsleriana tal que $\mathcal{F}_{\pi}$, a restrição das fibras ao conjunto dos pontos regulares, é uma folheação Finsleriana. Fixe $L$ folha regular e $\xi$ um campo básico ao longo de $L$. Dado $x \in L$ denotaremos por $\gamma_{x}(t)=\eta_{t \xi}(x)$ a geodésica partindo de $x$ com velocidade $\xi(x)$.

Lema 4.3.1. Se $\gamma$ é uma geodésica que intersecta o conjunto regular, então as singularidades em $\gamma$ são isoladas.

Demonstração. Seja $k$ a dimensão da folheação, isto é, $k$ é a dimensão da folha regular. Suponha que $\gamma(0)$ é regular. Considere a família $\left\{A_{\alpha}(t)\right\}$, onde $A_{\alpha}(t)$ é uma submatriz $k \times k$ da matriz de $d \pi_{\gamma(t)}$ em um referencial $g_{\gamma^{\prime}}$-ortonormal associado a uma carta fixada. Defina a função analítica $f=\sum f_{\alpha}^{2}$, onde $f_{\alpha}(t)=\operatorname{det} A_{\alpha}(t)$. Note que $\gamma(t)$ é singular se, e somente se, $f(t)=0$. Como os zeros de uma função analítica não-nula são isolados e $f(0)$ é não-nulo, os pontos singulares ao longo de $\gamma$ são isolados. 
Lema 4.3.2. O conjunto $\eta_{\xi}(L)$ está contido em um conjunto de nível.

Demonstração. Dados $x, y \in L$, defina a função $g(t)=\left(\pi \circ \gamma_{x}-\pi \circ \gamma_{y}\right)(t)$, que é analítica. E como a folheação é descrita por uma submersão Finsleriana em torno de $L, g$ é identicamente nulo em um intervalo e portanto em toda reta, por analiticidade.

Lema 4.3.3. Se $\gamma$ é uma geodésica tal que $\gamma^{\prime}(0)$ é ortogonal a L, então $\gamma$ é horizontal.

Demonstração. Nesta demonstração serão utilizadas as definições e resultados sobre distribuições de Wilking da seção 4.1.2.

As fibras em uma vizinhança $U$ de $L$ são dadas por uma submersão Finsleriana. Considere a distribuição de Wilking $V_{U}$ ao longo de $\gamma_{x} \cap U$. Por existência e unicidade de campos de Jacobi dadas condições iniciais, podemos estender os campos em $V_{U}$ para $\mathbb{R}$. Seja $V$ o subespaço formado pelas extensões dos campos em $V_{U}$, que é um espaço auto-adjunto relativo a $\gamma_{x}$. Observe que $V(t)=V_{1}(t)$ para todo $t$ pertencente a um subconjunto $R$ aberto e denso de $\mathbb{R}$. O subespaço $V(t)$ possui dimensão constante igual à dimensão da pré-imagem de um valor regular. Pelo Lema 4.3.2, $V_{1}(t)$ está contido no espaço tangente às folhas. E assim $V_{1}(t)$ coincide com $T_{\gamma_{x}(t)} L_{\gamma_{x}(t)}$ para todo $t \in R$. Em particular, $\gamma^{\prime}(t)$ é ortogonal para todo $t \in R$ e $R$ coincide com o conjunto dos instantes $t$ tal que $\gamma(t)$ é regular. Pela definição de folheação singular e por continuidade, concluimos que $\gamma$ é horizontal. De fato, suponha que $t_{0} \in R^{c}$; dado $v \in T_{\gamma\left(t_{0}\right)} L_{\gamma}$ existe campo vetorial $X$ que é tangente as folhas ao longo de $\left.\gamma\right|_{\left(t_{0}-\epsilon, t_{0}+\epsilon\right)}$ para algum $\epsilon>0$ suficientemente pequeno e $X\left(t_{0}\right)=v$. Note que $f(s)=g_{\gamma^{\prime}(s)}\left(\gamma^{\prime}(s), X(s)\right)=0$ para todo $s \in R \cap\left(t_{0}-\epsilon, t_{0}+\epsilon\right)$. Portanto $f\left(t_{0}\right)=0$, por continuidade.

Observe que o lema anterior não implica que a folheação é uma folheação Finsleriana singular, pois não mostra o caso em que $\gamma(0)$ é um ponto singular.

Lema 4.3.4. Se d é um valor regular, então $\eta_{\xi}$ é um difeomorfismo.

Demonstração. Construiremos a aplicação inversa da aplicação end-point. Fixe uma geodésica $\gamma_{p}$. Como $d$ é valor regular, podemos estender $\gamma_{p}^{\prime}(1)$ para um campo básico $\tilde{\xi}$ na fibra $\pi^{-1}(d)$. Dado $y \in \pi^{-1}(d)$, seja $\tilde{\gamma}_{y}$ a geodésica com velocidade $\tilde{\xi}(y)$. Defina a aplicação end-point passado $\eta_{\tilde{\xi}}^{-}: \pi^{-1}(d) \rightarrow M$ por $\eta_{\tilde{\xi}}^{-}(y)=\tilde{\gamma}_{y}(-1)$. Usando um argumento análogo ao presente no Lema 4.3.2, concluímos que a imagem da aplicação end-point passado está contida em $L$. Esta é nossa candidata a inversa da aplicação end-point.

Como vale $\pi \circ \eta_{t \xi}(p)=\pi \circ \eta_{t \xi}(x)$, para quaisquer $x \in L$ e $t \in \mathbb{R}$, tem-se que $d \pi \tilde{\xi}\left(\eta_{\xi}(x)\right)=$ $d \pi \gamma_{x}^{\prime}(1)$. Assim por definição $\tilde{\xi}\left(\eta_{\xi}(x)\right)=\gamma_{x}^{\prime}(1)$. Logo $\tilde{\gamma}_{\gamma_{x}(1)}(t)=\gamma_{x}(1+t)$, implicando que a aplicação end-point passado é inversa à esquerda da aplicação end-point:

$$
\eta_{\tilde{\xi}}^{-} \circ \eta_{\xi}(x)=\tilde{\gamma}_{\gamma(1)}(-1)=\gamma_{x}(0)=x .
$$

Analogamente, $\eta_{\xi} \circ \eta_{\tilde{\xi}}^{-}=I d_{\pi^{-1}(d)}$, pois $d \pi_{x}(\xi)=d \pi_{x} \tilde{\gamma}_{y}^{\prime}(-1)$.

Observação 4.4. Considere as distribuições de Wilking partindo de $L_{p}$ ao longo de $\gamma_{p}$. Os campos $L_{p^{-}}$Jacobi são identificados com os campos $L_{\tilde{p}^{-}}$Jacobi. Logo as equações de Jacobi transversal começando em $L_{p}$ e $L_{\tilde{p}}$ coincidem. E portanto os campos da forma $J(t)=\tilde{J}^{h}(t-$ $s)$, com $\tilde{J}$ campo $L_{\tilde{p}^{-}} J a c o b i$, são soluções da equação de Jacobi transversal começando em $L_{p}$ ao longo de $\gamma_{p}$.

Lema 4.4.1. O posto de $\eta_{\xi}$ é localmente constante. 
Demonstração. Considere $W$ o espaço auto-adjunto definido pelos campos $L$-Jacobi $g_{\gamma^{\prime-}}$ ortogonais e $V$ e $H$ distribuições de Wilking. Fixado $p \in L$ mostraremos que existe $U$ vizinhança aberta de $p$ em $L$ tal que para qualquer $x \in U$ o posto de $\eta_{\xi}$ em $x$ é igual ao posto de $\eta_{\xi}$ em $p$. Sem perda de generalidade podemos considerar que $F(\xi)=1$ e $\eta_{r \xi}(L) \subset L_{d}$.

Considere a geodésica $\gamma(t)=\eta_{t \xi}(p)$ e $\delta>0$ dado na Proposição 4.2 .1 para $t_{0}=r$. Pelo Lema 4.3.4, podemos assumir que $\gamma(0) \in \gamma(r-\delta, r+\delta)$. Logo se $J \in W \operatorname{com} J(r)=0$, então $J \in V$, ou seja, $J$ é tipo holonomia, pela Proposição 4.2.1. E portanto $k e r d\left(\eta_{\xi}\right)_{p}$ é gerado por $\{J(0) ; J \in V$ e $J(r)=0\}$. Como para alguma vizinhança aberta $U$ de $p$ em $L$ as equações de Jacobi transversal ao longo de $\gamma$ e de $\gamma_{x}$ para qualquer $x \in U$, a conclusão do parágrafo anterior também é válida para $\gamma_{x}$ e o mesmo $\delta$.

Seja $k$ o posto de $\eta_{\xi}$ em $p$. Provemos que dim ker $d\left(\eta_{t \xi}\right)_{x}=k$, para qualquer $x \in U \subset L$. Como os pontos críticos são tangenciais, temos que

$$
m\left(\gamma_{x}\right)=\sum_{i} \operatorname{dim} \operatorname{ker}_{t_{i}(x) \xi}(x)
$$

onde $t_{i}(x)$ e $m\left(\gamma_{x}\right)$ denotam, respectivamente, a distância de $x$ a um ponto $L$-focal em $\gamma_{x}$ e o número de pontos focais em $\gamma_{x}$ contando com as multiplicidades. Por analiticidade, os pontos críticos de $\pi$ em $\gamma_{x}$ são isolados. Suponha sem perda de generalidade que $\gamma_{x}(1)$ é o único ponto crítico ao longo de $\gamma_{x}$. Como a aplicação end-point é um difeomorfismo entre conjuntos de níveis regulares, segue que

$$
m\left(\gamma_{x}\right)=\operatorname{dim} \operatorname{ker} \eta_{\xi}(x) .
$$

Observe que dim ker $d\left(\eta_{\xi}\right)$ não pode aumentar (a dimensão da imagem de $d\left(\eta_{\xi}\right)$ não diminui) em uma vizinhança de $p$ em $L$ suficientemente pequena, logo

$$
m\left(\gamma_{p}\right) \geq m\left(\gamma_{x}\right)
$$

Por outro lado, o Teorema do Índice de Morse caso Finsler implica a existência de uma vizinhança $U \subset L$ de $p$ tal que

$$
m\left(\gamma_{x}\right) \geq m\left(\gamma_{p}\right)
$$

para todo $x \in U$. As equações 4.10, 4.11 e 4.12 nos permitem concluir o resultado. 


\section{Referências Bibliográficas}

[1] Marcos Alexandrino and Dirk Töben, Equifocality of a singular riemannian foliation, Proceedings of the American Mathematical Society 136 (2008), no. 9, 3271-3280. 12, 37

[2] Marcos M Alexandrino, Integrable riemannian submersion with singularities, Geometriae Dedicata 108 (2004), no. 1, 141-152. 57

[3] _ Desingularization of singular riemannian foliation, Geometriae Dedicata 149 (2010), no. 1, 397-416. 12, 37

[4] Marcos M Alexandrino, Benigno O Alves, and Miguel Angel Javaloyes, On singular finsler foliation, arXiv preprint arXiv:1708.05457 (2017). 13

[5] Marcos M Alexandrino and Renato G Bettiol, Lie groups and geometric aspects of isometric actions, Springer, 2015. 12, 37

[6] Marcos M Alexandrino, Rafael Briquet, and Dirk Töben, Progress in the theory of singular riemannian foliations, Differential Geometry and its Applications 31 (2013), no. 2, 248-267. 37

[7] J Álvarez Paiva and C Durán, Isometric submersions of finsler manifolds, Proceedings of the American Mathematical Society 129 (2001), no. 8, 2409-2417. 15, 29, 30

[8] Benigno Alves and Miguel Angel Javaloyes, A note on the existence of tubular neighbourhoods on finsler manifolds and minimization of orthogonal geodesics to a submanifold, arXiv preprint arXiv:1710.01699 (2017). 13

[9] David Bao, Colleen Robles, Zhongmin Shen, et al., Zermelo navigation on riemannian manifolds, Journal of Differential Geometry 66 (2004), no. 3, 377-435. 15, 54

[10] Erasmo Caponio, Miguel Ángel Javaloyes, and Antonio Masiello, On the energy functional on finsler manifolds and applications to stationary spacetimes, Mathematische Annalen 351 (2011), no. 2, 365-392. 22

[11] Erasmo Caponio, Miguel Angel Javaloyes, Miguel Sánchez, et al., On the interplay between lorentzian causality and finsler metrics of randers type, Revista Matemática Iberoamericana 27 (2011), no. 3, 919-952. 22

[12] GW Gibbons and CM Warnick, The geometry of sound rays in a wind, Contemporary Physics 52 (2011), no. 3, 197-209. 18

[13] Detlef Gromoll and Gerard Walschap, Metric foliations and curvature, vol. 268, Springer Science \& Business Media, 2009. 13, 60 
[14] Qun He, Songting Yin, and Yibing Shen, Isoparametric hypersurfaces in minkowski spaces, Differential Geometry and its Applications 47 (2016), 133-158. 15, 33, 35

[15] Miguel Angel Javaloyes, Chern connection of a pseudo-Finsler metric as a family of affine connections, Publ. Math. Debrecen 84 (2014), no. 1-2, 29-43. MR 3194771 15, $24,26,44$

[16] Miguel Ángel Javaloyes, Anisotropic tensor calculus, arXiv preprint arXiv:1602.05492 (2016). 43

[17] Miguel Angel Javaloyes and Miguel Sánchez, On the definition and examples of Finsler metrics, Ann. Sc. Norm. Super. Pisa Cl. Sci. (5) 13 (2014), no. 3, 813-858. MR 3331530 $15,16,18,22$

[18] Małgorzata Józefowicz and Robert Wolak, Finslerian foliations of compact manifolds are riemannian, Differential Geometry and its Applications 26 (2008), no. 2, 224-226. 11,38

[19] Serge Lang, Introduction to differentiable manifolds, Springer Science \& Business Media, 2006. 12, 15, 26, 27

[20] Steen Markvorsen, A finsler geodesic spray paradigm for wildfire spread modelling, Nonlinear Analysis: Real World Applications 28 (2016), 208-228. 18

[21] Pierre Molino and Grant Cairns, Riemannian foliations, Birkhauser Boston Inc., 1988. 12,37

[22] Paolo Piccione and Daniel V. Tausk, On the distribution of conjugate points along semiRiemannian geodesics, Comm. Anal. Geom. 11 (2003), no. 1, 33-48. MR 2016195 29, 61

[23] Marco Radeschi, Clifford algebras and new singular riemannian foliations in spheres, Geometric and Functional Analysis 24 (2014), no. 5, 1660-1682. 55

[24] Colleen Robles, Geodesics in randers spaces of constant curvature, Transactions of the American Mathematical Society (2007), 1633-1651. 21

[25] Zhongmin Shen, Lectures on finsler geometry, World Scientific, 2001. 15, 16, 26, 35

[26] Chuu-Lian Terng, Gudlaugur Thorbergsson, et al., Submanifold geometry in symmetric spaces, J. Differential Geom 42 (1995), no. 3, 665-718. 12

[27] Qi-Ming Wang, Isoparametric functions on riemannian manifolds. i, Mathematische Annalen 277 (1987), no. 4, 639-646. 33, 35 